This is a self-archived version of an original article. This version may differ from the original in pagination and typographic details.

Author(s): Campbell, P.; Moore, lain; Pearson, M.R.

Title: Laser spectroscopy for nuclear structure physics

Year: 2016

Version: Accepted version (Final draft)

Copyright: @ $\odot 2015$ Elsevier B.V. All rights reserved.

Rights: CC BY-NC-ND 4.0

Rights url: https://creativecommons.org/licenses/by-nc-nd/4.0/

Please cite the original version:

Campbell, P., Moore, I., \& Pearson, M.R. (2016). Laser spectroscopy for nuclear structure physics. Progress in Particle and Nuclear Physics, 86, 127-180.

https://doi.org/10.1016/j.ppnp.2015.09.003 


\section{Accepted Manuscript}

Laser spectroscopy for nuclear structure physics

P. Campbell, I.D. Moore, M.R. Pearson

PII:

S0146-6410(15)00091-5

DOI: $\quad$ http://dx.doi.org/10.1016/j.ppnp.2015.09.003

Reference: JPPNP 3596

To appear in: Progress in Particle and Nuclear Physics

Please cite this article as: P. Campbell, I.D. Moore, M.R. Pearson, Laser spectroscopy for nuclear structure physics, Progress in Particle and Nuclear Physics (2015),

http://dx.doi.org/10.1016/j.ppnp.2015.09.003

This is a PDF file of an unedited manuscript that has been accepted for publication. As a service to our customers we are providing this early version of the manuscript. The manuscript will undergo copyediting, typesetting, and review of the resulting proof before it is published in its final form. Please note that during the production process errors may be discovered which could affect the content, and all legal disclaimers that apply to the journal pertain. 


\title{
Laser spectroscopy for nuclear structure physics
}

\author{
P. Campbell, ${ }^{1}$ I.D. Moore,${ }^{2}$ M.R. Pearson,${ }^{3}$ \\ ${ }^{1}$ School of Physics and Astronomy, University of Manchester, \\ Oxford Road, Manchester, M13 9PL, UK \\ ${ }^{2}$ Department of Physics, University of Jyväskylä, \\ Survontie 9, Jyväskylä, FI-40014, Finland \\ ${ }^{3}$ TriumF, 4004 Westbrook Mall, Vancouver BC, V6T 2A3, Canada
}

September 21, 2015

\begin{abstract}
High-resolution laser spectroscopy is an established powerful tool in the study of nuclear shape, size and multipole moments. Measurements of the hyperfine structures and isotope shifts in the atomic spectra of radioactive nuclei provide unique insight into the evolution of the nuclear macroscopic shape and microscopic structure. These measurements can be made with high precision and high sensitivity and applied directly on-line at radioactive nuclear beam facilities. Recent measurements, advances at facilities and the future direction of the field are reviewed. A summary of experimental data is presented.
\end{abstract}




\section{Contents}

1 Introduction $\quad 4$

2 Nuclear perturbations of the atomic structure $\quad 9$

2.1 Nuclear ground state properties from atomic spectra . . . . . . . . . . . . . 9

2.2 Hyperfine structure . . . . . . . . . . . . . . . . . . . . . . . . . . 9

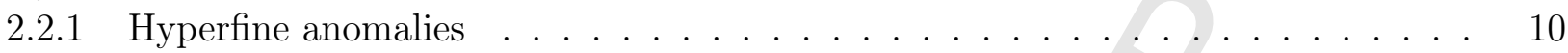

2.3 Isotope shift . . . . . . . . . . . . . . . . . . . . . . . 11

2.4 Mean-squared nuclear charge radii . . . . . . . . . . . . . . . . . . . . 12

2.5 Relation to other nuclear observables . . . . . . . . . . . . . . . . . . 13

3 Atomic Spectroscopy $\quad 15$

3.1 Excitation and detection of atomic transitions . . . . . . . . . . . . . . . . 15

3.2 Pioneering experiments . . . . . . . . . . . . . . . . . . . . 16

3.3 Sample preparation . . . . . . . . . . . . . . . . . . . . . . . . . 16

3.4 Collinear Laser Spectroscopy . . . . . . . . . . . . . . . . . . . . . . . . . 18

3.4.1 Precision collinear spectroscopy . . . . . . . . . . . . . . . . . . 19

3.4.2 Coincidence detection . . . . . . . . . . . . . . . . . . . . . 20

3.4 .3 Bunched beam spectroscopy . . . . . . . . . . . . . . . . . 20

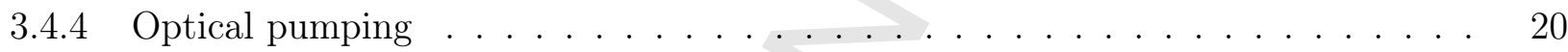

3.5 Resonance ionisation spectroscopy . . . . . . . . . . . . . . . . . 20

3.5.1 Hot cavity in-source resonance ionisation spectroscopy _ . . . . . . . . . . . . 24

3.5.2 Collinear resonance ionisation spectroscopy . . . . . . . . . . . . . . . . 26

3.6 Atom and ion traps . . . . . . . . . . . . . . . . . . . . . 27

3.6.1 Magneto-optical atom traps . . . . . . . . . . . . . . . . 27

3.6 .2 Ion traps . . . . . . . . . . . . . . . . . . . . . . . . . . . 29

3.7 Gas cells and ion guides . . . . . . . . . . . . . . . . . . . . 30

3.7.1 In-gas-cell resonance ionisation spectroscopy . . . . . . . . . . . . . . . . . 31

3.7.2 In-gas-jet resonance ionisation spectroscopy _ . . . . . . . . . . . . . . . . 33

3.8 Comparison of techniques . . . . . . . . . . . . . . . . . . . . . 34

4 On-line laser spectroscopy facilities $\quad 39$

4.1 Present and planned facilities . . . . . . . . . . . . . . . . . . . . . . 39

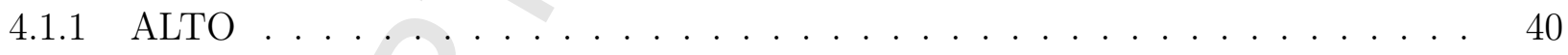

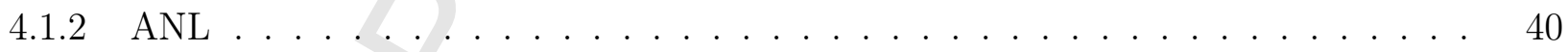

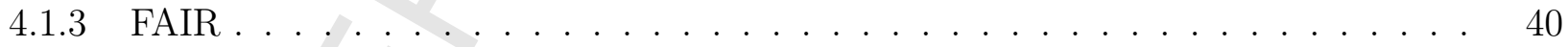

4.1 .4 GALS, DUBNA . . . . . . . . . . . . . . . . . 40

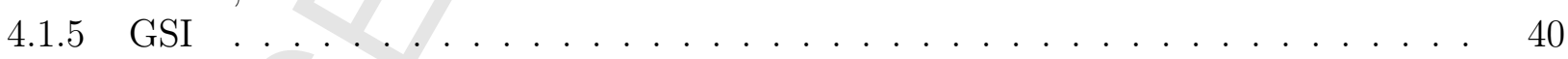

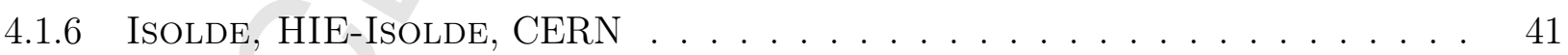

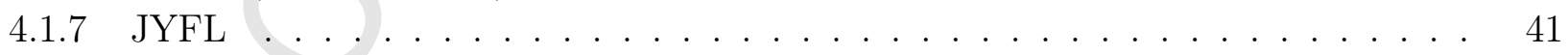

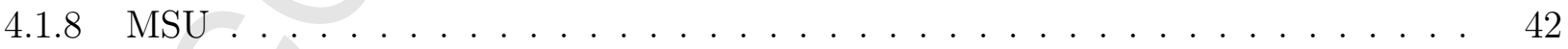

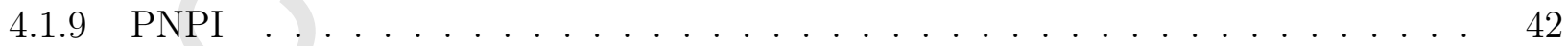

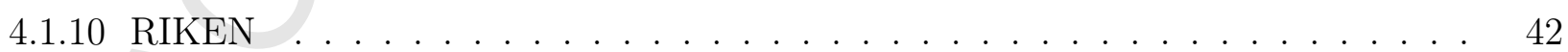

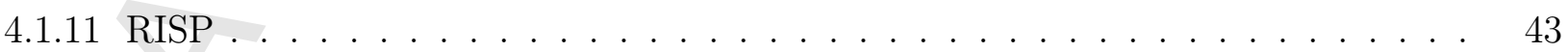

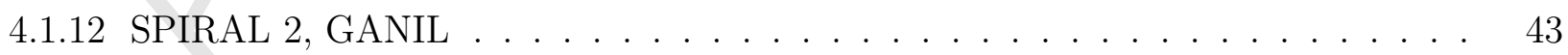

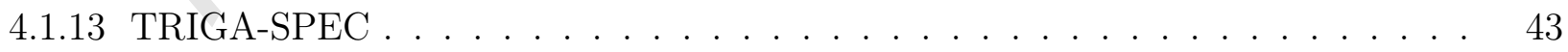

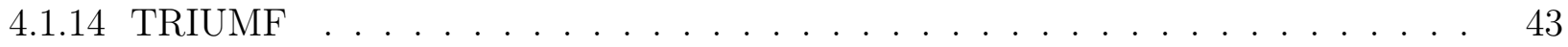

4.2 Recent changes . . . . . . . . . . . . . . . . . . . . . . . . . . . 44 
5 Light Mass nuclei $\quad 45$

5.1 Results and recent progress . . . . . . . . . . . . . . . . . . 45

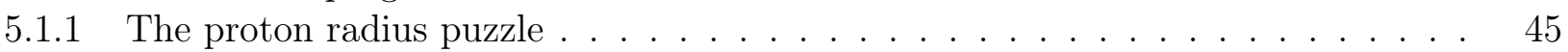

5.1 .2 Halo Nuclei $(\mathrm{He}, \mathrm{Li}, \mathrm{Be}) \ldots \ldots \ldots$. . . . . . . . . . . . . . . . . . . . . . . . . . 46

5.1 .3 From proton halos to beyond the Island of Inversion (Ne to Ti) $\quad \ldots . \ldots$

6 Medium Mass nuclei $\quad 51$

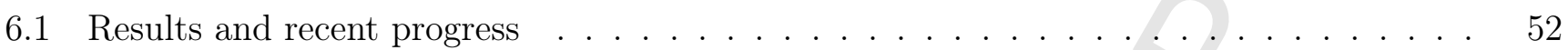

6.1 .1 Neutron-rich nuclei near $Z=28 \ldots \ldots \ldots \ldots \ldots \ldots$

6.1 .2 The zirconium region . . . . . . . . . . . . . . . . . . 54

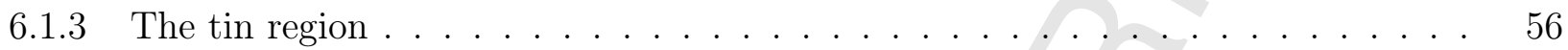

6.1.4 The mid-shell $(N \sim 104)$ platinum to lead nuclei . . . . . . . . . . . . 58

7 Heavy Mass nuclei $\quad 58$

7.1 Results and recent progress . . . . . . . . . . . . . . . . . . . . 59

7.1.1 Shape coexistence in the lead region . . . . . . . . . . . . . . . . 59

7.1.2 The $N=126$ shell closure and above . . . . . . . . . . . . . . . . . . 60

7.1.3 Laser spectroscopy of the actinide elements and beyond . . . . . . . . . . . . 62

8 Outlook $\quad 65$

8.1 Recent output . . . . . . . . . . . . . . . . . . . . 65

8.2 Future prospects and challenges . . . . . . . . . . . . . . . 66

8.2.1 Sensitivity and universality . . . . . . . . . . . . . . . 66

8.2 .2 Extraction of nuclear parameters . . . . . . . . . . . . . . 66

8.2 .3 Future experiments and facilities . . . . . . . . . . . . . . 67

9 Conclusions $\quad 68$ 


\section{Introduction}

High-resolution laser spectroscopy has long been established as a powerful tool in the study of nuclear shape, size and multipole structure. The "Hyperfine spectroscopy of radioactive atoms" was first reviewed in 1979 by Jacquinot \& Klapisch [1]. When deployed at radioactive beam facilities, where long chains of isotopes on both sides of the valley of beta stability can be produced, optical techniques still provide a unique opportunity to study changes in the structure of nuclear ground (and isomeric) states.

Otten [2] produced a comprehensive review of the field in 1989. This was updated in 1995 by Billowes \& Campbell [3] and then in 2003 by Kluge \& Nörtershäuser [4]. New experimental techniques, most notably radiofrequency ion beam coolers and bunchers, trapped atoms, and the greatly increased use of specialist ion sources formed the basis of a 2010 review by Cheal \& Flanagan [5].

In this report we review the field with a focus on recent and projected progress with respect to measurements and facility developments, taken as that since the 2010 Cheal \& Flanagan review [5]. Recent reported results, those since 2010, are highlighted in Table 1. Techniques are reviewed in Section 3 and developments at facilities are discussed in Section 4.

The reported results provide model-independent measures of the nuclear spin, multipole moments and radial extent of the charge distribution. The new results are concentrated towards the lighter and heavier mass regions of the nuclear chart. In many of the works new results for nuclear moments are reported and, where of structural pertinence, considered further here. A general review of nuclear moments is not presented. A substantial number of methods, outside of laser spectroscopy, exist for the measurements of these parameters, reviewed by Neugart \& Neyens in 2006 [6]. A complete evaluation of nuclear moment results is maintained by Stone [7], Nuclear Data Section of the IAEA, Vienna. Two further nuclear parameters, the distribution of magnetism and the mass of the nucleus, produce measurable perturbations in the atomic structure. The former gives rise to the so-called "hyperfine anomaly" and is considered in Section 2.2.1. A recent compilation of the measured anomalies has been published by Persson [8]. The latter, the nuclear mass, produces the "mass shift" in atomic spectra, Section 2.3, and requires evaluation for the extraction of nuclear radial parameters. The evaluation requires knowledge of the nuclear mass. Precision measurements of the nuclear mass provide valuable structural information, akin to that provided by charge radii measurements, and are considered further.

A general review of precision atomic physics techniques applied to radioactive nuclear beams (including laser spectroscopy) has recently been made by Blaum, Dilling \& Nörtershäuser [9]. Results presented in that review highlight the close structural connection between mass measurements and charge radii. Moreover the majority of the present and planned Penning-trap mass spectrometers [9] are now, or will be, sited at facilities with on-line laser spectroscopy stations with many sharing common beam preparation traps (Section 4.1). The future possible spectroscopy with such combined stations is considered in Section 8.

The current status of measurements made for contemporary nuclear physics using optical spectroscopic methods is summarised in Fig. 1. The measurements which are currently unpublished are highlighted in green. Table 1 presents an overview of optical measurements as of July 2015 (non-optical measurements are not listed but are discussed where pertinent within this report). References published since the review of Cheal \& Flanagan [5] are indicated separately for clarity. All data published prior to the review of Otten [2], are refered in Table 1 to that review. For earlier data not contained in [2] the original references are provided. In the event of re-evaluation both the re-evaluation and original work are cited. 


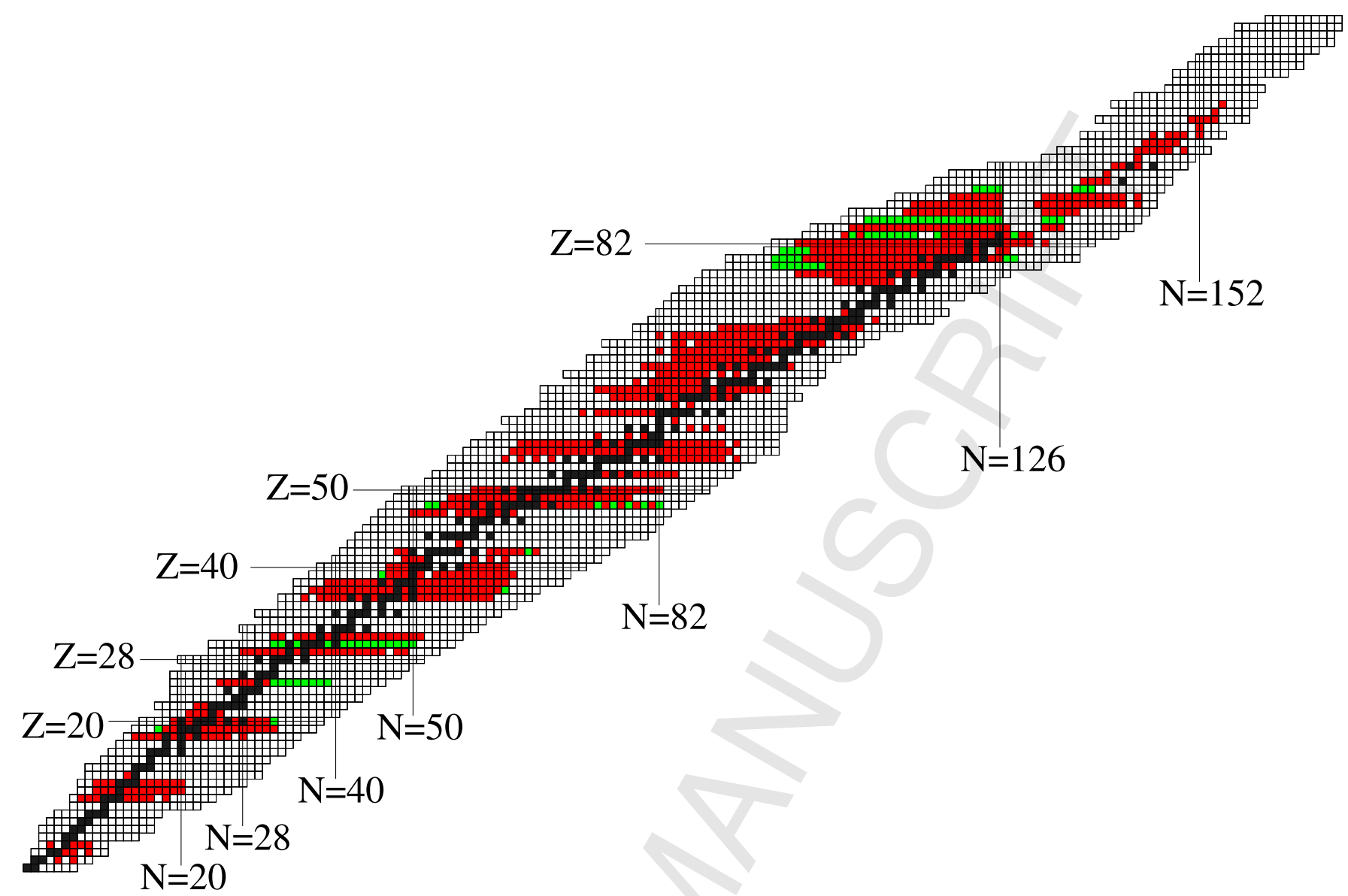

Figure 1: The chart of the nuclides according to optical spectroscopy. Black squares indicate the stable or very long-lived nuclei, red squares indicate optical measurements of radioactive isotopes/isomers. Isotopes coloured green are measurements for which data as of July 2015 are currently unpublished. Colour on-line.

Table 1: Table of optical measurements as of July 2015. References published since the 2010 review of Cheal and Flanagan [5] are highlighted separately in the fourth column.

\begin{tabular}{cclll}
\hline \hline Element & $Z$ & Measured Isotopes & Recent references & Full references \\
\hline $\mathrm{H}$ & 1 & $1,2,3$ & {$[10]$} & {$[10]$} \\
$\mathrm{He}$ & 2 & $3,4,6,8$ & {$[11]$} & {$[11-14]$} \\
$\mathrm{Li}$ & 3 & $6-9,11$ & {$[15,16]$} & {$[2,15-19]$} \\
$\mathrm{Be}$ & 4 & $7,9-12$ & {$[20,21]$} & {$[20-25]$} \\
$\mathrm{Ne}$ & 10 & $17-26,28$ & {$[26]$} & {$[26-28]$} \\
$\mathrm{Na}$ & 11 & $20-31$ & & {$[2,29]$} \\
$\mathrm{Mg}$ & 12 & $21-32$ & {$[30]$} & {$[30,31]$} \\
$\mathrm{Al}$ & 13 & 26,27 & & {$[32,33]$} \\
$\mathrm{Si}$ & 14 & $28-30$ & {$[34]$} & {$[34]$} \\
\hline \hline
\end{tabular}


Table 1. Continued

\begin{tabular}{|c|c|c|c|c|}
\hline Element & $\overline{Z Z}$ & Measured Isotopes & Recent references & Full references \\
\hline $\mathrm{Ar}$ & 18 & $32-44,46$ & & {$[35,36]$} \\
\hline K & 19 & $\begin{array}{l}37-51 \\
38^{m}\end{array}$ & {$[37-42]$} & {$[2,37-44]$} \\
\hline $\mathrm{Ca}$ & 20 & $39-51$ & {$[45]$} & {$[2,45-47]$} \\
\hline $\mathrm{Sc}$ & 21 & $\begin{array}{l}42^{-46} \\
44^{m}, 45^{m}\end{array}$ & [48] & {$[48]$} \\
\hline $\mathrm{Ti}$ & 22 & $44-50$ & & {$[49,50]$} \\
\hline $\mathrm{Cr}$ & 24 & $50,52-54$ & & [51] \\
\hline $\mathrm{Mn}$ & 25 & $\begin{array}{l}50-56 \\
50^{m}, 52^{m}\end{array}$ & {$[52]$} & {$[52,53]$} \\
\hline $\mathrm{Fe}$ & 26 & $54,56-58$ & {$[52]$} & {$[52,54]$} \\
\hline $\mathrm{Ni}$ & 28 & $58,60-62,64$ & & {$[55]$} \\
\hline $\mathrm{Cu}$ & 29 & $\begin{array}{l}57-75,77,78 \\
68^{m}, 70^{m, m 1}\end{array}$ & {$[56,57]$} & {$[56-62]$} \\
\hline $\mathrm{Zn}$ & 30 & $64,66-68,70$ & & {$[63]$} \\
\hline $\mathrm{Ga}$ & 31 & $\begin{array}{l}63,64,66-82 \\
80^{m}\end{array}$ & [64-66] & {$[64-67]$} \\
\hline $\mathrm{Kr}$ & 36 & $\begin{array}{l}72,74-96 \\
79^{m}, 81^{m}, 83^{m}, 85^{m}\end{array}$ & & {$[68,69]$} \\
\hline $\mathrm{Rb}$ & 37 & $\begin{array}{l}74-98 \\
78^{m}, 81^{m}, 82^{m}, 84^{m}, 86^{m}, 90^{m}, 98^{m}\end{array}$ & {$[70-72]$} & {$[2,70-74]$} \\
\hline $\mathrm{Sr}$ & 38 & $\begin{array}{l}77-100 \\
83^{m}, 85^{m}, 87^{m}\end{array}$ & & {$[2,69,75]$} \\
\hline Y & 39 & $\begin{array}{l}86-90,92-99,101,102 \\
87^{m}-90^{m}, 93^{m}, 96^{m}, 97^{m 1, m 2} \\
98^{m}, 100^{m}\end{array}$ & & {$[76,77]$} \\
\hline $\mathrm{Zr}$ & 40 & $\begin{array}{l}87-92,94,96-102 \\
87^{m}, 89^{m}\end{array}$ & & {$[78-80]$} \\
\hline $\mathrm{Nb}$ & 41 & $\begin{array}{l}90-93,99-103 \text { (odds) } \\
90^{m}, 91^{m}\end{array}$ & & [81] \\
\hline Mo & 42 & 90-92, 94-98, 100, 102-106, 108 & & {$[82]$} \\
\hline $\mathrm{Ru}$ & 44 & $96,98-102,104$ & {$[83]$} & {$[83]$} \\
\hline $\mathrm{Pd}$ & 46 & $102,104-106,108,110$ & & {$[84,85]$} \\
\hline $\mathrm{Ag}$ & 47 & $\begin{array}{l}97-101,103-105,107,109 \\
99^{m}, 101^{m}, 104^{m}-106^{m}, 108^{m}, 110^{m}\end{array}$ & {$[86]$} & {$[2,86-88]$} \\
\hline $\mathrm{Cd}$ & 48 & $\begin{array}{l}102-121,123-129 \text { (odds) } \\
111^{m}-129^{m} \text { (odds) }\end{array}$ & [89] & {$[2,89]$} \\
\hline In & 49 & $\begin{array}{l}104-127 \\
108^{m}, 112^{m}, 113^{m}, 115^{m}-126^{m}\end{array}$ & & {$[2]$} \\
\hline $\mathrm{Sn}$ & 50 & $\begin{array}{l}108-132 \\
117^{m}, 121^{m}, 125^{m}-131^{m} \text { (odds), } \\
130^{m}\end{array}$ & & {$[2,90,91]$} \\
\hline $\mathrm{Te}$ & 52 & $\begin{array}{l}120,122-136 \\
123^{m}-133^{m} \text { (odds) }\end{array}$ & & {$[92-94]$} \\
\hline $\mathrm{Xe}$ & 54 & $\begin{array}{l}116-126 \text { (evens), 128-132, 134, } \\
136-144,146\end{array}$ & & {$[2,95]$} \\
\hline
\end{tabular}


Table 1. Continued

\begin{tabular}{|c|c|c|c|c|}
\hline Element & $\overline{Z Z}$ & Measured Isotopes & Recent references & Full references \\
\hline Cs & 55 & $\begin{array}{l}118-146 \\
119^{m}, 121^{m}, 122^{m}, 130^{m} \\
134^{m}-136^{m}, 138^{m}\end{array}$ & & {$[2]$} \\
\hline $\mathrm{Ba}$ & 56 & $\begin{array}{l}120-146,148 \\
127^{m}-137^{m} \text { (odds), } 130^{m}\end{array}$ & & {$[2,96,97]$} \\
\hline La & 57 & $131,135,137-139$ & & {$[98,99]$} \\
\hline $\mathrm{Ce}$ & 58 & 136-148 (evens) & & {$[100,101]$} \\
\hline $\operatorname{Pr}$ & 59 & 141,143 & & [102] \\
\hline $\mathrm{Nd}$ & 60 & $132,134-146,148,150$ & & {$[2,103,104]$} \\
\hline $\mathrm{Pm}$ & 61 & 145,147 & & [105] \\
\hline $\mathrm{Sm}$ & 62 & $\begin{array}{l}138-154 \\
141^{m}\end{array}$ & & {$[2,103,106]$} \\
\hline $\mathrm{Eu}$ & 63 & $\begin{array}{l}137-159 \\
142^{m}, 150^{m}, 152^{m}\end{array}$ & & {$[2,103,107-110]$} \\
\hline $\mathrm{Gd}$ & 64 & $\begin{array}{l}145^{-152,154-158,160} \\
143^{m}, 145^{m}\end{array}$ & & {$[111-114]$} \\
\hline $\mathrm{Tb}$ & 65 & 147-153, 155-159 (odds) & & {$[115]$} \\
\hline Dy & 66 & $\begin{array}{l}146-165 \\
147^{m}\end{array}$ & & {$[2,116]$} \\
\hline Ho & 67 & $\begin{array}{l}151-163,165 \\
151^{m}-154^{m}, 156^{m}-162^{m} \text { (eve }\end{array}$ & & {$[2]$} \\
\hline Er & 68 & $150,152-165,167$ & & {$[2]$} \\
\hline $\mathrm{Tm}$ & 69 & $\begin{array}{l}153,154,156-172 \\
154^{m}\end{array}$ & & {$[2,117]$} \\
\hline $\mathrm{Yb}$ & 70 & $\begin{array}{l}152,154-177 \\
176^{m}, 177^{m}\end{array}$ & & {$[2,117-121]$} \\
\hline $\mathrm{Lu}$ & 71 & $\begin{array}{l}161-179 \\
166^{m 1, m 2}, 167^{m}-169^{m}, 171^{m} \\
172^{m}, 174^{m}, 176^{m}-178^{m}\end{array}$ & & {$[122]$} \\
\hline Hf & 72 & $\begin{array}{l}170-180,182 \\
171^{m}, 178^{m 1, m 2}\end{array}$ & & {$[120,123-127]$} \\
\hline $\mathrm{Ta}$ & 73 & $\begin{array}{l}179,181 \\
180^{m}\end{array}$ & & {$[128,129]$} \\
\hline $\mathrm{W}$ & 74 & $180,182-184,186$ & [130] & {$[130]$} \\
\hline $\operatorname{Re}$ & 75 & 185,187 & & {$[131,132]$} \\
\hline Os & 76 & $184,186-190,192$ & & [133-135] \\
\hline Ir & 77 & $\begin{array}{l}182-189,191,193 \\
186^{m}\end{array}$ & & {$[136]$} \\
\hline $\mathrm{Pt}$ & 78 & $\begin{array}{l}178-196,198 \\
183^{m}, 185^{m}\end{array}$ & & {$[2,137-139]$} \\
\hline $\mathrm{Au}$ & 79 & $\begin{array}{l}183-199 \\
184^{m}, 189^{m}\end{array}$ & & {$[2,139-141]$} \\
\hline $\mathrm{Hg}$ & 80 & $\begin{array}{l}181-206 \\
185^{m}-199^{m} \text { (odds) }\end{array}$ & & {$[2]$} \\
\hline $\mathrm{Tl}$ & 81 & $\begin{array}{l}183,185,190-205,207,208 \\
184^{m}-198^{m}, 186^{m 2}\end{array}$ & {$[142,143]$} & {$[2,142,143]$} \\
\hline
\end{tabular}


Table 1. Continued

\begin{tabular}{cclcl}
\hline \hline Element & $Z$ & Measured Isotopes & Recent references & Full references \\
\hline $\mathrm{Pb}$ & 82 & $182-212,214$ & {$[2,144-147]$} \\
& & $183^{m}-189^{m}$ (odds), & \\
& & $193^{m}-197^{m}$ (odds), 202 & \\
$\mathrm{Bi}$ & 83 & $189,191,202-210,212,213$ & & {$[88,148-150]$} \\
& & $191^{m}, 210^{m}$ & & \\
$\mathrm{Po}$ & 84 & $191-211,216,218$ & & {$[151-155]$} \\
& & $191^{m}-203^{m}($ odds $)$ & {$[151-154]$} & \\
$\mathrm{Rn}$ & 86 & $200-202,204-212,218-223,225$ & & {$[2,156]$} \\
& & $203^{m}$ & & {$[2,157-165]$} \\
$\mathrm{Fr}$ & 87 & $202-213,219-229,231$ & \\
& & $202^{m}, 204^{m 1, m 2}, 206^{m 1, m 2}, 218^{m}$ & {$[157-162]$} & {$[2,166]$} \\
$\mathrm{Ra}$ & 88 & $208-214,220-230,232$ & {$[167-170]$} \\
$\mathrm{Th}$ & 90 & $227-230,232$ & {$[171]$} \\
$\mathrm{Pa}$ & 91 & 231 & {$[167-169]$} & {$[172-174]$} \\
$\mathrm{U}$ & 92 & $233-236,238$ & {$[175]$} \\
$\mathrm{Np}$ & 93 & 237 & & {$[176]$} \\
$\mathrm{Pu}$ & 94 & $238-242$ & {$[177-184]$} \\
$\mathrm{Am}$ & 95 & $240,241,243$ & \\
$\mathrm{Cm}$ & 96 & $242,244-246,248$ & {$[185,186]$} \\
$\mathrm{Bk}$ & 97 & 249 & {$[186,187]$} \\
$\mathrm{Cf}$ & 98 & $249-252$ & {$[188]$} \\
$\mathrm{Es}$ & 99 & 253,255 & {$[189,190]$} \\
$\mathrm{Fm}$ & 100 & 255 & & \\
\hline \hline
\end{tabular}




\section{Nuclear perturbations of the atomic structure}

In an atomic nucleus, with atomic spin $\hat{J}$, nuclear spin $\hat{I}$ and total angular momentum $\hat{F}=\hat{I}+\hat{J}$, spectroscopic measurements of transition frequencies may be attempted between states involving electronic transitions (most typically, electric dipole) or within the same atomic state (magnetic dipole) or, in the presence of an external field, between substates, $m_{J}, m_{F}$, of these levels. The following considerations are discussed in the context of the former (electronic) transitions. Spectroscopy within the hyperfine multiplet, defined below, is discussed in Section 2.2.1.

As highlighted in the introduction, the nuclear spin, multipole moments, radial extent of the charge distribution, distribution of magnetism and the mass produce (measurable) perturbations of the atomic structure. The interactions that give rise to these perturbations and the methods used to extract nuclear observables from their measurement are described below.

\subsection{Nuclear ground state properties from atomic spectra}

The Hamiltonian describing the interaction between an atomic nucleus and its surrounding electromagnetic fields may be written as [192]

$$
H=\sum_{k} \underline{\hat{T}}^{k}(N) \otimes \underline{\hat{T}}^{k}(E)
$$

where the irreducible tensors $\underline{\hat{T}}^{k}(N)$ and $\underline{\hat{T}}^{k}(E)$ are of rank $k$ and describe the electromagnetic properties of the nucleus and surroundings, respectively. Parity conservation considerations of the strong and electromagnetic interactions dictate that the general operator $\hat{T}_{q=0}^{k}$ consists of negative parity magnetic and positive parity electric multipole operators. It therefore follows that the nucleus can be described by a series of even- $k$ electric (monopole, quadrupole, ...) and odd- $k$ magnetic (dipole, octupole, ...) multipole moments up to order $k$ where $k \leq 2 I$. The use of laser spectroscopic techniques, whilst theoretically sensitive to the entire multipole expansion of the nuclear extent, is generally limited to moments of order $k \leq 2$. The rapidly decreasing magnitude of terms in the expansion, in comparison to the uncertainties associated with measurements, lead to this rapid exhaustion.

\subsection{Hyperfine structure}

The atomic hyperfine structure arises from the Hamiltonian given in equation 1 where the electromagnetic tensor is derived from the field and field gradients of the atomic electron cloud and the nuclear tensor from the multipole expansion of the nuclear moments. Evaluation of the interaction energy is most often treated as a perturbation to the electronic fine structure, with both magnetic and quadrupole terms being of order $\mu \mathrm{eV}$ (in comparison to the $\mathrm{eV}$ scale of the fine structure splitting). In systems exhibiting small fine structure splittings, encountered at half-filled shells or in Rydberg states, secondorder perturbations can be considered, for example as in reference [129], or, when the splittings are comparable in magnitude, simultaneous diagonalization performed, such as that in reference [119].

Including only those terms arising from the nuclear magnetic dipole and electric quadrupole moments, the energy shift for each atomic hyperfine level with respect to the unperturbed fine structure level is given by,

$$
\begin{aligned}
\Delta E_{H F S} & =\Delta E_{\text {dipole }}+\Delta E_{\text {quadrupole }} \\
& =\frac{A}{2} C+\frac{B}{4} \frac{\frac{3}{2} C(C+1)-2 I J(I+1)(J+1)}{I J(2 I-1)(2 J-1)}
\end{aligned}
$$


where $C=F(F+1)-J(J+1)-I(I+1)$, and $A$ and $B$ are the hyperfine coefficients which are dependent on both nuclear and atomic structural parameters. Following this definition the magnetic hyperfine coefficient is given by $A=\frac{\mu B_{e}(0)}{I J}$, where $\mu$ is the nuclear magnetic dipole moment and $B_{e}(0)$ the magnetic field generated at the nuclear site due to the atomic electrons. The electric coefficient is given by $B=e Q_{s}\left\langle\frac{\partial^{2} V}{\partial z^{2}}\right\rangle$ with $Q_{s}$ being the spectroscopic quadrupole moment and the time-averaged partial differential being the average electric field gradient generated by the electrons at the nucleus.

Although a measurement of the absolute values of $\mu$ and $Q_{s}$ can be obtained following a precise calculation of the relevant electromagnetic fields (with modern calculations possible for even high- $Z$ nuclei [193]) it is more common practice to extract the ratio of the nuclear moments of a pair of different isotopes of the same element using the approximate relationships,

$$
\frac{A}{A^{\prime}} \simeq \frac{\mu}{\mu^{\prime}} \frac{I^{\prime}}{I} \quad \frac{B}{B^{\prime}} \simeq \frac{Q_{s}}{Q_{s}^{\prime}}
$$

Knowledge of the moments of a reference isotope, measured for example using Nuclear Magnetic Resonance (NMR) [6, 194], thus permits an approximate scaled extraction. The uncertainty introduced on scaling depends on the differential change in the distribution of nuclear charge and magnetism. For quadrupole moments, where typical evaluations have at best $\sim 1 \%$ precision [195], no experimental reports of differential changes are available (and reference [195] suggests a unified approach for future calibrations). For magnetic moments, the differential changes are referred to as "anomalies" and are considered in the next section.

The dependence of the hyperfine splitting on nuclear spin $I$ ensures that, given enough splittings exist, a unique assignment of the nuclear spin can be made. Experimentally, inspecting data from zero-field hyperfine splittings, it can be found that within uncertainties only lower limits can be placed on the value of $I$, a problem especially encountered in systems with $I>J$.

\subsubsection{Hyperfine anomalies}

The relationship for the $A$-coefficients in equation 4 is exact for an infinitely small nucleus. In real systems, especially the heavier nuclei, the finite nuclear extent and relativistic effects have bearing on the electronic wavefunctions. Two additional mechanisms give rise to corrections that must be considered. These are the Breit-Rosenthal effect (BR) [196], which arises from the finite spatial distribution of the nuclear charge, and the Bohr-Weisskopf effect (BW) [197], from the distribution of nuclear magnetism within the nucleus. Due to the small size of these contributions they are commonly accounted for by the addition of two factors,

$$
A=A_{\text {point }}\left(1+\epsilon_{B W}\right)\left(1+\epsilon_{B R}\right) .
$$

Here $A_{\text {point }}$ is the hyperfine $A$ coefficient for a theoretical, point-like nucleus. A commonly used parameter is the differential anomaly ${ }^{A} \Delta^{A^{\prime}}$ resulting from the ratio of $A$ coefficients between two isotopes,

$$
\frac{A}{A^{\prime}}=\frac{A_{\text {point }}\left(1+\epsilon_{B W}\right)\left(1+\epsilon_{B R}\right)}{A_{\text {point }}^{\prime}\left(1+\epsilon_{B W}^{\prime}\right)\left(1+\epsilon_{B R}^{\prime}\right)} \approx \frac{\mu I^{\prime}}{\mu^{\prime} I}\left(1+{ }^{A} \Delta^{A^{\prime}}\right)
$$

A recent compilation of differential anomalies can be found in reference [8]. Direct observation of magnetic hyperfine transitions, within a hyperfine multiplet, can be made using radio-frequency resonance spectroscopy with extremely high precision. Such spectroscopy, combined with knowledge of the nuclear $g$-factor, readily provides high accuracy measurements of ${ }^{A} \Delta^{A^{\prime}}$. Typically the value is found to be small relative to experimental uncertainties, $<1 \%$, but is observed to rise to close to $10 \%$ in some (limited) cases [8]. 


\subsection{Isotope shift}

The frequency of the same atomic transition is observed to shift between different isotopes of the same element. The effect, known as the isotope shift, arises due to changes in the nuclear mass and size. For nuclei with atomic masses $A$ and $A^{\prime}$ it is generally defined as,

$$
\delta \nu^{A, A^{\prime}}=\nu^{A^{\prime}}-\nu^{A},
$$

where $\nu^{A}$ is the frequency of the atomic transition for isotope $A$. In general this can be approximated as being composed of two independent components, the Mass shift arising from the change in total mass of the system and the Field shift arising from the change in the spatial distribution of the nuclear charge [198]. Equation 7 can therefore be rewritten as,

$$
\delta \nu^{A, A^{\prime}}=\delta \nu_{\text {mass shift }}^{A, A^{\prime}}+\delta \nu_{\text {field shift }}^{A, A^{\prime}} .
$$

The mass shift component is separable into two components, the Normal Mass Shift equivalent to the Bohr reduced mass correction and the Specific Mass Shift arising from the electron-electron correlations within a multi-electron system. The mass shift can be written as

$$
\delta \nu_{\text {mass shift }}^{A, A^{\prime}}=\frac{m^{A^{\prime}}-m^{A}}{m^{A} m^{A^{\prime}}}(N+S),
$$

where $N$ and $S$ are the constants for the normal and specific mass shifts, respectively. The normal mass shift constant, $N$, is exactly calculable whereas the specific mass shift constant, $S$, must either be theoretically evaluated or calibrated [198]. A recent summary and case studies for evaluation of $S$, covering many of the elements discussed in this report, is presented in reference [199]. Calculations of the parameter for the lightest nuclei are detailed in the next section. Experimental evaluation of $S$ is possible using evaluations of nuclear size changes independent to optical data. From the data it may be seen that the absolute magnitude of $S$ is typically less than that of $N$ [200] but can rise significantly in elements with partially filled atomic d-shells.

Evaluation of $S$ and knowledge of the nuclear masses permits extraction of the field-shift component for any $A, A^{\prime}$ pair. Experimentally the relative size of the field and mass shift changes by some 7 orders of magnitude over the nuclei considered in this report. The changing ratio of these effects is highlighted in each of the individual result sections. In the light mass nuclei the mass shift dominates, often by many orders of magnitude, in the heaviest nuclei the relative sizes are reversed. Around $Z \sim 30$ comparable field and mass shift are found (for transitions involving a change in the number of valence $s$-electrons, see below).

The field shift arises from a perturbation of binding energy that results from the spatial overlap of the electron wavefunction, $\psi_{e}(r)$, and the nuclear volume. The difference in this energy shift between two isotopes can be expressed as

$$
\delta E=e \int_{0}^{\infty} \psi_{e}^{*}(r) \delta V(r) \psi_{e}(r) d^{3} r
$$

where $\delta V(r)$ is the change in the Coulomb potential between the two isotopes. Under the assumption that the electronic wavefunction is constant across the nuclear volume this results in a change in the frequency of an observed transition given by

$$
\delta \nu_{\text {field shift }}^{A, A^{\prime}}=\frac{Z e^{2}}{6 h \epsilon_{0}} \Delta\left|\psi_{e}(0)\right|^{2} \delta\left\langle r^{2}\right\rangle^{A, A^{\prime}}
$$

Here the $\Delta\left|\psi_{e}(0)\right|^{2}$ term corresponds to the change in electron density at the nucleus between the two atomic levels comprising the transition and $\delta\left\langle r^{2}\right\rangle$ is the change in the mean-square charge radii between 
the two isotopes. The dependency on $\Delta\left|\psi_{e}(0)\right|^{2}$ results in transitions in which, if the number of $s$ electrons in the valence orbital changes, produces large field shifts (these electrons, and relativistic $p_{1 / 2}$ electrons, having finite $\left.\left|\psi_{e}(0)\right|^{2}\right)$.

The approximation of a constant electron wavefunction across the nuclear volume in equation 11 becomes significantly less valid with increasing atomic number. The field shift is therefore more commonly and more generally expressed as

$$
\delta \nu_{\text {field shift }}^{A, A^{\prime}}=F(Z) \lambda^{A, A^{\prime}}
$$

where $F(Z)$ contains the optical transition dependence and $\lambda^{A, A^{\prime}}$ is the Seltzer moment [201] which is described by

$$
\lambda^{A, A^{\prime}}=\delta\left\langle r^{2}\right\rangle^{A, A^{\prime}}+b_{1} \delta\left\langle r^{4}\right\rangle^{A, A^{\prime}}+b_{2} \delta\left\langle r^{6}\right\rangle^{A, A^{\prime}}+\ldots=k \delta\left\langle r^{2}\right\rangle^{A, A^{\prime}} .
$$

The parameterization of the expectation values of higher even order moments in terms proportional to $\delta\left\langle r^{2}\right\rangle^{A, A^{\prime}}$ acts to recover a simple proportionality to changes in the mean-square radius.

Calculation or calibration of the atomic factor, $F(Z)$, is required to extract the nuclear parameter. For many years calibration using other sources of $\delta\left\langle r^{2}\right\rangle^{A, A^{\prime}}$, for example the results of electron scattering experiments, muonic and X-ray isotope shifts, have been invoked [2, 200]. Where such data does not exist, often for elements with one or less stable isotopes, semi-empirical estimation or theoretical calculation of the factor have been the only possible approaches. In the former both the gross structure of alkali-like atoms and the hyperfine splittings of s-electron configurations can be used to provide estimates of $\left|\psi_{e}(0)\right|^{2}[2,198]$. Semi-empirical estimates [2] or calculation are then invoked to estimate the total electron density change in the transition, $\Delta\left|\psi_{e}(0)\right|^{2}$. More recently [199] direct theoretical calculation of the factor, using large-scale Dirac-Fock frameworks, have been favoured. The estimates such ab-initio calculations produce are typically cited as accurate to $15 \%$ [199]. The global systematics of extracted $\delta\left\langle r^{2}\right\rangle^{A, A^{\prime}}$, discussed in the following sections, would appear to suggest that this represents an overestimate of the uncertainty. Beyond $Z \sim 36$, few, if any, of extracted (and extensive) chains of isotopic $\delta\left\langle r^{2}\right\rangle^{A, A^{\prime}}$ show substantial differences to the trends displayed in neighbouring isotope chains, see Section 8 . While discrepancies at the $\sim 5 \%$ level may still persist the theoretical estimates appear to sit favourably with those calibrated by other means, compatible with smooth global trends. Moreover, when subjected to rigorous comparison for different transitions within the same element the calculations again perform well [199] and enhanced confidence would appear reasonable.

The extracted value of $\delta\left\langle r^{2}\right\rangle$ is, importantly, independent of nuclear model. This property of the optically determined observable separates it from the majority of (non-optical) measures of the nuclear charge distribution.

\subsection{Mean-squared nuclear charge radii}

If it is assumed that there is no spatial distinction between constituent nucleons a liquid drop model approach with a homogeneous distribution will result in a mean-square charge radius of

$$
\left\langle r^{2}\right\rangle=\frac{3}{5} r_{0}^{2} A^{\frac{2}{3}}
$$

where $r_{0} \approx 1.2 \mathrm{fm}$ and $A$ is the atomic mass. For a general distribution with charge density function $\rho(\vec{r})$ the mean-square radius is defined by

$$
\left\langle r^{2}\right\rangle=\frac{\int_{0}^{\infty} \rho(\vec{r}) r^{2} d^{3} r}{\int_{0}^{\infty} \rho(\vec{r}) d^{3} r}
$$


(the denominator representing the total nuclear charge, Ze). Expansion of a deformed distribution in terms of the spherical harmonics modifies the expectation such that

$$
\left\langle r^{2}\right\rangle=\left\langle r^{2}\right\rangle_{0}\left(1+\frac{5}{4 \pi} \sum_{i=2}^{\infty}\left\langle\beta_{i}^{2}\right\rangle\right),
$$

which is commonly approximated to be

$$
\left\langle r^{2}\right\rangle \approx\left\langle r^{2}\right\rangle_{0}\left(1+\frac{5}{4 \pi}\left\langle\beta_{2}^{2}\right\rangle\right)
$$

where $\left\langle r^{2}\right\rangle_{0}$ is the mean-square charge radius of a spherical nucleus of identical volume and $\beta_{i}$ are the deformation parameters of order $i$. When modelled by the convolution of a uniform "sharp cut off" distribution with a Gaussian, the diffuseness of the nuclear surface is found to produce a constant additive contribution to equation 16. The term in the model is independent of $A, Z, N$ and $\beta_{i}$ and, despite its surprising appearance, is supported by experimental measurement. A constant contribution arising from the diffuseness, irrespective of the nucleus, close to $3 \mathrm{fm}^{2}$ is compatible with electron scattering results [202]. The change in $\left\langle r^{2}\right\rangle$ between two isotopes with masses $A$ and $A^{\prime}$ is thus given to second order by

$$
\delta\left\langle r^{2}\right\rangle^{A, A^{\prime}}=\delta\left\langle r^{2}\right\rangle_{0}^{A, A^{\prime}}+\left\langle r^{2}\right\rangle_{0} \cdot \frac{5}{4 \pi} \delta\left\langle\beta_{2}^{2}\right\rangle^{A, A^{\prime}}
$$

The appearance of the $\delta\left\langle\beta_{2}^{2}\right\rangle^{A, A^{\prime}}$ parameter in the above has provided one of the greatest motivations for the optical spectroscopy of the nucleus and one of the fields' most important, and fruitful, connections to the general field of nuclear spectroscopy. At $A \sim 100$ the $\delta\left\langle r^{2}\right\rangle$ arising from the size increase of the nucleus on the addition of a neutron may be estimated to be $\sim 0.07 \mathrm{fm}^{2}$. If the neutron affects the structure such that the shape changes from $\left\langle\beta_{2}^{2}\right\rangle=0$ to $\left\langle\beta_{2}^{2}\right\rangle=0.1$ a corresponding change in $\delta\left\langle r^{2}\right\rangle$ of $\sim 0.74 \mathrm{fm}^{2}$, an order of magnitude larger, can be expected. The extreme sensitivity of $\delta\left\langle r^{2}\right\rangle$ to changes in the nuclear shape, the model-independence of its extraction and the isotopes in which it can be measured have resulted in a substantial experimental effort being directed to optical nuclear spectroscopy.

\subsection{Relation to other nuclear observables}

The experimental spectroscopic quadrupole moment $\left(Q_{s}\right)$ may be projected to the intrinsic quadrupole moment $\left(Q_{0}\right)$ under the assumption of a well-defined deformation axis (the strong-coupling limit),

$$
Q_{s}=\frac{3 \Omega^{2}-I(I+1)}{(I+1)(2 I+3)} Q_{0}
$$

where $\Omega$ is the projection of $I$ on the deformation axis. This intrinsic moment is related to the quadrupole deformation parameter $\left(\beta_{2}\right)$ via

$$
Q_{0}=\frac{3}{\sqrt{5 \pi}} Z e R^{2}\left\langle\beta_{2}\right\rangle\left(1+0.36\left\langle\beta_{2}\right\rangle\right)
$$

where $R$ is the radius of a homogeneous sphere of mass $A$. As such, albeit with model dependence on the coupling and choice of spherical radius, a measure of the static quadrupole deformation, $\left\langle\beta_{2}\right\rangle$, may be derived. Results for changes in charge radii afford measures of changes in mean-square quadrupole deformation, $\left\langle\beta_{2}^{2}\right\rangle$, and contain contributions from both changes in static and dynamic quadrupole deformation. 
A number of nuclear, often $\gamma$-ray based, spectroscopy infer results concerning the deformation of the nucleus. The observations of rotational band structures, nuclear isomerism (notably fission isomers) and changes in reaction cross-sections are interpreted in terms of nuclear deformation. Two nuclear observables, transition probabilities and the nuclear mass, are especially measured in explorations of nuclear shape. The latter is discussed explicitly later. Transition probabilities, particularly those from the ground state to the first excited $2^{+}$state $(\mathrm{B}(\mathrm{E} 2)$, in even-even nuclei), show strong correlation with the nuclear deformation. The $\mathrm{B}(\mathrm{E} 2)$ transition probability [203] is directly proportional to $\left\langle\beta_{2}^{2}\right\rangle$ and frequently discussed in the interpretation of mean-square charge radii measurements. Recent comparisons of behaviour of these different nuclear observables have presented for the medium to heavy mass regions by Cakirli et al. [204] and for lighter nuclei $(Z \leq 16)$ by Angeli and Marinova [205], with the latter evaluating isotonic (as well as isotopic) trends using charge radii data. 


\section{Atomic Spectroscopy}

\subsection{Excitation and detection of atomic transitions}

The atomic spectroscopy reviewed in this report has been performed using laser light at frequencies spanning the infra-red to ultraviolet regions of the electromagnetic spectrum. The spectroscopy is in a number of studies combined with radio-frequency excitation (within a hyperfine multiplet) and with non-optical (de-)excitation mechanisms not involving photons (for example, collisional or thermal excitation, field ionisation or charge exchange). The sources of the laser light used are both continuous and pulsed, single and multi-mode and are frequently manipulated using non-linear optical techniques such as frequency doubling, tripling and mixing.

The optical (laser) spectroscopy primarily exploits the use of allowed electric dipole transitions between two hyperfine states in different fine structure levels. In the absence of stimulated effects or feeding from higher lying electronic levels the absolute intensities of the different transitions is dependent upon the angular momenta of the constituent hyperfine levels in addition to the total line strength of the unperturbed fine structure transition, $S_{J J^{\prime}}$, such that

$$
S_{F F^{\prime}}=(2 F+1)\left(2 F^{\prime}+1\right)\left\{\begin{array}{ccc}
F & F^{\prime} & 1 \\
J^{\prime} & J & I
\end{array}\right\}^{2} S_{J J^{\prime}}
$$

where $\{\ldots\}$ is a Wigner $6-j$ symbol. As such, from the $I$ dependence, the relative intensities observed in a hyperfine structure pattern can aid in the assignment of the nuclear spin (described in Section 2.2). Typically, in the reviewed work, dipole transitions from the electronic ground or long-living metastable states are studied and only the finite lifetime of the upper state determines the absorption profile of isolated atoms at rest. The absorption rate, $\gamma_{p}$, in response to excitation from a monochromatic light field at frequency $\delta$ relative to the line centre is then given by

$$
\gamma_{p}=\frac{s_{0} \frac{\Gamma}{2}}{1+s_{0}+\left(\frac{2 \delta}{\Gamma}\right)^{2}}
$$

where $\Gamma$ is the natural linewidth of the excited electronic state and $s_{0}$ is the on-resonance saturation parameter. The saturation parameter $s_{0}=I / I_{s}$ where $I$ is the intensity of the exciting radiation and $I_{s}$ is the saturation intensity defined as

$$
I_{s} \equiv \frac{\pi h c}{3 \lambda^{3} \tau}
$$

with $\lambda$ the wavelength of the transition and $\tau$ the lifetime of the electronic state. Magnetic dipole transitions, excited in radiofrequency transitions within the hyperfine sub-levels, have the same wavelength (and thus energy) dependence and for these states, where spontaneous emission occurs with negligible probability, excited state lifetimes are dominated by spin-spin and spin-lattice de-excitation processes.

Higher multipole transitions are strongly suppressed in comparison to electric dipole transitions (of the same energy) in the atom. Multi-photon (de-)excitation processes are instead encountered preferentially and of these transmutation involving two photons feature in a number of the described studies. An experimentally attractive feature of this transmutation as an excitation mechanism arises from the cancellation of (non-relativistic) first order Doppler shifts if two, counter-propagating, photons of half the transition energy are used to excite the atom. The general suppression of Doppler (and other) broadening mechanisms is a common feature in the majority of the discussed spectroscopy. Suppression improves the spectroscopic resolution but, often more critically, facilitates efficient sample use by ensuring all members of an atomic ensemble are simultaneously on resonance during a measurement.

The detection of atomic resonance may be achieved in a variety of manners around which a range of spectroscopies have been developed. Each is performed against different backgrounds and random 
interferences to the resonance signal. The spectroscopy is often tailored to a particular chemical species or to the nature of the production facility (Section 4). The studies reported in Table 1 have involved detection via observation of resonance fluorescence, optical pumping, laser-induced nuclear orientation and resonance ionisation. We review each in this section focusing on the elements (and isotopes) studies to which the techniques have been applied.

\section{$3.2 \quad$ Pioneering experiments}

The insight optical spectroscopy could provide in the explorations of the nuclear structure was spectacularly demonstrated by the optical-pumping studies of Hg at ISOLDE by Bonn et al. [206] in 1972. These experiments were performed before the availability of the narrow-linewidth tunable laser, later realised by Hänsch [207]. The pioneering developments, and the range of techniques they produced, expanded rapidly from 1972. Optical-pumping experiments at the Karlsruhe cyclotron [208] and, using laser light, at the Orsay synchrocyclotron [209] pioneered optical techniques at accelerators and the first on-line collinear beam experiments [210] were reported at the TRIGA reactor, Mainz in 1978.

\subsection{Sample preparation}

The presently operational (and planned) primary production platforms of the radioactive nuclei reviewed here are discussed in Section 4. A wide range of primary nuclear reactions are in use at the various on-line separators described in that section and are summarized there in Table 3 (with, the italicised, production terminology followed here). Fusion-evaporation reactions using light-ion and heavy-ion projectiles have enabled production of neutron-deficient nuclei close to the proton dripline for elements as heavy as ${ }^{185} \mathrm{Bi}$ [211]. Charged-particle induced fission, fast fission and fragmentation $(F F)$ have provided a wealth of neutron-rich and neutron-deficient species. On-line access to isotope chains spanning more than 20 isotopes has been possible for medium to heavy mass nuclei. Spontaneous-, neutron- and photo-induced fission provide a cooler fissioning system, suffering less neutron evaporation, and produce nuclei at extreme neutron-excess with the highest cross-sections. In-flight fragmentation provides high-energy exotic nuclear production at both extremes of neutron-excess and neutron-deficiency.

A range of secondary ion sources, described in connection with individual facilities (sections 4.1.1 4.1.14), are presently used to produce low energy, $10-100 \mathrm{keV}$, ion beams or ensembles from (usually high energy) reaction products. Thermal and arc-discharge (often FEBIAD, forced electron beam induced-arc discharge) ion sources $[212,213]$ are capable of producing low-energy spread, $<1 \mathrm{eV}$, beams for all but the most refractory elemental species. Laser ion sources, LIS [214], combine this broad production with the selectivity of laser ionisation to critically assist the selectivity of the secondary source. Ion guide sources [215] facilitate a chemically insensitive production of short-lived species, albeit often at large initial energy spread. In the technique nuclear recoils are stopped in flowing (He) gas from which ions are electrostatically skimmed and extracted to high vacuum acceleration regions. Gas cell stoppers [216-218] operate in a similar manner to the ion guide but on an increased scale and decreased gas flow. Thermalised ions in such cells are guided through the gas, using combined static and RF fields, prior to jet extraction. Laser ion guide sources, for example LISOL [219, 220], couple again laser selectivity to the (ion guide) source. Laser ion source traps, LIST [221, 222], combine efficient atom sources with radio-frequency quadrupole ion traps to provide ion sources of the highest selectivity. All of the laser-assisted sources provide spectroscopic opportunities directly within the source and their descriptions are included in the spectroscopies described in this section. 


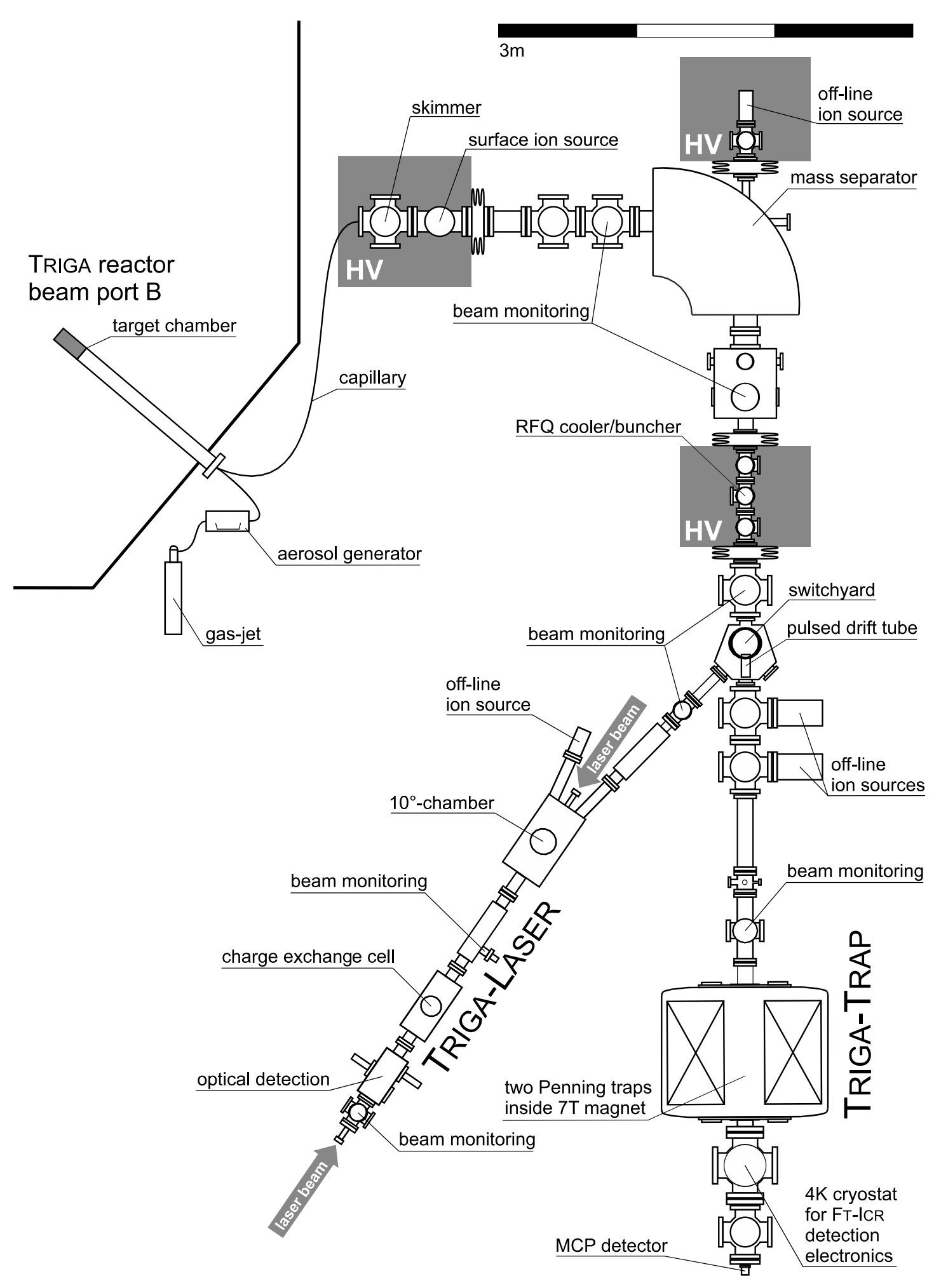

Figure 2: Layout of the TRIGA-SPEC facility, Mainz [223]. (Figure courtesy of Wilfried Nörtershäuser.) 


\subsection{Collinear Laser Spectroscopy}

Spectroscopy based on a combination of lasers and fast ion beams can be traced to as early as 1975 and the work of Andrä [224]. Work in a collinear geometry originates however from the experimental and theoretical efforts of Wing et al. [225] and Kaufman [226], with the latter rapidly resulting in experiments of Anton et al. [227]. The first on-line application, Schinzler et al. [210], shortly followed and immediately demonstrated the efficacy of the technique when applied directly at on-line isotope separators. In a collinear geometry, light, whether co- or counter-propagating with the ion beam, interacts with accelerated ionic ensembles that experience a velocity compression along the axis of their motion. The compression arises from an acceleration to well-defined energy $E$ preserving an original energy spread $\delta E$ but reducing the corresponding velocity spread to,

$$
\delta v=\frac{1}{\sqrt{2 m E}} \delta E .
$$

Typical secondary ion sources, whether thermal (surface ionisation), FEBIAD or gas cell constructions, provide ionic ensembles with energy spreads of at least $1 \mathrm{eV}$ (and often far higher in the case of gas stoppers or ion guides). On acceleration to energies of order $30 \mathrm{keV}$, singly-charged ionic species in the medium mass region (taken for all estimates to be $A=100$ ) produce a $\sim 4 \mathrm{~ms}^{-1}$ velocity spread for a $1 \mathrm{eV}$ initial energy spread. Compared to an unaccelerated ensemble, where a velocity spread of some $1500 \mathrm{~ms}^{-1}$ would be observed, a 3 order of magnitude compression is achieved. For a $300 \mathrm{~nm}\left(10^{15}\right.$ $\mathrm{Hz}$ ) transition the compressed velocity spread corresponds to a residual Doppler broadened width of $\sim$ $30 \mathrm{MHz}$ readily comparable to the natural broadening observed for a state of lifetime $5 \mathrm{~ns}$. As such, acceleration to at least $30 \mathrm{keV}$ reduces ion source broadenings to a level beneath those that arise from typical atomic state lifetimes.

As will be seen in Section 3.8, collinear laser spectroscopy has been used to study the vast majority of the species cited in Table 1. Many variants of the fast beam technique have been developed (nine sub-categories are considered in Section 3.8) and a wide variety of resonance detection strategies are now used to facilitate the spectroscopy. An experimental realisation of the technique is shown in Figure 2. The figure shows the layout of the TRIGA-SPEC facility, Mainz [223], where one of two spectroscopy lines is dedicated to laser studies (and both are fed by a common RFQ cooler-buncher, Section 3.4.3). Acceleration is achieved with ion sources and bunchers (on-line ion traps) at precisely stabilized positive high potential from which ions emerge to grounded experimental stations. The source is fed with radioactive fission fragments and the extracted fast beam is mass-analyzed and a desired isotopic beam overlapped with laser light.

Collinear spectroscopy is attempted at speeds in excess of $0.1 \% c$. At such speeds interaction lengths of $10 \mathrm{~mm}$ correspond to timescales an order of magnitude greater than typical lifetimes and imaged, or condensed, light collected from an overlap region of this size can permit resonance fluorescence detection at efficiencies of $>1 \%$. The dimension of the interaction region matches readily that of the photocathode size on commercially available, single-photon counting, multipliers.

A more detailed view of a spectroscopy line, following that of the station described in Section 4.1.7, is shown in Figure 3. In the schematic a general purpose radio-frequency quadrupole trap delivers continuous or bunched ionic ensembles to a number of experimental stations including a collinear laser spectroscopy line. Ionic ensembles are overlapped with counter-propagating laser light in a velocity (Doppler) tunable beam line section. The overlap region is imaged on to the photocathode of a segmented photomultiplier tube. Resonantly and randomly scattered photons are detected by the position sensitive photomultiplier. Downstream ion and decay radiation detectors facilitate coincidence and decay-tagged spectroscopy.

The sensitivity of the spectroscopy is limited by backgrounds from uncorrelated laser light scatter, photomultiplier dark or radiation-induced counts and light from fast ion collisions with residual-gas 


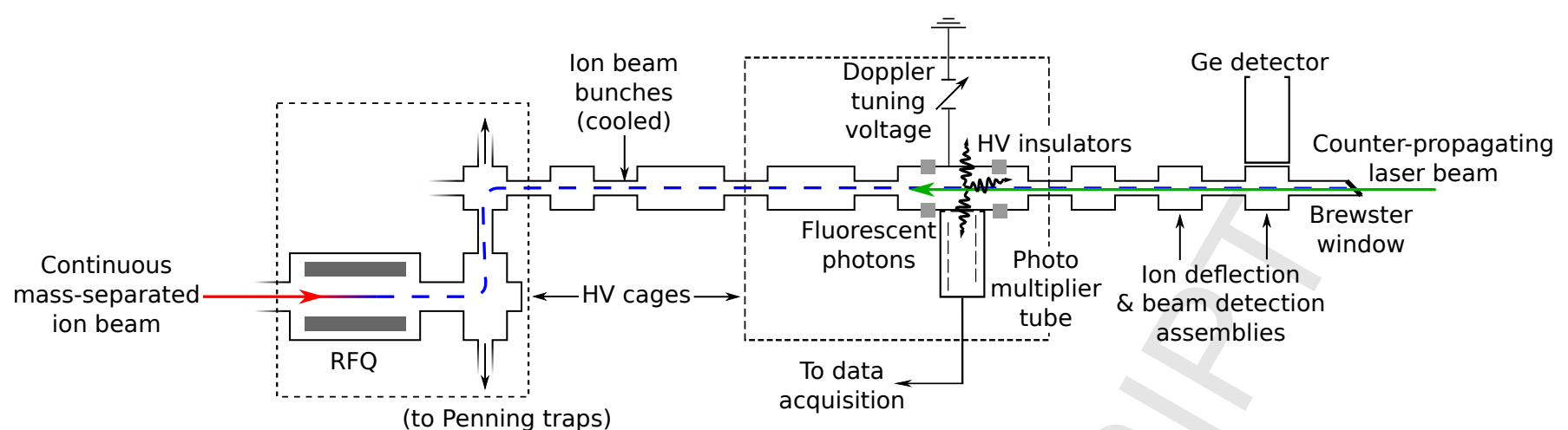

Figure 3: A schematic collinear ion-laser beam line. Ensembles from a radio-frequency quadrupole (RFQ) trap (with ions entering from the left) are overlapped with counter-propagating laser light in a velocity (Doppler) tunable beam-line section. The marked regions indicate voltage isolated sections where ion velocities are manipulated either for stopping (in the RFQ) or for fine adjustment. Beamline components outside of these regions are held at ground potential.

molecules. All but the latter can be reduced by, i), coincidence detection of the fluorescent ion [96] or, ii), bunched delivery of ionic ensembles [127]. Non-optical detection of excitations, those not involving the observation of scattered photons, can be realised by virtue of optical pumping combined with stateselective neutralisation [69] or re-ionisation [35, 36]. Both techniques can be powerfully used to reduce all forms of backgrounds and multiple optical-pumping regions can be applied to ensure optimum sample utilisation.

In all cases the laser frequency in the rest frame of the resonant ion (or atom) may be adjusted by fine tuning the acceleration voltage of the ion beam and "Doppler tuning" the ensembles to resonance. In the case of work with fast atomic beams, where an alkali metal vapour cell is used to neutralise ions to fast atoms, tuning the potential of the vapour cell achieves the final velocity tuning (for ions the potential of the interaction region is adjusted). Different isotopes, by virtue of their different masses, experience differential changes in Doppler shift. This differential change can be advantageous for resolving resonances that overlap in the rest frame.

Important experimental variants of continuous beam collinear spectroscopy, that are in present use, are considered individually below. The variants improve either the precision, efficiency or sensitivity over continuous ion-(single) laser beam spectroscopy and have provided the majority of recent collinear spectroscopic results. Each is exploited at the facilities presented in Section 4.1. One recently productive variant, collinear resonance ionisation spectroscopy, is described later, Section 3.5.2, following a general description of resonance ionisation, Section 3.5.

\subsubsection{Precision collinear spectroscopy}

For typical isotope-shift and hyperfine structure measurements in collinear laser spectroscopy, a precise knowledge of the absolute transition frequency is not required. Only relative frequency separations must be determined with precision limited by statistical uncertainties and systematic limitations on the determination of the high potentials and laser frequencies (and any drifting in these measures). For very light elements however the uncertainties associated with the measurement of the total acceleration voltage determine the overall systematic error which exceeds the perturbation caused by the nuclearvolume effect.

A variant on collinear laser spectroscopy has been realised whereby both counter- and co-propagating lasers were used simultaneously in order to perform the spectroscopy [22]. This technique utilises the 
fact that the product of the resonant frequencies of the co- and counter-propagating laser beams in the laboratory frame is identical to the square of the transition frequency in the rest frame of the ion. The two frequencies are required to be known in absolute terms, achieved to better than $1 \mathrm{MHz}$ with the use of a frequency comb [22]. This technique has been used in the study of the nuclear charge radii of ${ }^{7,9,10} \mathrm{Be}$, the one-neutron halo ${ }^{11} \mathrm{Be}$ and most recently, ${ }^{12} \mathrm{Be}[20,22]$.

\subsubsection{Coincidence detection}

The downstream detection of a fluorescent ion may be used to tag a photon detection to a limit corresponding to the spectrometers ability to determine the position origin of the emitted photon. Use of a segmented photomultiplier and imaging optics can provide a depth-of-field limited coincidence window of $\sim 20 \mathrm{~ns}[96,228]$. The experimental background is solely limited by isobaric contamination of the ion beam causing collisional light or triggering false coincidences. The technique was recently used in the detection of resonance from ${ }^{32} \mathrm{Mg}[30]$.

\subsubsection{Bunched beam spectroscopy}

A powerful and recently popular variant of collinear spectroscopy exploits the ability of on-line, gasfilled, quadrupole traps to efficiently cool and bunch ionic ensembles [127]. The advantages of "bunched" collinear spectroscopy can however already be seen in work of Klempt et al. [229] where a pulsed mode of (reactor) production was exploited to improve the spectroscopic efficiency. In the on-line trap variant, an observation window for fluorescent photons is restricted to a time window during which the bunched ionic ensemble traverses the detection region. Temporal (random) background reductions of $\sim 10^{4}$ may be routinely achieved. The technique can be powerfully enhanced by optically preparing samples in a desired ionic state from which the collinear spectroscopy is then performed.

Example spectra, achieved with bunched beam spectroscopy on ionic yttrium, and with the xordinate transformed from total acceleration voltage to frequency in the rest frame of the ion, are shown in Figure 4 (data taken from ref. [76]). In this application the technique facilitated studies at fluxes of 100 ions $\mathrm{s}^{-1}$.

\subsubsection{Optical pumping}

Optical pumping (the use of light to manipulate atomic state or sub-state populations) features frequently in the spectroscopy of exotic species. Optical pumping has been used,

- to resonantly move population to and from states. The movement of population can be sensitively monitored using state-selective charge exchange or re-ionisation $[35,36,69,75]$ without the need for photon detection (and the associated background and efficiency concerns),

- to induce nuclear orientation in radioactive nuclei, permitting resonance detection on observation of the resulting decay anisotropy. The technique further provides oriented samples for direct nuclear (NMR) spectroscopy (the improvements in experimental sensitivity and precision can be seen, for example, in the work on $\mathrm{Mg}$ reported in the previous section [30]),

- to move populations to states more efficient or better suited to collinear laser spectroscopy [81],

- to sequentially move population to higher excitation energy until ionisation is achieved (next section).

\subsection{Resonance ionisation spectroscopy}

The method of laser resonance ionisation takes advantage of the unique atomic level fingerprint and the high photon absorption cross section on resonance. Resonant photons excite the atom (or potentially ion) either from the ground state or a thermally populated low-lying metastable state using two or 


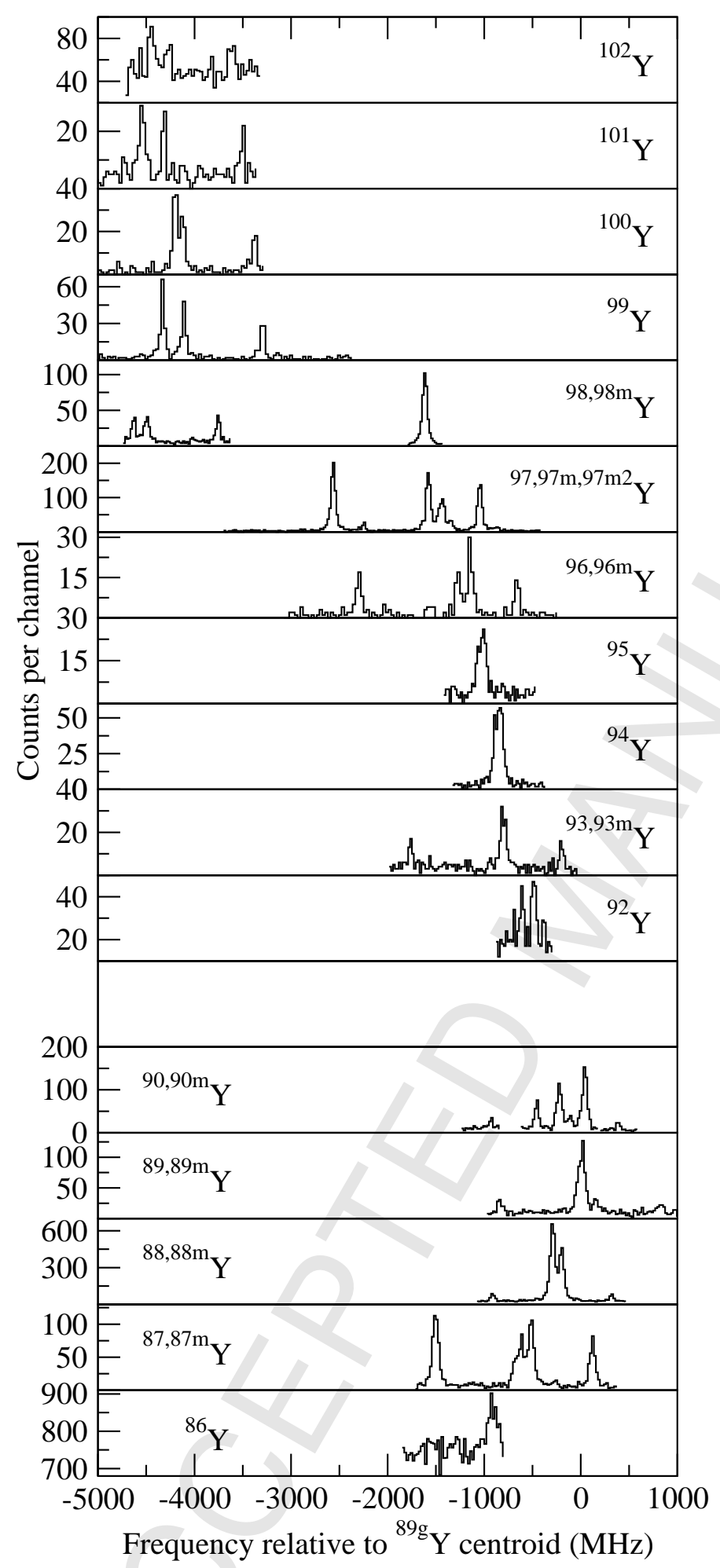

Figure 4: Example yttrium spectra taken with the bunched-beam collinear technique using beam fluxes of $100-3000$ ions s${ }^{-1}$. In the figure the structure of the $363.3 \mathrm{~nm}{ }^{1} \mathrm{~S}_{0}-{ }^{1} \mathrm{P}_{1}$ ionic resonance line is shown for the indicated isotopes and isomers (reproduced from [76]) 
more excitation steps. Ionisation may be achieved directly into the continuum using a final photon, or via high-lying Rydberg states (for subsequent infra-red irradiation, field or collisional ionisation) or via the excitation of an autoionising state. The latter two ionisation mechanisms provide more efficient ionisation of atoms than a simple non-resonant photon. This multi-step process was first demonstrated using rubidium atoms by Ambartsumyan et al in 1971 [230] and in subsequent years was further developed and used in many laboratories. A full theoretical treatment as well as a broad range of applications can be found in the texts of Letokhov [231] and Hurst and Payne [232].

In a simple two-step excitation and ionisation scheme, the flux of photons depopulating the excited state into the continuum, the ionisation rate, must be far greater than any decay into a dark state, $\beta$ $\left(\mathrm{s}^{-1}\right)$,

$$
\sigma_{I} F \gg \beta,
$$

where $\sigma_{I}\left(\mathrm{~cm}^{2}\right)$ is the cross section of ionisation from the excited state and $F\left(\mathrm{~cm}^{-2} \mathrm{~s}^{-1}\right)$ is the photon flux. The fluence condition is defined as

$$
\frac{\sigma_{I} \psi g_{2}}{g_{1}+g_{2}} \gg 1
$$

with $\psi\left(\mathrm{cm}^{-2}\right)$ the fluence of photons and $g_{1,2}$ the statistical weights of the ground- and excited state from which ionisation occurs. These two conditions reflect the requirements on the laser power to achieve saturation and, if met, all atoms irradiated by the laser light will be ionised. Generally, the cross section for a non-resonant ionisation step $\sigma_{I} \sim 10^{-17} \mathrm{~cm}^{2}$ and a typical decay rate $\beta=10^{6} \mathrm{~s}^{-1}$. The flux condition therefore demands that $F \gg 10^{23} \mathrm{~cm}^{-2} \mathrm{~s}^{-1}$. Very often the laser beams will be focused into the interaction region. Assuming a cross-sectional area of $1 \mathrm{~mm}^{2}$, the number of photons required to satisfy the flux condition is much larger than $10^{21} \mathrm{~s}^{-1}$, which is challenging to achieve from a continuous wave (CW) laser system, equivalent to several hundred watts. Pulsed laser systems, with a typical pulse duration of tens of ns, can however readily deliver the required few $\mu \mathrm{J} / \mathrm{pulse}$. For a given intensity, the fluence condition depends on the interaction time of the laser with the atom, or in the case of a pulsed laser system, on the number of laser pulses hitting the atom. In typical ISOL-type applications this leads to a laser pulse energy requirement a factor of 100 larger than that set by the flux condition. Still, such modest requirements can be reached with commercially available pulsed lasers and several on-line facilities utilise solid state pump lasers, copper vapour lasers or excimer lasers for the non-resonant ionisation step if a resonant process is unavailable. These two conditions become more relaxed if the ionization step proceeds resonantly as $\sigma_{I}$ is typically a factor of 100 larger [233].

An outstanding feature of laser resonance ionisation is the high degree of selectivity obtained as a result of the resonant laser-atom interaction. The difference in atomic structure, isotope shifts and hyperfine structures provide the elemental selectivity. If narrow-band lasers are utilised, it may also be possible to discriminate between nuclear ground and isomeric states due to the differences in spins, magnetic dipole and electric quadrupole moments. The selectivity $s$ of a single optical excitation is defined here as the probability of exciting the selected isotope over the probability of exciting a neighbouring isotope. A simple quantitative estimate of the selectivity may be given as

$$
s \sim 4 \cdot(\Delta / \Gamma)^{2} \text { when } \Delta \gg \Gamma .
$$

Here, $\Delta$ is the frequency difference between neighbouring elements, isotopes or isomers, and $\Gamma$ is the interaction linewidth. Although a simple Lorentzian profile is assumed in this model, more realistic lineshapes lead only to minor corrections. As an example, in the resonant $5 \mathrm{~s}^{2} S_{1 / 2} \rightarrow 5 \mathrm{p}^{2} P_{3 / 2}$ transition in silver the isotope shift $\Delta \nu^{107,109} \sim 500 \mathrm{MHz}$ and the natural linewidth $\Gamma \sim 20 \mathrm{MHz}$. Under the assumption of no line broadening nor non-resonant excitation effects, the selectivity $s \sim 2500$.

In a multi-step excitation process the overall selectivity $S$ is the product of the selectivity of each step. Moreover, the isotopic selectivity can be further enhanced with the additional coupling to mass spectrometry. The high selectivity combined with improved sensitivity through the efficient detection of 
ions compared to resonantly scattered photons has resulted in modern laser-based techniques becoming a tool of choice for the study of rare isotopes and ultra-sensitive detection of single atoms, the latter comparable to more mature methods such as accelerator mass spectrometry and low-level counting [234].

The method of RIS for the study of radioactive isotopes was first demonstrated at the ISOL facility, IRIS (Investigation of Radioactive Isotopes on Synchrocyclotron), Gatchina, Russia on the long-lived rare earth isotopes ${ }^{145-149} \mathrm{Eu}[235]$. Following irradiation of a Ta target with a 1-GeV proton beam, reaction products were evaporated and mass separated. Sample sizes of order $10^{10}-10^{12}$ atoms were accumulated onto Ta foils and transferred into a high-temperature atomic beam oven. Resonance ionisation spectroscopy was performed on the atomic beam in a perpendicular geometry to reduce the effects of Doppler broadening. The bandwidth of the pulsed laser which is scanned over the isotope shifts and hyperfine structure is often adjusted to the Doppler width of the atomic ensemble. This leads to a compromise between attaining improved resolution at the expense of lower efficiency, the latter attributed partly to the collimation of the atomic beam and to the duty cycle between laser pulses and continuous atom flux. With a laser linewidth of $600 \mathrm{MHz}$, Alkhazov and colleagues could reach an accuracy of $70 \mathrm{MHz}$ in the isotope shift measurements.

To access shorter-lived Eu isotopes Fedoseyev et al stopped the mass-separated beam directly in a Ta tube, $5 \mathrm{~mm}$ in diameter and $50 \mathrm{~mm}$ long, from which isotopes could be immediately evaporated at temperatures of up to $1500^{\circ} \mathrm{C}$, collimated and ionised [236]. A multi-pass laser interaction region improved the detection sensitivity by an order of magnitude and an efficiency of $3 \times 10^{-4}$ was reached. In a later study of ${ }^{157-172} \mathrm{Tm}$, Mishin et al substantially improved the spectral resolution of the laser used in the transition most sensitive to the hyperfine interaction [237]. A tunable single-frequency ring dye laser was amplified by a pulsed dye laser resulting in a spectral width determined by the pulse length of the pump laser, $\sim 25 \mathrm{MHz}$.

Pulsed ion production can be used in combination with a time-of-flight mass spectrometer to obtain mass identification and to discriminate the radionuclide from lighter molecular ions often created by the high laser power necessary for ionisation. The first demonstration of on-line resonance ionisation spectroscopy coupled with mass identification (RIMS) was performed at the ISOLDE facility for the determination of isotope shifts and hyperfine structure of ${ }^{185-189} \mathrm{Au}$ [238]. The mass-separated ion beam was focused into an atomic beam oven, similar to the method of Fedoseyev, however, the Au isotopes were obtained as decay daughter products of $\mathrm{Hg}$ isotopes. Although a time-of-flight spectrometer improved the signal-to-background ratio (with a mass resolution of $\leq 300$ ), the overall detection efficiency of the RIMS technique was low, $\sim 10^{-8}$, limited due to the $10 \mathrm{~Hz}$ repetition rate of the laser system.

To improve the efficiency of RIS using such low duty cycles, the pulsed-laser induced desorption (PLID) technique was developed and applied to spectroscopy of ${ }^{195} \mathrm{Au}$ [239]. By evaporating radioisotopes using a $10 \mathrm{~ns}$ pulsed laser, a thermal atomic beam can be produced with a temporal profile suitable for efficient resonance ionisation. In resonance, one ion was detected per $10^{5}$ atoms implanted, an improvement by three orders of magnitude compared to the continuous atom beam. Parallel developments were made by groups at McGill University, Montréal, Canada, and Institut de Physique Nucléaire d'Orsay (IPN, Orsay, France), and were used in the study of isotopic chains of Au and Pt at the ISOCELE separator at Orsay [240-242]. A programme of measurements was later initiated at ISOLDE using the COMPLIS (COllaboration for spectroscopy Measurements using a Pulsed Laser Ion Source) setup [139]. The aim of the collaboration was to complement collinear laser spectroscopy studies with high-resolution RIS on elements not easily accessible with the ISOL thick target approach. Rare isotopes of refractory elements with low vapour pressures will decay before extraction because of the long release time. Neutron-deficient isotopes of $\mathrm{Au}, \mathrm{Pt}$ and Ir were studied at COMPLIS as daughter atoms of parent $\mathrm{Hg}$ ions readily produced at IsOLDE. The $\mathrm{Hg}$ ions were extracted, accelerated to $60 \mathrm{keV}$, mass separated, decelerated to $1 \mathrm{kV}$ and implanted into the first atomic layers of a graphite catcher. Following a suitable delay time to optimise production of the daughter nuclei in the graphite, the atoms 
are desorbed using a pulsed laser, resonantly ionised, accelerated and guided to a microchannel plate detector. Most recently, charge radii and nuclear moments have been extracted for several neutron rich isotopes of Sn [91] and Te [94], both produced via fission reactions in the IsOLDE $\mathrm{UC}_{2}$ target. For Sn, the COMPLIS apparatus achieved an efficiency of $\sim 10^{-6}$ and a resolution of $170 \mathrm{MHz}$.

A possible alternative to the conventional graphite catcher used in the RIS-PLID technique has been proposed by Billowes at the University of Manchester [243]. Sample atoms of Bi were implanted into a solid layer of Xe formed on a $\mathrm{Cu}$ substrate connected to a $12 \mathrm{~K}$ cold head of a He cryopump. The atoms were stored for up to 24 hours with no signs of physical or chemical deterioration and were slowly released by warming the catcher substrate to $\sim 80 \mathrm{~K}$. Advantages of the technique include a simpler thermal release of the sample, better spatial and temporal overlap with the multi-step RIS lasers, and a reduced Doppler broadening of the spectral profile. Applications have been proposed to study daughter isotopes produced in the alpha decay of heavy elements.

\subsubsection{Hot cavity in-source resonance ionisation spectroscopy}

A particularly efficient method of laser spectroscopy of radioisotopes was first demonstrated on-line by Alkhazov et al by using laser ionisation in a mass separator ion source [244]. Isotope shifts and hyperfine structures for ${ }^{154-156} \mathrm{Yb}$ were obtained at a production rate of approximately 100 atoms/s for the isotope under study. It was suggested that with improvements in the geometry and by increasing the measurement time it would be possible to decrease the limit of sensitivity to a few atoms per second. Nowadays this technique is commonly known as 'in-source' spectroscopy, whereby the mass-separated ion yield is measured as a function of the wavelength of the pulsed laser used in the production of ions at the source. In-source spectroscopy is a natural development of the hot cavity laser ion source, originally applied at ISOL facilities to increase the ionisation efficiency of atoms moving through vacuum [245-247]. A cavity (typically a tube of refractory material such as W, Ta or Re, or graphite) provides confinement of atoms which, when interrogated with multiple laser frequencies spatially overlapped into the tube, provides an enhancement in the probability of photoionisation. The diffusion time of the atom out of a hot cavity ion source depends on the source dimensions and the thermal velocity of the atoms, thereby determining the minimum repetition rate of the pulsed lasers to maintain the maximum temporal overlap. A thermal ion source operating at a typical temperature of $2000 \mathrm{~K}$ results in a transit time of $\sim 0.1 \mathrm{~ms}$, thus a pulse repetition rate of $10 \mathrm{kHz}$ or more is required. A recent overview of this field, as well as a summary of the ionisation schemes applied across the periodic table both for radioactive ion beam production as well as RIS, can be found in the review of Fedosseev et al [214].

Atoms within a high temperature cavity environment have a broad thermal velocity distribution and thus in-source laser spectroscopy is limited by the significant Doppler broadening of the atomic spectral lines,

$$
\Delta \nu_{D}=7.16 \times 10^{-7} \nu_{0} \sqrt{T / M}
$$

where $T$ is the temperature of the cavity in $\mathrm{K}, M$ the atomic mass number and $\nu_{0}$ the frequency of the atomic transition in $\mathrm{cm}^{-1}$. Although the high temperature conditions are unfavourable for the study of light elements, by applying narrow-band lasers with linewidths approaching $1 \mathrm{GHz}$, the resolution is often sufficient for isotopes of heavy elements that exhibit large field shifts and large hyperfine structures [248]. In the heavy mass region of the nuclear chart, measurements of isotope shifts and hyperfine structures have been carried out using in-source spectroscopy at the IRIS/PNPI facility on a long chain of neutron-deficient $\mathrm{Tl}$ isotopes in the vicinity of the neutron midshell at $N=104$ [142]. At ISOLDE, similar measurements have been applied to $\mathrm{Pb}$ [145-147, 249], $\mathrm{Bi}$ [88] and Po [151-153]. The impressive sensitivity of the technique is highlighted by a measurement of the short-lived isotope ${ }^{182} \mathrm{~Pb}$ $\left(\tau_{1 / 2}=55 \mathrm{~ms}\right)$, detected with a rate of about 1 ion per second. 


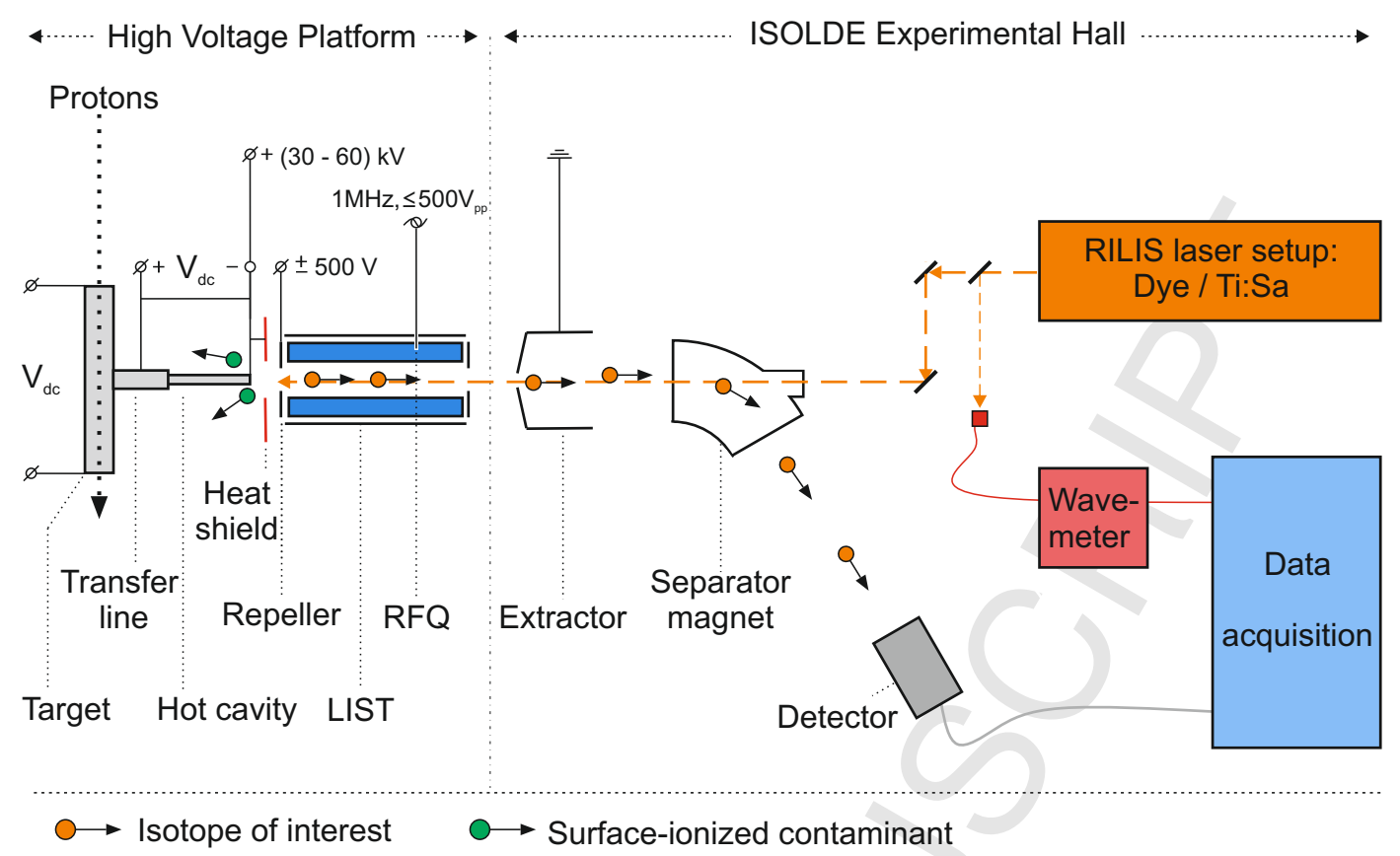

Figure 5: The Laser Ion Source and Trap, LIST, device as installed at the IsOLDE mass separator facility. Following irradiation of the target, reaction products diffuse and effuse into the LIST RFQ where they are laser ionised. Contaminant surface ions are suppressed during LIST operation using a positive voltage on the repeller electrode. Colour on-line. Figure courtesy of D. Fink.

The differences in the hyperfine splitting of atomic lines can also be utilised in the separation of nuclear isomeric states, a possibility first proposed in 1973 by Letokhov [250] and implemented for the first time 25 years later at IsOLDE RILIS for the study of Ag isotopes [251, 252]. Fedosseev et al [214] summarise the isomer-selective ionisation activities demonstrated at IRIS/PNPI, ISOLDE and TRIUMF, often connected with decay studies and/or mass measurements to facilitate a deeper understanding of nuclear structure.

The most recent application of in-source laser spectroscopy has been realised in combination with the LIST (Laser Ion Source and Trap). This device was originally proposed as a means of improving the beam quality from a hot-cavity ion source by decoupling the production and evaporation regions from the ionisation volume, thus addressing the problem of an often poor selectivity due to the isobaric contaminants from surface ionisation [221]. In collaboration with the University of Mainz, the LIST device has been developed for the ISOLDE RILIS [222] while parallel developments have been pursued at the TRIUmF TRILIS facility [253]. The basic operational principle of the IsOLDE LIST is highlighted in Figure 5. A positively charged repeller electrode placed immediately after the exit orifice of the hot cavity suppresses all ions created upstream of the electrode. Neutral atoms diffuse into the laser interaction region of a radio-frequency quadrupole (RFQ) ion guide in which background-free laser ionisation takes place. The photo-ions are confined by the trapping potential of the RFQ as they drift towards the extraction electrode for subsequent acceleration. The polarity and setting of the repeller voltage allows the LIST to be operated either in an ion-guide mode which may be considered normal RILIS operation, or LIST mode. Extensive feasibility studies both off-line and on-line have been performed and a surface-ion suppression factor of up to four orders of magnitude has been achieved. Although the corresponding LIST ionization efficiency was reduced by a factor of 20 in comparison to the ion-guide mode, the impressive selectivity more than compensates for this reduction [222]. 


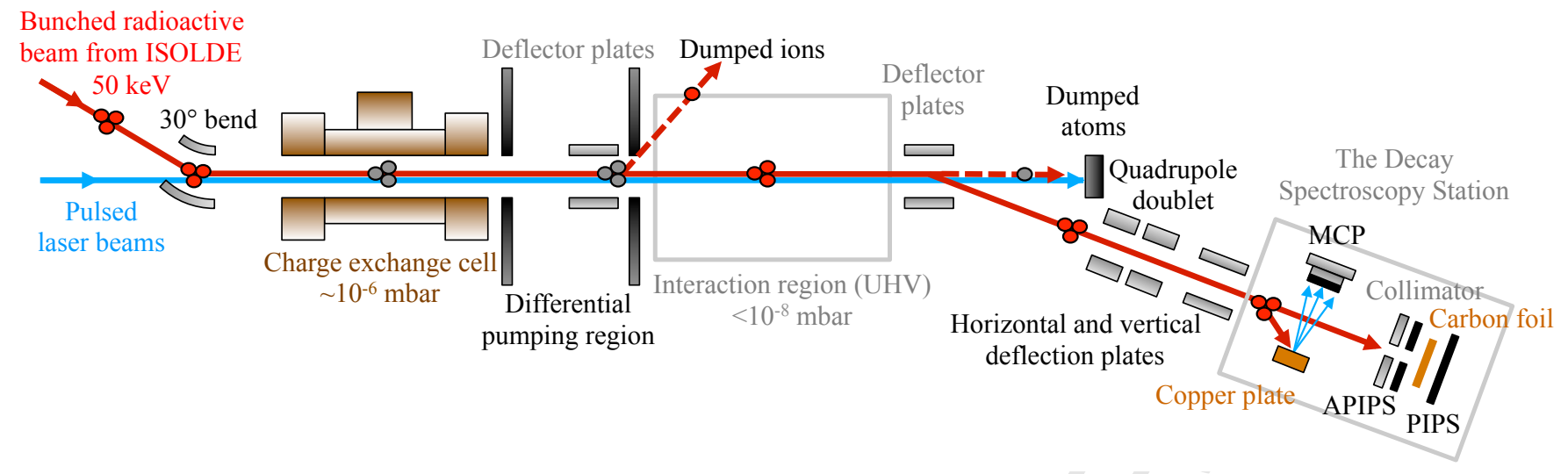

Figure 6: The collinear resonance ionisation spectroscopy beam line at IsOLDE. Photo-ions can either be deflected after the interaction region towards a $\mathrm{Cu}$ plate, releasing secondary electrons which are detected by the MCP, or implanted into a $\mathrm{C}$ foil for charged-particle decay spectroscopy. Colour on-line. Figure courtesy of K. Lynch.

\subsubsection{Collinear resonance ionisation spectroscopy}

The method of collinear-beam resonance ionisation spectroscopy (CRIS) was first proposed more than 30 years ago for ultra-low abundance detection of rare radioactive isotopes [254], and was demonstrated on-line at ISOLDE by Schulz et al on fast atomic beams of radioactive Yb isotopes [119]. The resonance ionisation scheme proceeded from a metastable atomic level populated following neutralisation of the fast ion beam in a charge exchange cell. Isotope shift measurements were performed with typical uncertainties of 5-10 MHz. The limiting factor in this pioneering experiment was primarily associated with the duty-cycle losses associated with the laser-atom interaction. An interaction time of $3 \mu$ s between a continuous atomic beam and the $10 \mathrm{kHz}$ pulsed repetition rate of the laser system resulted in a duty cycle of only $3 \%$. Combined with a low population of the metastable state, a detection efficiency of $1.5 \times 10^{-5}$ ions per beam particle was achieved with the background count rate determined by collisional ionization in the ultra-high vacuum of the ionization region.

The development and installation of gas-filled radio frequency quadrupole cooler devices led to new opportunities for the development of efficient CRIS. The capability to accumulate and deliver bunched radioactive ion beams enables a $100 \%$ temporal overlap with synchronised low-repetition rate pulsed lasers. Soon after the commissioning of the cooler-buncher at the IGISOL facility [255], an off-line test demonstrated an experimental efficiency of 1 detected ${ }^{27} \mathrm{Al}$ ion per 30 atoms, an improvement of more than three orders of magnitude compared to resonance fluorescence detection of ${ }^{27} \mathrm{Al}[256,257]$.

A dedicated beam line for CRIS has now been installed and commissioned at IsOLDE, profiting from the availability of bunched beams from the ISCOOL cooler-buncher [258, 259]. A schematic diagram of the CRIS beam line is shown in Figure 6 illustrating the main principles of the technique. ISCOOL delivers a bunched ion beam with a temporal width between 1-6 $\mu$ s which is deflected into the CRIS beam line and neutralised in-flight in a potassium-vapour charge-exchange cell (CEC). In order to reduce the non-resonant collisional ionisation process, which severely hampered the earlier Jyväskylä work, a differential pumping region separates the CEC from the laser-atom interaction region. Any non-neutral fraction can be deflected within this section. The interaction region is sufficiently long to fully contain the spatial extent of the atom bunch. The arrival of the neutral bunch is synchronised to the pulsed laser beams used in the resonant excitation and ionisation process. Resonantly produced ions are deflected towards a charged-particle detector after leaving the interaction region. In a recent experimental campaign, isotope shifts and magnetic moments of neutron-deficient Fr isotopes were 
measured with half-lives as short as $300 \mathrm{~ms}$ and production rates below 100 atoms/s [158]. A total experimental efficiency of $1 \%$ has been demonstrated which includes transport from the rf coolerbuncher, neutralization, laser ionization and detection. By maintaining a background pressure of $8 \times 10^{-9}$ mbar in the interaction region, non-resonant and collisional ionization was maintained below one ion in $10^{5}$ beam particles.

The technique of decay-assisted laser spectroscopy has recently been developed at the CRIS beam line at ISOLDE [260]. A decay spectroscopy station (DSS) has been mounted at the end of the beam line downstream from the charged-particle detector (Figure 6) and consists of a rotating wheel C foil implantation system and passivated implanted planar Si (PIPS) detectors for charged-particle detection (decay products including alpha, electron and fission fragments). Gamma rays emitted from decay products may be detected by up to three high-purity germanium detectors placed around the implantation site [261]. The first application of the technique demonstrated the use of the DSS to identify components (resolved and unresolved) in the hyperfine spectra associated with low-lying nuclear states in neutrondeficient ${ }^{202,204} \mathrm{Fr}$ by exploiting their characteristic radioactive decay mechanisms [159]. An alpha-decay measurement as a function of laser frequency led to the determination of nuclear observables from the three low-lying isomeric states and extraction of the branching ratios in the decay of ${ }^{204 m 2} \mathrm{Fr}$.

\subsection{Atom and ion traps}

\subsubsection{Magneto-optical atom traps}

The workhorse extensively used in the field of trapped radioactive atoms is the magneto-optical trap (MOT). For the purpose of this review, we briefly present an overview of the technique whose highly elemental, isotopic and isomeric selectivity has, at radioactive beam facilities, been used for charge radii determination in light isotopes, ${ }^{6,8} \mathrm{He}$, as well as in heavy isotopes such as francium (in order to probe the spatial distribution of the nuclear magnetization, the Bohr-Weisskopf effect). Extensive efforts by a number of groups are currently applying modern laser cooling and trapping techniques for precision tests of the Standard Model and for further details we refer the reader to the topical review of Behr and Gwinner [262].

The working principle of the MOT relies on atom confinement via a combination of laser beams from six directions with a weak linear quadrupole magnetic field produced using a pair of anti-Helmholtz coils surrounding the trap centre $[263,264]$. The laser light, circularly polarized, is frequency detuned by a few natural linewidths towards the red of the atomic resonance. Irrespective of motion in any direction the atoms thus see light Doppler shifted towards resonance, absorb these photons and in this manner are cooled and slowed down. The quadrupole field produced by the coils, of order $10 \mathrm{G} \mathrm{cm}^{-1}$, introduces a Zeeman splitting which changes sign at the origin. The combination of the field with the opposite handedness of the retro-reflected beams provides a linear position-dependent restoring force which confines the atoms to the centre. Ignoring effects due to radioactive decay, the lifetime of the cold atom cloud, confined to the centre of the trap $\sim 1 \mathrm{~mm}$ in diameter, is limited by collisions with residual background atoms. For a MOT pressure of $10^{-8}$ Torr a lifetime of $\sim 1 \mathrm{~s}$ may be achieved, sufficient for high precision spectroscopy.

Experimental realisation of a MOT can be demanding, owing to the number of laser frequencies that must be generated for efficient trapping. The technique has been used to the greatest effect on elements with rather simple electronic structures including alkali, alkali-earth and noble gases [262]. In order to cool atoms from room temperature efficient cycling transitions which transfer population back and forth between the ground and excited states are required. Efficient cycling demands that all potential "dark" states, those in which populations would experience no further laser interaction, must be re-pumped and recovered. Radioactive isotopes of almost all alkali elements ( $\mathrm{Na}, \mathrm{K}, \mathrm{Rb}, \mathrm{Cs}, \mathrm{Fr}$ ) have been trapped and, with additional lasers used to repump lost population to metastable states, all 


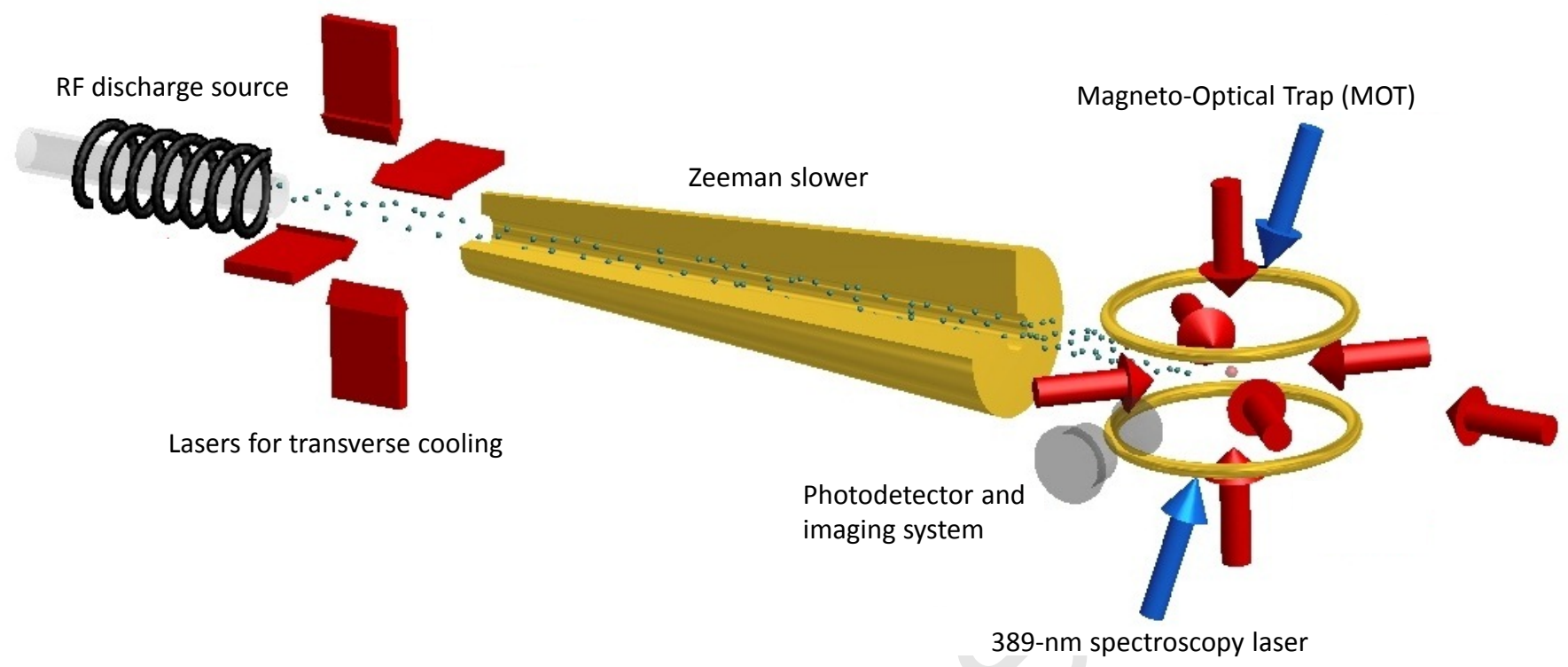

Figure 7: Schematic of the apparatus used to cool and trap ${ }^{6,8} \mathrm{He}$ atoms. Following excitation to the $2{ }^{3} S_{1}$ metastable level in an RF gas discharge source, the atoms are collimated via transverse cooling, decelerated and captured in a magneto-optical trap (MOT). Detection and spectroscopy was performed, imaging the fluorescence light onto a photomultiplier tube. Colour on-line. Figure courtesy of P. Mueller.

stable alkaline-earth elements and Rn have also been trapped. It is currently not possible to trap noble gas atoms using ground state transitions, however single-electron cycling transitions are accessible from metastable states. Prior to laser cooling and trapping this requires population of the metastable state by some other method, typically DC- or RF-induced discharge.

The selective cooling and trapping of helium atoms in a MOT with single-atom sensitivity, high signal-to-noise ratio and excellent spectroscopic resolution allowed the neutron-rich halo nuclei ${ }^{6} \mathrm{He}$ and ${ }^{8} \mathrm{He}$ to be probed, completely free from any contamination by the dominant ${ }^{4} \mathrm{He}$ isotope. The technique was developed and first applied to laser spectroscopy of ${ }^{6} \mathrm{He}$ at Argonne National Laboratory, USA [13], prior to a move of the apparatus to GANIL, France, where the first measurement was performed on ${ }^{8} \mathrm{He}$ as well as an improved measurement on ${ }^{6} \mathrm{He}$ [14]. Figure 7 provides a schematic of the apparatus used in the experiments. The atoms were prepared in the $1 \mathrm{~s} 2 \mathrm{~s}{ }^{3} S_{1}$ metastable state by passing a thermal He beam through a radio-frequency discharge cell, resulting in a fraction $\left(10^{-5}\right)$ excited to the $2{ }^{3} S_{1}$ metastable level. After being transversely cooled and decelerated using a Zeeman slower the ortho-helium was captured and trapped using the $1 \mathrm{~s} 2 \mathrm{~s}{ }^{3} S_{1} \rightarrow 1 \mathrm{~s} 2 \mathrm{p}{ }^{3} P_{2}$ transition at $1083 \mathrm{~nm}$.

Whilst the efficiency of trapping a He atom from production is approximately $10^{-7}$, once trapped the atoms are at a temperature of a few $\mathrm{mK}$ and can be probed for their entire lifetime (170 ms in the case of ${ }^{8} \mathrm{He}$ ) allowing many thousands of photons to be scattered per second. Resonance fluorescence spectroscopy was performed on the $1 \mathrm{~s} 2 \mathrm{~s}{ }^{3} S_{1} \rightarrow 1 \mathrm{~s} 3 \mathrm{p}{ }^{3} P_{J}$ transitions at $389 \mathrm{~nm}$, the scattered photons imaged onto a photomultiplier tube. A capture rate of $\sim 20000{ }^{6} \mathrm{He}$ atoms per hour was achieved, with a corresponding 30 per hour for ${ }^{8} \mathrm{He}$. It was possible to measure the isotope shifts of ${ }^{6,8} \mathrm{He}$ with sub-100 $\mathrm{kHz}$ precision [14].

One of the heaviest elements trapped to date is francium $(Z=87)$. With a simple electronic structure, the heaviest alkali element has been the focus of several studies since the 1990s in the context of atomic parity non-conservation (APNC) experiments which search for new physics beyond the Standard Model 
[262]. Francium exhibits a number of attractive features essential for understanding atom-nucleus interactions as well as weak interactions. Firstly, the effect of APNC scales with $Z^{3}$, second, the simple atomic structure allows the application of ab initio calculations of its properties which can be experimentally tested and third, a large number of isotopes are accessible at appropriate on-line facilities. In order to interpret parity-violating anapole moments it is important to understand limitations due to nuclear structure effects, for example the nuclear magnetization. This may be done through a systematic study of the hyperfine structure across a chain of isotopes.

The FrPNC collaboration at the ISAC facility, TRIUMF, is currently proceeding with measurements in Fr having recently commissioned the Francium Trapping Facility [265]. A $500 \mathrm{MeV}$ proton beam from the TRIUMF cyclotron interacting with a hot $\mathrm{UC}_{x}$ target can produce a number of neutron rich and neutron deficient isotopes at rates of $10^{7}$ to $10^{9}$ per second. The activity is first deposited in a Y foil which acts as a neutralizer before being heated, releasing neutral Fr atoms into a specially coated glass cell (the capture chamber). In this cell a few $10^{5}$ atoms are captured within a MOT in preparation for Doppler-free spectroscopy. The MOT is formed by two lasers, a trapping and repump laser operating at $718 \mathrm{~nm}$ driving the cycling $D_{2}$ line which leaves a third laser (probe) for spectroscopy using the $D_{1}$ line at $817 \mathrm{~nm}$. Fast radio-frequency (rf) scans of sidebands added to the probe laser are used for isotope shift measurements [161] as well as obtaining hyperfine splittings, the latter measured to 100 ppm precision which is sufficient to investigate the hyperfine anomaly [266]. In the future the cold francium sample will be transferred from the MOT to an ultra-high vacuum science chamber which will be used for the study of APNC.

\subsubsection{Ion traps}

Ion traps, notably (but not exclusively) the Paul trap [267] play a critical role in a number of the spectroscopic studies reviewed here.

A classical Paul, or quadrupole ion trap, may be constructed from opposite facing hyperbolic electrodes and a central (hyperbolic) ring electrode. Counter-oscillating AC potentials (typically at radiofrequencies) and static DC potentials applied to the electrodes act to dynamically confine ions. In the majority of the reviewed work a "linear", often segmented, variant of the trap is constructed from four parallel electrodes with static DC fields providing containment in the axial direction. Gas-filled linear Paul traps form the basis of the on-line cooler-bunchers exploited in the spectroscopy in Section 3.4.3. Low pressures, $\leq 1 \mathrm{mbar}$, of hydrogen or helium in such traps act to damp the ion motion (of species heavier than the buffer gas) and can cool trapped ensembles to sub-eV energies [127]. Cooling to $\mu \mathrm{eV}$ energies can be achieved using laser cooling in the same manner as in atomic trapping described in the preceding section - counter-propagating redshifted resonant laser light driving against motion in the direction of the laser.

With respect to the spectroscopy of radioactive nuclei both spectroscopy within traps and on species ejected from them play a critical role in modern studies. An early use of a gas-filled classical Paul trap [170] succeeded in studying Th isotopes from $A=227-230$ and 232. Observation of resonant scatter from a single laser field at wavelengths longer than that of the laser light permitted efficient filtering of randomly scattered light and the cooling buffer gas could be used to quench the metastable level populated in the spectroscopy (recovering the ions for further excitation). Significant Doppler broadening associated with the $(\sim \mathrm{eV})$ trap motion was observed but substantially reduced by a 2-stage, stepwise laser excitation (with counter-propagating beams and sample velocity selection) permitting hyperfine structure measurements.

Recent high-resolution spectroscopy of thorium has been achieved in the triply charged, monovalent, ion for ${ }^{229} \mathrm{Th}$ and ${ }^{232} \mathrm{Th}$ [167]. The spectroscopic approach used has many features in common with independent work that has achieved laser microwave double resonance (LMDR) spectroscopy of singlycharged ${ }^{7} \mathrm{Be}$ and ${ }^{11} \mathrm{Be}[21,23]$. In both radioactive ions are injected into a linear Paul trap in which 
buffer gas is originally present. Following loading the buffer gas is rapidly pumped away and laser cooling applied (in two separate, sequential, stages in the case of $\mathrm{Th}^{3+}$ ). Crystallisation (the formation of an ion crystal [268]) is achieved and high-resolution spectroscopy of the trapped ensembles performed.

In the Be studies a (weak) $0.25-1.5 \mathrm{mT}$ magnetic field parallel to the laser radiation permitted LMDR spectroscopy precisely measuring (Zeeman split) transitions in the ionic ground state. For ${ }^{11} \mathrm{Be}$ a production of $10^{6}$ ions per second resulted in 70 per second being extracted from the gas cell (see next section) and, after a 40 second loading, $\sim 110$ cooled ions in the trap. In the thorium work the $\mathrm{Th}^{3+}$ was produced by laser ablation of bulk sample. A simultaneous loading of ${ }^{229} \mathrm{Th}$ and ${ }^{232} \mathrm{Th}$ was observed to achieve an ultra-cold phase by sympathetic cooling (only one isotope was cooled) and to be stable for "a large fraction of an hour" [167].

Beyond Paul traps, ion, including single ion, spectroscopy has been demonstrated for stable species in magnetic and electrostatic traps. Spectroscopy of radioactive species in Penning traps, such as SpecTrap [269], and electrostatic multi-reflection traps, such as the ConeTrap [270, 271], are returned to and discussed in Sections 4.1 and 8.

\subsection{Gas cells and ion guides}

The stopping and thermalisation of high-energy nuclear reaction products can be readily achieved in solid material. Subsequent release of the species on heating can be efficiently attained for many chemical elements from refractory element stoppers, such as W, Ta, Re and C, at elevated temperatures around $2000 \mathrm{~K}$ (and these materials form the basis of the atom and ion sources described in Section 3.3). The release times, which can be of order seconds, and inability to extract native refractory species led to historical efforts to explore chemically assisted, volatile molecular release (fluorination of the ion source) and the use of high purity $(\sim \mathrm{ppb})$ gas catchers. Fluorescence spectroscopy directly within gas cells has been achieved on-line for radioactive Yb isotopes [118] (and off-line, using collected samples, for radioactive $\mathrm{Bi}$ isotopes [148-150]). A far greater range of isotopes have been studied on-line using ions extracted from gas cells, either gas stoppers or ion guides.

In an ion guide source a fast-flowing helium gas jet is released from a reaction chamber that holds a pressure of $\sim 250$ mbar of He gas [272]. Nuclear reaction products, including fission fragments, are produced from beam-target nuclear reactions in the chamber and are evacuated in the jet on a timescale of order 1 millisecond. The rapid evacuation ensures a substantial fraction of the nuclear recoils (up to a few percent) remain charged on exiting the chamber where they are isolated from the buffer gas in a secondary sextupole RF ion guide (SPIG). At the exit of the SPIG the charged species, dominated by singly-charged ions, are injected into a high vacuum $\left(10^{-6} \mathrm{mbar}\right)$ region for acceleration (typically to $30 \mathrm{keV}$ ). The production technique shows great insensitivity to the chemical nature of the nuclear recoil and has been particularly exploited in the study of elements in refractory regions (with optical studies concentrating on collinear spectroscopy of elements $\mathrm{Y}-\mathrm{Tc}$ ).

Efficient spectroscopy using ion guides was achieved with the development of the on-line coolerbuncher and pioneering work on the laser spectroscopy of bunched beams [127]. Further improvement to the scope of the spectroscopy was achieved with the development of in-cooler optical pumping that facilitates access to chemical systems previously impossible at any collinear facility. In the technique, outlined in Section 3.4.4, atomic populations in radioactive ions are pumped to states optimal for secondary high-resolution (collinear) spectroscopy while the ions are being cooled and bunched in the RFQ. The movement of population can be achieved at wavelengths unreachable for high-resolution lasers (instead being achieved using pulsed broad-bandwidth lasers) but, following the pumping, result in populations from which accessible transitions can be found [81]. Similarly weak transitions from the ionic ground state (prohibitively inefficient for collinear spectroscopy) can be driven for the duration of the bunching time, typical $10-100 \mathrm{~ms}$, two to three orders of magnitude longer than the fast beam interaction time. 
Large scale gas cells, capable of stopping up to $\sim \mathrm{GeV}$ energy recoils, are commonly referred to as gas stoppers and are in use at a number of facilities described in 4.1 (and above). The devices, which can operate up to atmospheric pressure, use radio-frequency structures to prevent diffusion and adhesion to the cell walls while thermalised ions are field drifted to an extraction region (and separated from the gas as in the ion guide technique). The attainable drift velocities, which correspond to $\sim 5$ seconds extraction time from the rear of the chamber in ref. [23], and space-charge saturation limit the efficiency of the devices. A novel solution has been proposed by Bollen [273] and is now under construction [274]. The device, a gas-filled "inverse" cyclotron, uses a cyclotron construction to capture and slow energetic recoils to a central extraction region. Concentric radiofrequency structures at the centre permit efficient extraction and space-charge limitations and release times (expected to be $\sim 5$ milliseconds) are vastly improved.

\subsubsection{In-gas-cell resonance ionisation spectroscopy}

The development of laser ionisation of neutral recoils in an on-line gas cell was first proposed by van Duppen et al [275] and demonstrated at LISOL [219, 220]. In this variation of the ion guide technique pioneered at Jyväskylä [215], the group combined the fast and universal thermalisation of reaction products in a gas, the high selectivity and efficiency of resonant laser ionisation and, overcoming the low-duty cycle problem of high power lasers, the ion (and atom) storage capability of a high-pressure gas cell. In order to encourage the recombination of reaction products, argon buffer gas is often used which favours the presence of only a weakly ionised plasma created by the primary accelerator beam, recoil ions and secondary radioactivity. During the course of many studies, the need for conditions of high purity (sub-parts-per-billion) within the gas cell has been realised [276, 277], in particular for the most chemically active ions which include several refractory elements that cannot be accessed via more traditional ISOL techniques. The complexities associated with addressing the main loss mechanism for photo-ions during evacuation from a gas cell, namely recombination in the presence of buffer gas electrons created by the primary beam, led to the recent development of physically separating the stopping and laser ionisation volumes [278]. Gas flow transports recoils from the stopping chamber to the laser ionisation volume which can be illuminated along the extraction axis or transversely. The latter ionisation geometry, close to the exit hole, allows the use of an upstream ion collector to collect any non-neutral fraction thus dramatically improving the selectivity of the laser ion source.

In a similar drawback to the hot cavity in-source approach, the spectral resolution of an atomic resonance inherently suffers from the environmental effects within the gas cell, namely pressure broadening and shift due to collisions with the buffer gas atoms. With a typical operating pressure of between 200500 mbar, the spectral resolution is thus limited to a few GHz. Nevertheless, in-gas-cell spectroscopy has been successfully performed at the LISOL separator on the neutron-deficient isotopes of $\mathrm{Cu}[60,62]$, $\mathrm{Ag}$ [86] and in off-line studies of ${ }^{209} \mathrm{Bi}$ at the IGISOL facility, Jyväskylä [279]. The average spectral resolution of the LISOL experiments was found to be 4 and $10 \mathrm{GHz}$ for $\mathrm{Cu}$ and $\mathrm{Ag}$, respectively. In a first step towards the very-heavy region of the nuclear chart, a recent campaign of measurements at LISOL has focused on Ac. Preliminary in-gas-cell broadband laser spectroscopy was performed on ${ }^{212} \mathrm{Ac}$, revealing a considerable spectral linewidth of $\sim 30 \mathrm{GHz}$ for the excitation step [280]. High-resolution RIS on long-lived ${ }^{227} \mathrm{Ac}\left(\tau_{1 / 2}=21.8\right.$ years $)$ was performed in collaboration with the University of Mainz and Jyväskylä to identify a more suitable transition for in-gas-cell spectroscopy [281]. In that work, five transitions were investigated and hyperfine structure parameters were determined for the first time. The most promising transition with the largest total splitting is shown in Figure 8. This transition was subsequently used for isotope shift measurements (with respect to atoms of ${ }^{227} \mathrm{Ac}$ evaporated from a heated filament) of ${ }^{212} \mathrm{Ac}$ up to ${ }^{215} \mathrm{Ac}\left(\tau_{1 / 2}=170 \mathrm{~ms}\right)$ at LISOL, the latter located at the $N=126$ shell closure.

Resonance ionisation spectroscopy in an Ar buffer gas cell has also been developed at the University 


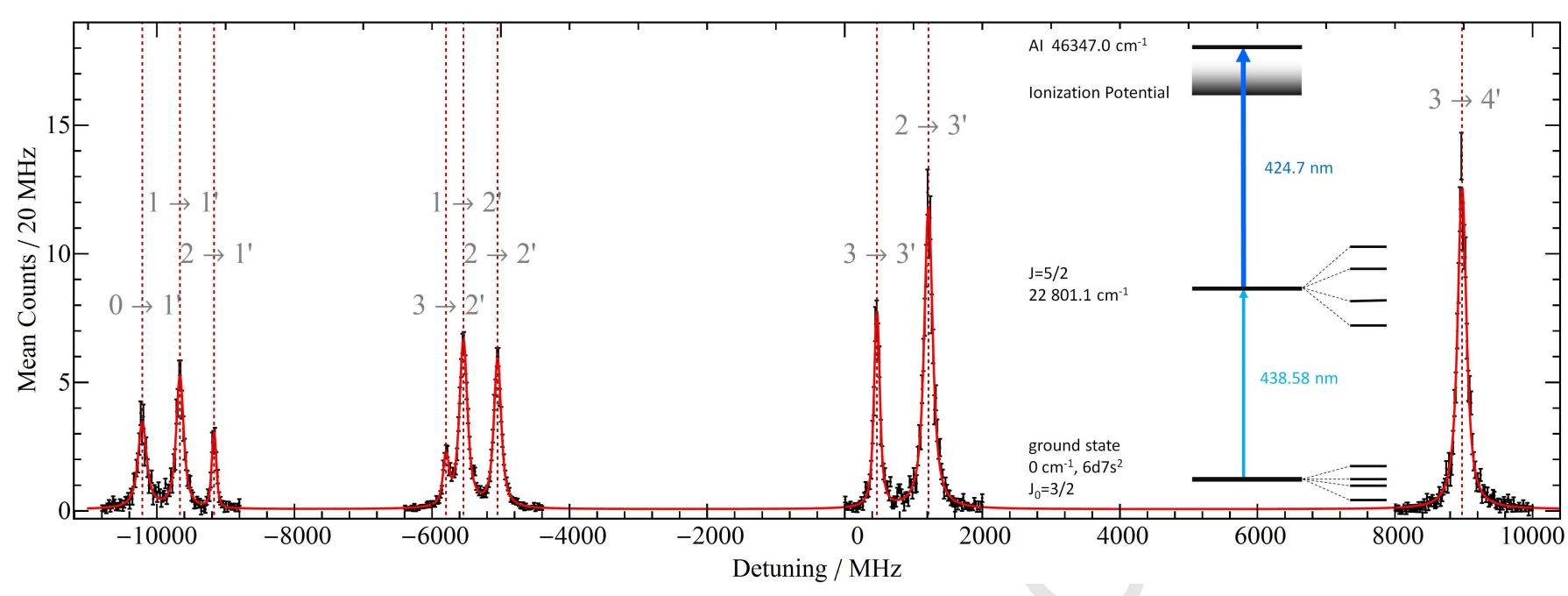

Figure 8: Hyperfine structure of ${ }^{227}$ Ac following high-resolution RIS on the $6 \mathrm{~d} 7 \mathrm{~s}^{2}{ }^{2} D_{3 / 2} \rightarrow 6 \mathrm{~d} 7 \mathrm{~s}(3 \mathrm{D}) 7 \mathrm{p}$ ${ }^{4} P_{5 / 2}$ atomic transition [281]. The dashed vertical lines indicate the centroids of the hyperfine components which are labeled with the $F \rightarrow F^{\prime}$ notation.

of Mainz by Backe and colleagues for the investigation of transuranium nuclides which cannot be produced using spallation reactions [282, 283]. The so-called RADRIS (RAdiation Detected Resonance Ionisation Spectroscopy) method was developed to perform hyperfine spectroscopy of heavy-ion induced reaction products. The separated fusion-evaporation products are stopped in the gas cell at pressures of about 100 mbar. The fraction of ions that are not neutralised can be guided by suitable electric fields to a collection filament or pushed back to the source electrode if operating the system under off-line conditions. The remaining neutral atoms slowly diffuse to the walls of the gas cell in a time scale of $\sim 50 \mathrm{~ms}$, sufficiently long to have multiple interactions with the lasers thus eliminating any duty factor losses, even with modest repetition rates. The photo-ions are then guided by electric fields towards a particle detector where the ion is detected via its radioactive decay. The technique was first developed off-line using the $\beta$-active isotope ${ }^{208} \mathrm{Tl}$, with a sensitivity of $1.3 \times 10^{-3}$ beta counts per ${ }^{208} \mathrm{Tl}$ recoil, limited by the neutralisation fraction in $\operatorname{Ar}(\sim 13 \%)$ and the fraction which diffused into the laser-atom interaction volume [284]. The culmination of the RADRIS experiments were the successful measurements of isotope shifts for superdeformed Am fission isomers ${ }^{240 f, 242 f, 244 f}$ Am [183, 184]. Samples were produced via deuterium-induced fusion-evaporation reactions on ${ }^{242,244} \mathrm{Pu}$ targets at the tandem accelerator, Heidelberg. The low energy recoils required acceleration potentials of up to $95 \mathrm{kV}$ in order to penetrate the window of the gas cell. The sensitivity of the technique was such that spectroscopy could be performed on target production rates as low as $10 \mathrm{~s}^{-1}$ and radioactive nuclides with half-lives as short as $1 \mathrm{~ms}$.

The RADRIS method was later used in several on-line experiments with the lanthanide isotopes ${ }^{152,153} \mathrm{Er}$ and ${ }^{155} \mathrm{Yb}$, the latter a homolog of ${ }^{254} \mathrm{No}$. The first experiments on ${ }^{155} \mathrm{Yb}$ resulted in a rather low detection efficiency, $\sim 10^{-5}$, attributed to a poor overlap between the lasers and the stopping volume, and the rather high fraction $(\sim 85 \%)$ of non-neutralized ions which are simply discarded. This led to the development of the so-called ICARE technique (Ion Collection and Atom Re-Evaporation), in which the non-neutral fraction is transported by electrical fields to a catcher filament, adsorbed and released as atoms using a short current pulse. Spectroscopy is then performed using a two-step excitation scheme to a Rydberg state from which ionization occurs by buffer gas collisions. Following detection by the alpha decay of the resonantly produced ions, an overall efficiency of $0.8 \%$ with respect to the production rate at the target was deduced, a considerable improvement over the first experiments [285]. 
The detection of the ionisation process by radioactive decay limits the applicability of the RADRIS method to nuclides with half-lives of less than a few minutes. In a separate development, radioactive decay detection was replaced by mass-selective direct detection of the ions, in the so-called IGRIS (Ion Guide-detected Resonance Ionisation Spectroscopy) technique [282]. Ions are transported by a suitable electric field to the nozzle of the gas cell, separated from the gas jet and mass analysed with a quadrupole mass spectrometer. Off-line studies on a sample containing $2.7 \times 10^{10}$ atoms of the isotope ${ }^{255} \mathrm{Fm}\left(\tau_{1 / 2}=20.1 \mathrm{~h}\right)$ resulted in the first ever observation of two atomic transitions in Fm $(Z=100)$ [283]. This highlighted the feasibility of atomic spectroscopy of an element with an unknown level scheme and was later followed up by a second experiment which extracted information on the transition rates and hyperfine structure of the two transitions [191]. To date, Fm is the heaviest element to have been successfully addressed by laser spectroscopy.

\subsubsection{In-gas-jet resonance ionisation spectroscopy}

The possibility of using the gas jet environment as an ionisation region was initially explored following the suggestion of coupling the LIST method with the gas cell [286]. In a variation of the hot-cavity LIST, reaction products neutralise within the gas cell and upon exit they are selectively ionised with lasers. The photo-ions are captured by the radio-frequency (rf) field of a SextuPole Ion Guide (SPIG) for further transport to the mass separator. A positive DC voltage applied to the first electrode of the SPIG acts to repel any non-neutral fraction thus ensuring the highest possible beam purity for subsequent experiments. To ensure an efficient ionisation of the reaction products there are two parameters which must be satisfied, namely a good geometrical and temporal laser/atom overlap. A full temporal overlap is guaranteed if the laser repetition rate is high enough to irradiate all atoms in the atomic jet. For example, in a supersonic Ar gas jet with velocity $550 \mathrm{~ms}^{-1}$ then, for a laser repetition rate of $10 \mathrm{kHz}$, an interaction length of $5.5 \mathrm{~cm}$ is required.

Exploratory studies on the impact of the gas jet on the resonant linewidth of $\mathrm{Ni}$ were performed by Sonoda et al at the LISOL facility using the low-repetition rate $(200 \mathrm{~Hz})$ excimer-based laser system [287]. Although the laser bandwidth was $1.6 \mathrm{GHz}$ isotope shifts in stable ${ }^{58,60,62,64} \mathrm{Ni}$ were observed. In addition, laser Doppler-shift velocimetry in a longitudinal ionisation geometry was used to determine the flow velocity of moving atoms within the jet in comparison to atoms in a reference cell. A Ni resonance peak displacement of $\sim 7 \mathrm{GHz}$ in $\mathrm{He}$ and $\sim 2.5 \mathrm{GHz}$ in Ar resulted in jet velocities of $1663 \mathrm{~ms}^{-1}$ and $550 \mathrm{~ms}^{-1}$ for He and Ar, respectively. On-line, a comparison between the low-repetition rate dye laser system and a high-repetition rate Ti:sapphire laser system on ${ }^{59} \mathrm{Cu}\left(\tau_{1 / 2}=81.5 \mathrm{~s}\right)$ demonstrated the significant improvement of the time-overlap efficiency using the Ti:sapphire system while at the same time indicating the need for better spatial overlap with the atomic jet [288].

In order to meet the requirement of a good spatial overlap between the atomic jet and laser beams several off-line studies of the gas flow characteristics have been performed as a function of nozzle type and gas cell-to-background pressure ratio [289]. The de-Laval nozzle is the most popular type of converging-diverging nozzle which generates a supersonic flow of approximately constant temperature and density. If one is able to carefully tailor the divergent part of the nozzle then a uniform flow is created with parallel streamlines, characterised by a single Mach number $(M)$. The gas temperature, density and pressure drop very fast as the Mach number increases. For example, the gas-jet velocity of Ar reaches $99 \%$ of its maximum value $\left(558 \mathrm{~ms}^{-1}\right)$ at $M=12$, at which point the temperature of the jet reaches $6 \mathrm{~K}$ [290]. According to Eq. 27, this corresponds to a reduction in the Doppler broadening from $940 \mathrm{MHz}$ at room temperature to $130 \mathrm{MHz}$ for a 261.241-nm transition in $\mathrm{Th}(A=229)$. In a recent (final) experiment at LISOL, high-resolution in-gas-jet spectroscopy was performed on ${ }^{214,215}$ Ac [291] with a spectral resolution as low as $300 \mathrm{MHz}$, a selectivity of $\sim 200$ and an efficiency of $\sim 0.5 \%$, the latter confirming expectations discussed by Ferrer [280]. 


\subsection{Comparison of techniques}

Table 2 shows, by chemical element, which of the different techniques described in this section have been applied to the study of radioactive isotopes. The total use of the techniques, grouped by general category (collinear beam, crossed beam, RIS, trap, gas cell and "others") are shown in Figure 9. Figure 9 shows how the techniques have been deployed over all measurements and for those highlighted as recent in Table 1.

Table 2: The spectroscopic techniques used in the studies cited in Table 1 . The abbreviations denote techniques described in this chapter:

CB: Collinear Beam (F: Fluorescence, B: Bunched, M: Light Modulation, RIS: Resonance Ionisation, S: State Selective, BNMR: Nuclear Magnetic Resonance, BNQR: Nuclear Quadrupole Resonance, PIC: PhotonIon-Coincidence, CC: Counter- and Co-propagating Laser Beams)

XB: Crossed Beam (F: Fluorescence, TP: Two Photon, RIS: Resonance Ionisation, OCFS: Optical Frequency Comb Sythesizer)

IT: Ion Trap (F: Fluorescence, DR: Double Resonance)

AT: Atom Trap

RIS: Resonance Ionisation (IS: In Source, IG: Ion Guide, GC: Gas Cell)

GC: Gas Cell (F: Fluorescence, OP: Optical Pumping)

SP: Saturation Spectroscopy

SG: Spectrograph

HC: Hollow Cathode

HTC: Hot Cell

LIOP: Laser Induced Optical Pumping

RADOP: Radioactive Detection of Optical Pumping

PLID: Pulsed Laser Induced Desorption

ABMR: Atomic Beam Magnetic Resonance incl. Double

Resonance

2SF: 2 Step Fluorescence

OLC: Optical Level Crossing

\begin{tabular}{lcll}
\hline \hline Element & $Z$ & Recent Techniques & All Techniques \\
\hline \hline $\mathrm{He}$ & 2 & XB(OCFS) & AT, SP, XB(OCFS) \\
$\mathrm{Li}$ & 3 & XB(TP,RIS), CB(BNQR) & ABMR, CB(BNMR), CB(NQR), XB(TP,RIS $)$ \\
$\mathrm{Be}$ & 4 & CB(PIC) & CB(PIC), CB(F,CC), IT(DR) \\
$\mathrm{Ne}$ & 10 & CB(S) & CB(S), LIOP, RADOP \\
$\mathrm{Na}$ & 11 & & ABMR, LIOP, RADOP \\
$\mathrm{Mg}$ & 12 & $\mathrm{CB}(\mathrm{F}), \mathrm{CB}(\mathrm{BNMR})$ & $\mathrm{CB}(\mathrm{F}), \mathrm{CB}(\mathrm{BNMR})$ \\
$\mathrm{Al}$ & 13 & & XB(F) \\
$\mathrm{Si}$ & 14 & RIS(IS,TP) & RIS $(\mathrm{IS}, \mathrm{TP})$ \\
$\mathrm{Ar}$ & 18 & & $\mathrm{CB}(\mathrm{S})$ \\
$\mathrm{K}$ & 19 & $\mathrm{CB}(\mathrm{B}, \mathrm{F}), \mathrm{SP}$ & $\mathrm{AT}, \mathrm{CB}(\mathrm{B}, \mathrm{F}), \mathrm{LIOP}, \mathrm{RADOP}, \mathrm{SP}$ \\
$\mathrm{Ca}$ & 20 & $\mathrm{CB}(\mathrm{B}, \mathrm{F})$ & $\mathrm{CB}(\mathrm{B}, \mathrm{F}), \mathrm{CB}(\mathrm{S}), \mathrm{XB}(\mathrm{F})$ \\
$\mathrm{Sc}$ & 21 & $\mathrm{CB}(\mathrm{B}, \mathrm{F})$ & $\mathrm{CB}(\mathrm{B}, \mathrm{F})$
\end{tabular}




\begin{tabular}{|c|c|c|c|}
\hline $\mathrm{Ti}$ & 22 & & $\mathrm{XB}(\mathrm{F}), \mathrm{CB}(\mathrm{B}, \mathrm{F})$ \\
\hline $\mathrm{Mn}$ & 25 & $\mathrm{CB}(\mathrm{B}, \mathrm{F})$ & $\mathrm{CB}(\mathrm{B}, \mathrm{F}), \mathrm{CB}(\mathrm{F})$ \\
\hline $\mathrm{Fe}$ & 26 & $\mathrm{CB}(\mathrm{F})$ & $\mathrm{CB}(\mathrm{F}), \mathrm{XB}(\mathrm{F})$ \\
\hline $\mathrm{Ni}$ & 28 & & $\mathrm{HC}$ \\
\hline $\mathrm{Cu}$ & 29 & $\mathrm{CB}(\mathrm{B}, \mathrm{F}), \mathrm{RIS}(\mathrm{IS})$ & $\mathrm{CB}(\mathrm{B}, \mathrm{F}), \mathrm{RIS}(\mathrm{IG}), \mathrm{RIS}(\mathrm{IS})$ \\
\hline $\mathrm{Zn}$ & 30 & & $\mathrm{CB}(\mathrm{F})$ \\
\hline $\mathrm{Ga}$ & 31 & $\mathrm{CB}(\mathrm{B}, \mathrm{F})$ & $\mathrm{CB}(\mathrm{B}, \mathrm{F})$ \\
\hline $\mathrm{Kr}$ & 36 & & $\mathrm{CB}(\mathrm{S})$ \\
\hline $\mathrm{Rb}$ & 37 & $\mathrm{AT}, \mathrm{CB}(\mathrm{B}, \mathrm{F}), \mathrm{CB}(\mathrm{B}, \mathrm{F}, \mathrm{M})$ & $\mathrm{AT}, \mathrm{CB}(\mathrm{B}, \mathrm{F}), \mathrm{CB}(\mathrm{B}, \mathrm{F}, \mathrm{M}), \mathrm{CB}(\mathrm{F}), \mathrm{LIOP}$ \\
\hline $\mathrm{Sr}$ & 38 & & $\mathrm{CB}(\mathrm{F}), \mathrm{CB}(\mathrm{S}), \mathrm{CB}(\mathrm{PIC}), \mathrm{XB}(\mathrm{F})$ \\
\hline $\mathrm{Y}$ & 39 & & $\mathrm{CB}(\mathrm{B}, \mathrm{F})$ \\
\hline $\mathrm{Zr}$ & 40 & & $\mathrm{CB}(\mathrm{B}, \mathrm{F})$ \\
\hline $\mathrm{Nb}$ & 41 & & $\mathrm{CB}(\mathrm{B}, \mathrm{F})$ \\
\hline Mo & 42 & & $\mathrm{CB}(\mathrm{B}, \mathrm{F})$ \\
\hline $\mathrm{Ru}$ & 44 & $\mathrm{XB}(\mathrm{F})$ & $\mathrm{XB}(\mathrm{F})$ \\
\hline $\mathrm{Pd}$ & 46 & & $\mathrm{HC}$ \\
\hline $\mathrm{Ag}$ & 47 & RIS(IG) & $\mathrm{CB}(\mathrm{F}), \mathrm{RIS}(\mathrm{IG})$ \\
\hline $\mathrm{Cd}$ & 48 & $\mathrm{CB}(\mathrm{B}, \mathrm{F})$ & $\mathrm{CB}(\mathrm{B}, \mathrm{F}), \mathrm{HTC}$ \\
\hline In & 49 & & $\mathrm{CB}(\mathrm{F})$ \\
\hline Sn & 50 & & $\mathrm{CB}(\mathrm{F}), \mathrm{PLID}, \mathrm{XB}(\mathrm{F})$ \\
\hline $\mathrm{Te}$ & 52 & & HC, PLID, SG \\
\hline $\mathrm{Xe}$ & 54 & & $\mathrm{CB}(\mathrm{S})$ \\
\hline Cs & 55 & & $\mathrm{CB}(\mathrm{F}), \mathrm{LIOP}, \mathrm{OLC}$ \\
\hline $\mathrm{Ba}$ & 56 & & $\mathrm{CB}(\mathrm{B}, \mathrm{F}), \mathrm{CB}(\mathrm{F}), \mathrm{CB}(\mathrm{PIC}), \mathrm{XB}(\mathrm{F})$ \\
\hline $\mathrm{La}$ & 57 & & $\mathrm{CB}(\mathrm{F})$ \\
\hline $\mathrm{Ce}$ & 58 & & $\mathrm{CB}(\mathrm{B}, \mathrm{F}), \mathrm{CB}(\mathrm{F})$ \\
\hline $\mathrm{Nd}$ & 60 & & $\mathrm{CB}(\mathrm{F}), \mathrm{XB}(\mathrm{RIS}), \mathrm{RIS}$ \\
\hline $\mathrm{Pm}$ & 61 & & $\mathrm{CB}(\mathrm{F})$ \\
\hline $\mathrm{Sm}$ & 62 & & $\mathrm{CB}(\mathrm{F}), \mathrm{RIS}, \mathrm{XB}(\mathrm{F})$ \\
\hline $\mathrm{Eu}$ & 63 & & $\mathrm{CB}(\mathrm{F}), \mathrm{IT}(\mathrm{DR}), \mathrm{RIS}, \mathrm{RIS}(\mathrm{IS})$ \\
\hline $\mathrm{Gd}$ & 64 & & RIS, RIS(IS) XB(F) \\
\hline $\mathrm{Tb}$ & 65 & & RIS \\
\hline Dy & 66 & & $\mathrm{CB}(\mathrm{F}), \mathrm{XB}(\mathrm{F})$ \\
\hline Ho & 67 & & $\mathrm{CB}(\mathrm{F}), \mathrm{RIS}$ \\
\hline Er & 68 & & $\mathrm{CB}(\mathrm{F})$ \\
\hline $\operatorname{Tm}$ & 69 & & RIS, RIS(IS) \\
\hline $\mathrm{Yb}$ & 70 & & $\mathrm{CB}(\mathrm{B}, \mathrm{F}), \mathrm{CB}(\mathrm{F}), \mathrm{CB}(\mathrm{RIS}), \mathrm{GC}(\mathrm{F}), \mathrm{RIS}(\mathrm{IS}), \mathrm{XB}(\mathrm{F})$ \\
\hline $\mathrm{Lu}$ & 71 & & $\mathrm{CB}(\mathrm{F})$ \\
\hline $\mathrm{Hf}$ & 72 & & $\mathrm{CB}(\mathrm{B}, \mathrm{F}), \mathrm{CB}(\mathrm{F}), \mathrm{CB}(\mathrm{PIC}), \mathrm{XB}(\mathrm{F})$ \\
\hline $\mathrm{Ta}$ & 73 & & $\mathrm{CB}(\mathrm{B}, \mathrm{F}), \mathrm{XB}(\mathrm{F})$ \\
\hline $\mathrm{W}$ & 74 & $\mathrm{XB}(\mathrm{F})$ & $\mathrm{XB}(\mathrm{F})$ \\
\hline $\operatorname{Re}$ & 75 & & $\mathrm{HC}, \mathrm{XB}(\mathrm{F})$ \\
\hline Os & 76 & & $\mathrm{HC}$ \\
\hline Ir & 77 & & PLID \\
\hline $\mathrm{Pt}$ & 78 & 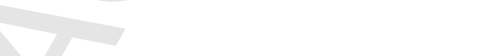 & PLID, RIS \\
\hline $\mathrm{Au}$ & 79 & & CB(F), HTC, HTC, PLID, RIS \\
\hline $\mathrm{Hg}$ & 80 & $\nabla$ & $\mathrm{CB}(\mathrm{F}), \mathrm{HTC}, \mathrm{RADOP}$ \\
\hline $\mathrm{Tl}$ & 81 & 2SF, RIS(IS) & 2SF, CB(F), RIS(IS) \\
\hline $\mathrm{Pb}$ & 82 & & $\mathrm{CB}(\mathrm{F}), \mathrm{RIS}(\mathrm{IS}), \mathrm{XB}(\mathrm{F})$ \\
\hline
\end{tabular}




\begin{tabular}{llll} 
Bi & 83 & & GC(F), RIS(IS) \\
Po & 84 & RIS(IS) & RIS(IS), XB(F) \\
Rn & 86 & & CB(F), CB(S), RADOP \\
Fr & 87 & AT, CB(B,F,M), CB(B,RIS $)$ & AT, CB(B,F,M), CB(B,RIS), CB(F), LIOP \\
Ra & 88 & & CB(F) \\
Th & 90 & IT(F), RIS(IG), XB(RIS) & IT(F), RIS(IG), XB(RIS) \\
U & 92 & & SG, XB(F) \\
Np & 93 & SG \\
Pu & 94 & SG \\
Am & 95 & ABMR, GC(OP), RIS (GC), SG \\
Cm & 96 & SG \\
Bk & 97 & SG \\
Cf & 98 & SG \\
Es & 99 & SG \\
Fm & 100 & RIS(GC) \\
\hline \hline
\end{tabular}

The collinear beam technique, and the variants thereof, has been applied to the majority of elements (and to the vast majority of those with stable isotopes). No relative decrease in the application of this technique can be identified in recent studies and the method remains popular for the spectroscopy of short-lived isotopes. Other techniques, notably crossed-beam, traps and variants of laser ion sources appear, when taken together, as approximately equal in their applicability by chemical element. Substantial overlap of the species studied however exists. Significant regions of the periodic table (and nuclear chart) have no reports of optical measurements for radioactive isotopes. The regions are the non-metals (excluding noble gases) and a number of the late $d$-shell refractory elements (those beyond the mid-shell). The prospects for the future study of these elements is returned to in Section 8.

In the preceeding sections minimum beam intensities extracted from the literature are presented. A number of the variants of collinear spectroscopy, Sections 3.4, 3.4.4 and 3.5.2, quote minimum fluxes of $\sim 100$ ions per second for studied systems with hyperfine structure and, either directly quote or can infer, $\sim 10$ ions per second for even-even isotopes. In-source RIS, Section 3.5.1, is quoted with an order of magnitude lower minimum intensity requirement. The atom and ion traps of Section 3.5.1 can be loaded at a few percent or greater efficiency once the sample has been either stopped or slowed to a few tens of keV energy (neglecting any metastable population efficiency concerns). The slowing from $\sim \mathrm{GeV}$ to $\sim \mathrm{keV}$ energies can however present a major efficiency loss especially for high energy species not stopped in solid material. Progress in addressing this issue is highlighted in Section 3.7.

The spectroscopies and efficiencies reported can routinely be seen to provide measurements of optical frequency splittings at $1-10 \mathrm{MHz}$ precision (for the Doppler-reduced techniques). The high precision techniques outlined in Sections 3.4.1 and 3.6 can improve these accuracies by $\sim 2$ orders of magnitude or further in the case of radio-frequency spectroscopy where $1-10 \mathrm{kHz}$ precision can be achieved for short-lived isotopes.

Required experimental sensitivity, precision and selectivity are critically determined by the spectroscopic challenge undertaken both in the nature and scale of the observation and in the, often production related, backgrounds encountered. Production facilities are considered in the next section. The production mechanisms used determine a critical further parameter for the study of short-lived nuclei the minimum species lifetime measurable. A majority of the facilities and spectroscopies now report, in perhaps occassionally favourable species, $\sim 1-10 \mathrm{~ms}$ as the minimum lifetime.

The lifetime limit permits the spectroscopy, and future spectroscopy, of vast numbers of isotopes and isomers but will in the longer term represent a critical limitation in the field. Perhaps reassuringly, the present typical limit can be noted to be far above that of the shortest species studied optically, the 1 microsecond ${ }^{85 m} \mathrm{Rb}$ isomer [73, 292]. A significant reassessment of the isomer shift between References 


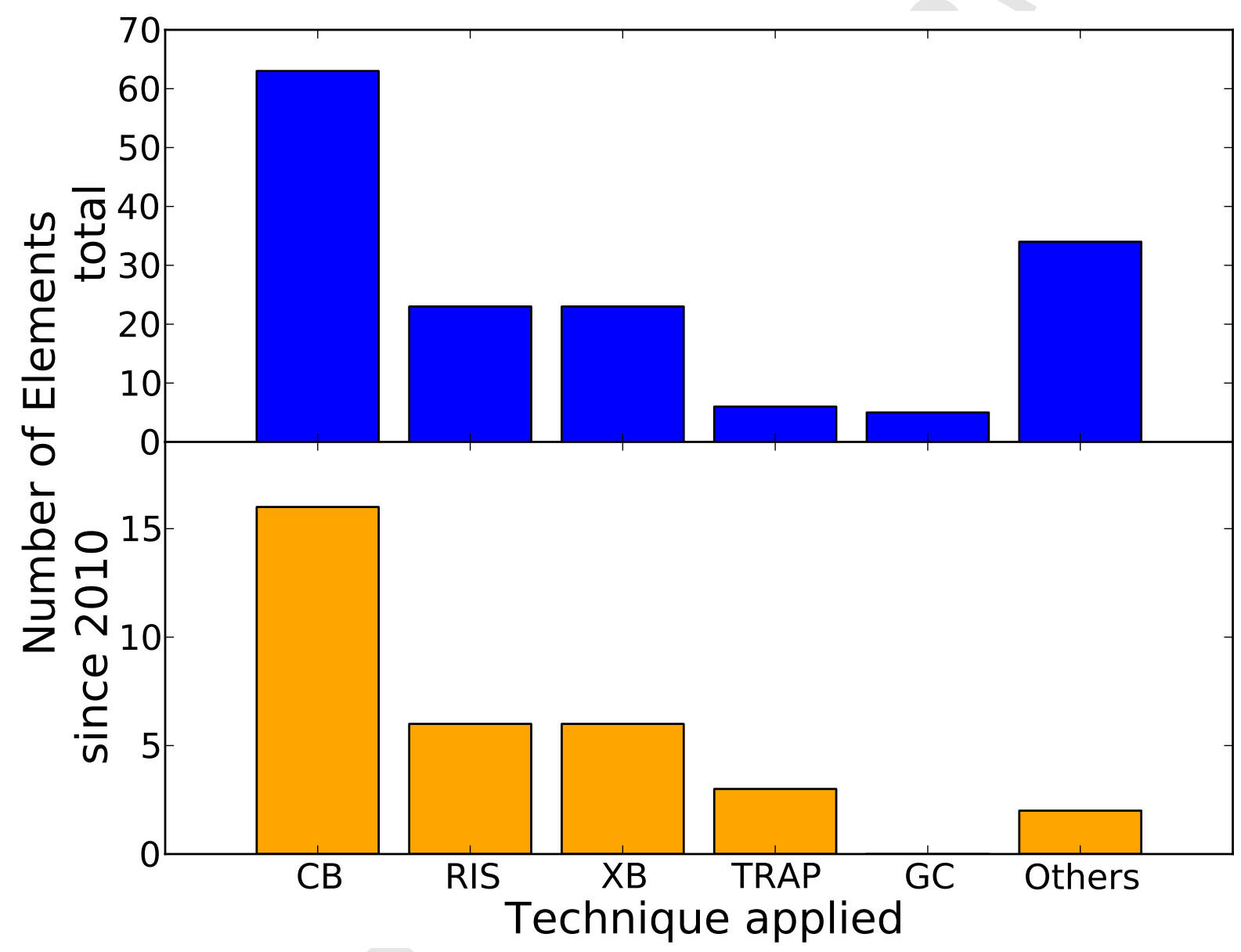

Figure 9: Deployment of major techniques to chemical elements (abbreviations following those of Table 2) for, top, all reports of radioactive isotope measurements and, bottom, those classed as recent in Table 1 . 
[292] and [73] does however council caution in accepting these works as entirely successful. 


\section{On-line laser spectroscopy facilities}

The 1979 review of the field by Jacquinot \& Klapisch [1] noted that, "a vast new field [has now] opened up" which a decade later, when reviewed by Otten [2], had matured to a large number of productive experiments, most notably at the ISOLDE facility, CERN, (Section 4.1.6). New facilities, upgrades to existing ones, and moves of experimental stations have been realised, planned and reviewed since [3-5]. The on-line isotope separator became the favoured, and almost exclusive, site for laser spectroscopy of radioactive nuclei. Possible future sites, which will include fragmentation separators, recoil separators and storage rings, are highlighted in this section and Section 8. The developments reflect advances in laser systems, ion sources, ion manipulation and primary production accelerator technology. Here we summarize the present and planned on-line laser facilities with emphasis on the advances and techniques in use at each facility.

\subsection{Present and planned facilities}

Table 3: Summary and status of the present and planned on-line laser facilities. The production mechanisms follow those described in Section 3.3 with $F F$ denoting fast fission and fragmentation. The electron-cyclotron resonance (ECR) ion source, forced electron beam inducedarc discharge (FEBIAD) ion source, laser ion source (LIS) and trap (LIST) are described in Section 3.3.

\begin{tabular}{lllll}
\hline \hline Facility & Status & Production & Ion Sources & References \\
\hline ALTO & Operational & Uranium photo-fission & Thermal & {$[293]$} \\
ANL, ATLAS & Operational & Light-ion & ECR ion source & {$[294]$} \\
ANL, CARIBU & Operational & Spontaneous fission & Gas cell & {$[216]$} \\
FAIR & Planned & In-flight fragmentation & Gas cell & {$[295]$} \\
GALS, DUBNA & Planned & Deep inelastic & Ion guide, LIS & {$[296]$} \\
GSI, ESR & Operational & In-flight fragmentation & Storage ring & {$[297]$} \\
GSI, HITRAP & Operational & In-flight fragmentation & Storage ring & {$[269]$} \\
GSI, SHIP & Operational & Light-ion, Heavy-ion & Gas cell & {$[298]$} \\
ISOLDE, HIE-ISOLDE & Operational & FF, Spallation & Thermal, FEBIAD & {$[299]$} \\
& & & LIS and LIST & \\
JYFL, IGISOL-4 & Operational & Light-ion, Heavy-ion, fisson & Ion guide & {$[300]$} \\
MSU, BECOLA & Operational & In-flight fragmentation & Gas cell & {$[301]$} \\
MSU, FRIB & Planned & In-flight fragmentation & Gas cell & {$[302]$} \\
PNPI & Operational & FF, Spallation & Thermal, LIS & {$[303]$} \\
RIKEN, RIBF & Operational & In-flight fragmentation & Gas cell & {$[304]$} \\
RIKEN, KISS & Commissioning & Deep inelastic & Ion guide, LIS & {$[305]$} \\
RISP & Planned & FF, Spallation & Thermal, FEBIAD & {$[306]$} \\
& Planned & d,n-induced fission & and LIS & Thermal \\
SPIRAL 2, GANIL & fragmentation & Ion guide, LIS & {$[307]$} \\
TRIGA-SPEC & Commissioning & n-induced fission & Aerosol-gas jet & {$[223]$} \\
TRIUMF & Operational & FF, Spallation & Thermal, FEBIAD & {$[308]$} \\
& & & LIS and LIST & \\
\hline \hline
\end{tabular}




\subsubsection{ALTO}

The ALTO facility [293] at Orsay (France) is commissioned and has delivered first beam. The separator exploits a photo-fission ion source in which an intense nuclear fission rate ( $10^{11}$ fissions per second) is achieved by the multiple secondary interactions of $10 \mu \mathrm{A}$ of $50 \mathrm{MeV}$ electrons. A laser ion source has demonstrated the efficient, selective ionisation of neutron-rich Ga isotopes [309] and further production schemes are under development for the SPIRAL2 facility at GANIL (Section 4.1.12).

\subsubsection{ANL}

ATLAS, the Argonne Tandem Linear Accelerator Stage [294], is a superconducting linear accelerator for heavy ions at Coulomb barrier energies. It has been used to produce the two-neutron halo nucleus ${ }^{6} \mathrm{He}$ for laser spectroscopy in a magneto-optical trap (MOT) [13].

The Californium Rare Ion Breeder Upgrade (CARIBU) project [216] at the Argonne National Laboratory aims to provide beams of extremely neutron-rich isotopes from the captured and cooled fragments produced in the spontaneous fission of ${ }^{252} \mathrm{Cf}$. $\mathrm{A} \sim 1$ Curie source is housed in a gas catcher and separated species are available for re-acceleration to high-energy or for delivery to a low-energy experimental hall and proposed laser spectroscopy station.

\subsubsection{FAIR}

Intense interest focuses on the future FAIR facility to be built at the GSI site. A Technical Design Report by the MATS (nuclear mass measurements) and LASPEC (laser spectroscopy) collaborations was completed in 2009 and published in 2010 [295]. Both proposed projects, part of the NUSTAR (NUclear STructure, Astrophysics and Reactions) collaboration, intend to take radioactive ion beam from a gas stopper positioned at the end of the super fragment separator [310]. Recently, a prototype cryogenic stopping cell (CSC) for FAIR has been successfully tested at the current fragment recoil separator, GSI [311]. The intense output of the future separator will be stopped, cooled and bunched (and, with high likelihood, mass separated) prior to delivery to the low energy stations. At the LASPEC station two beamlines are proposed to enable fast atomic and fast ion laser spectroscopy. Developments in collinear laser spectroscopy relevant for LASPEC have been recently reported [312].

\subsubsection{GALS, DUBNA}

A planned facility, the GAs cell based Laser ionisation Setup (GALS)[296] at the Joint Institute for Nuclear Research (JINR), Dubna, proposes to exploit available beams from the U-400M cyclotron in deep-inelastic, multi-nucleon transfer reactions to study exotic neutron-rich nuclei ("north-east" of ${ }^{208} \mathrm{~Pb}$ ). Products from $4.5-9 \mathrm{MeV} /$ nucleon heavy-ion collisions, such as ${ }^{136} \mathrm{Xe}$ on ${ }^{208} \mathrm{~Pb}$, are to be captured in a gas cell and selectively laser-ionised in a SPIG extraction system (Section 3.7.2). A high repetition $(10 \mathrm{kHz}) \mathrm{Nd}$ :YAG pumped dye laser system has been selected to provide the resonant ionisation schemes. The proposed research programme includes the future use of actinide targets.

\subsubsection{GSI}

At GSI, the accelerator complex consisting of the UNILAC and SIS is used to produce highly-charged ions (HCI) up to $\mathrm{U}^{92+}$ by passing a $400 \mathrm{MeV} / \mathrm{u}$ beam through a stripper foil. HCI presently offer a unique opportunity for fundamental tests of quantum electrodynamics under conditions of extreme fields that exist around their nuclei. Future implications for nuclear structure are considered in Section 8.

Two experimental approaches have been realized at GSI that utilise laser spectroscopy in the study of HCI. In the first, LIBELLE, laser spectroscopy is performed in the experimental storage ring, ESR, 
on the relativistic $(400 \mathrm{MeV} / \mathrm{u})$ ions with high power lasers. Traditionally, measurements have been carried out on H-like and Li-like bismuth with the transition in the latter found in 2011 [297, 313, 314]. The successor to the LIBELLE experiment, SpecTrap (Spectroscopy Trap), is expected to provide a relative accuracy two to three orders of magnitude greater. SpecTrap is an experiment associated with the HITRAP (highly charged ion trap) facility, an experimental platform constructed for a number of experiments on HCI almost at rest, providing access to precision experiments in atomic, nuclear and solid state physics [269]. HITRAP and ESR experiments will in future be undertaken as part of the SPARC (Stored Particles Atomic Physics Research Collaboration), FAIR.

Further efforts at GSI target the first spectroscopic investigations on the heaviest elements, namely No $(Z=102)$ and $\operatorname{Lr}(Z=103)$, discussed separately in Section 7.1.3. Investigation of (thus far unobserved) atomic levels associated with the transfermium elements are performed in a buffer gas cell located behind the velocity filter SHIP. The current status has been summarized by Laatiaoui [298].

\subsubsection{Isolde, HIE-Isolde, CERN}

In 2017 the ISOLDE facility at CERN will celebrate 50 years of operation, an achievement that will include some 40 years of on-line laser spectroscopic studies. Essentially all, bar a specialised few, of the techniques presented here are in use, or have been in use, at the facility. The developments and exploitations include on-line atomic beam magnetic resonance, collinear laser spectroscopy, collinear resonance ionisation spectroscopy CRIS, pulsed laser-induced desorption PLID, laser ion sources and laser polarisation NMR studies. The suite of experimental stations benefit from two ISOL targets and separators (one general purpose and one high resolution). Either target station can receive beam from the CERN proton-synchrotron booster capable of delivering $1.4 \mathrm{GeV}$ protons in bunches of $\sim 2 \times 10^{13}$ protons per bunch.

In 2014 a shutdown and upgrade period ("Long Shutdown 1") has finished and the HiE-ISOLDE project has commenced [299]. The upgrades to the facility for this project enhance the low-energy beam production (as well as providing the increase in performance desired for efficient and intense delivery of re-accelerated, high-energy, beams). Prior to the shutdown the RILIS laser ion source was used in delivery of over 3000 hours of radioactive beam per year (facilitating a great range of nuclear spectroscopies beyond the scope of this review) [315]. The reduction in isobaric background, achieved by the laser selectivity, critically reduces non-resonant photon backgrounds in collinear spectroscopy and, of particular pertinence to present developments at the facility, prevents space charge limits from hindering the loading of on-line ion traps and cooler-bunchers.

The RFQ cooler-buncher, ISCOOL [259], has been in operation at IsOLDE since 2008 and has permitted both bunched beam spectroscopy and, recently, the first results from the collinear resonance ionisation spectroscopy of bunched radioactive beams, Section 3.5.2.

\subsubsection{JYFL}

The new IGISOL-4 (Ion Guide Isotope Separator On-Line) facility [300] at the University of Jyväskylä Accelerator Laboratory, JYFL (Finland), reported their first, post commissioning, laser spectroscopy results in 2013. The commissioned facility is equipped with a dedicated MCC-30 light-ion cyclotron, for driving proton-induced fission, while still capable of receiving beams from an existing K130 cyclotron. A suite of $10 \mathrm{kHz} \mathrm{Nd}$ :YAG pumped solid state lasers are available for laser ionization, resonance ionization spectroscopy and methods of optical pumping. An extensive ultra-low-energy ion beam delivery section is designed to enable optical spectroscopy on bunched ensembles at energies from $800 \mathrm{eV}$ to $30 \mathrm{keV}$.

The ion-guide ion source, developed at the facility [215, 316], and RFQ cooler-buncher [127] have been exploited particularly for the study of elements in the two refractory regions, $\mathrm{Y}-\mathrm{Rh}$ and $\mathrm{Hf}-\mathrm{Pt}$. 


\subsubsection{MSU}

A gas stopper coupled to a fragment separator, similar to that proposed for use at FAIR, has been realised and developed at the LEBIT (low-energy beam and ion trap) facility, NSCL, Michigan State University. Extraction efficiencies close to $10 \%$ have been achieved for weak exotic beams and have been studied in a dedicated Penning trap mass spectrometer [317]. A new collinear laser beamline, BECOLA (beam cooling and laser spectroscopy) [301], is served from a high intensity, cryogenic cooler-buncher. Off-line commissioning, concentrating on elements ${ }_{25} \mathrm{Mn}$ and ${ }_{26} \mathrm{Fe}$ has been completed [52] and very recently complemented with the first study of the radioactive isotopes ${ }^{36,37} \mathrm{~K}$, produced via projectile fragmentation [41, 42]. MSU will host the future FRIB facility [302] - a linac driven, high-energy facility with an extensive low-energy area under construction to be served by linear gas stoppers and an inverse cyclotron-stopper.

\subsubsection{PNPI}

At the Investigation of Radioactive Isotopes on Synchrocyclotron (IRIS) facility, Petersburg Nuclear Physics Institute, Gatchina (Russia) [318], beam from a $1 \mathrm{GeV}$ proton synchrocyclotron impinging on a uranium carbide, $\mathrm{UC}_{\mathrm{x}}$, target forms the production platform for an isotope separator, IRIS (at which the first use of resonance laser ionisation for nuclear spectroscopy was demonstrated in 1983 [235]). The adaption of the technique to in-source laser ionisation vastly extended the measurements possible and demonstrated [110], that when used in conjunction with decay detection, provides a spectroscopy with a sensitivity that is still unparalleled.

\subsubsection{RIKEN}

The RIBF (radioactive ion beam facility), RIKEN, currently produces the highest intensity of highly exotic nuclei anywhere in the world through in-flight fission and fragmentation. Two "slow" beam experiments are coupled to the BigRIPS fragment separator [304]. A non-optical electron scattering ion trap SCRIT (Self-confining radioactive ion target) [319] is also under development at this facility and is discussed in Section 8. Both slow beam experiments, the SLOWRI (slow radioactive nuclear ion beam) gas cell and PALIS (parasitic radioactive beam by laser ionisation source) platform, couple to optical stations or provide direct spectroscopic opportunities. The SLOWRI gas cell prototype [217] demonstrated the first use of an rf-carpet guide and provided radioactive Be isotopes for an ion trap rf-double pumping experiment [23]. The PALIS development [218] aims to increase the stopping power of the gas cell such that a compact device can be installed beyond the separator magnet of BigRIPS and, through a side entrance window, capture "dumped" beam parasitically during the operation of the separator.

In a unique spectroscopy, the Optical Radioisotope-atom Observation in Condensed Helium as Ioncatcher (OROCHI) experiment at RIKEN [320] stops the RIPS beam in superfluid He at $1.8 \mathrm{~K}$. In the superfluid He the absorption and emission profiles of atomic transitions broaden and separate enabling low background observation of laser-induced fluorescence. To make hyperfine structure measurements a laser-radio-frequency double-resonance spectroscopic approach is adopted. The spectroscopy (including the essential evaluation of the He pressure shifts) has been proven in a number of stable elements (Rb, $\mathrm{Cs}, \mathrm{Ag}$ and $\mathrm{Au}$ ) and has been used on-line in the nuclear spin measurements of ${ }^{84-87} \mathrm{Rb}$ [320].

A separate development, the KEK Isotope Separation System (KISS), is presently being commissioned at RIKEN [305]. The laser ion-guide based separator aims to provide target-like, multi-nucleon transfer products (in a manner similar to that proposed in Section 4.1.4). Accelerated heavy-ion beams will be taken from the RIKEN ring cyclotron and used to produce neutron-rich species for intra-cell ionisation. The selected laser system consists of 2 excimer pumped dye lasers and both off-line and 
on-line testing with stable ${ }^{56} \mathrm{Fe}$ has been completed. The KISS facility opened for user proposals in March 2015.

\subsubsection{RISP}

The Rare Isotope Science Project (RISP), South Korea proposes the construction of a large scale facility that includes a $600 \mathrm{MeV}$ proton driven ISOL facility with a multi-purpose laser station. The proposed ISOL includes a high resolution mass separator (with RFQ pre-cooler) that, on one spectroscopy line, will feed an RFQ cooler-buncher and multi-reflection TOF spectrometer. This line further feeds twin laser and mass spectroscopy stations. The former is proposed to be multi-purpose laser line with all variants of collinear beam spectroscopy open to the user. A full Baseline Design Study may be found in Reference [306].

\subsubsection{SPIRAL 2, GANIL}

The GANIL facility hosted the apparatus used in the charge radius determination of ${ }^{6} \mathrm{He}$ at Argonne National Laboratory [13], for the subsequent measurement of ${ }^{8} \mathrm{He}$ [14]. In the latter experiment, both ${ }^{6} \mathrm{He}$ and ${ }^{8} \mathrm{He}$ were simultaneously produced from a primary beam of $75 \mathrm{MeV} / \mathrm{u}{ }^{13} \mathrm{C}$ impinging on a heated graphite target.

In 2005 GANIL announced the decision to construct the SPIRAL2 facility. The new facility will see a high intensity linac drive two ion sources and deliver high intensity stable ion beams to the $\mathrm{S}^{3}$ (super separator spectrometer) installation. Short-lived radioactive ion beams from $\mathrm{S}^{3}$, a uranium-carbide ion source and the existing SPIRAL facility can be delivered to the DESIR (Decay, Excitation and Storage of Radioactive Ions) low energy area [307]. This area is envisaged to include two laser spectroscopy lines providing both variant collinear spectroscopy and polarised beam $\beta$-NMR studies. In addition to laser spectroscopy at DESIR, laser resonance ionization will take place at $\mathrm{S}^{3}$. Reaction products separated in-flight will be stopped and neutralized in a specially designed gas cell placed at the focal plane [280]. The species of interest will be selectively re-ionized, either in the gas cell or in the expanding gas jet. The source will be used both for spectroscopy and, at a later stage, to provide pure beams for operations at DESIR.

\subsubsection{TRIGA-SPEC}

A combined mass and laser spectrometer TRIGA-SPEC [223] has been commissioned at the Mainz TRIGA reactor. The stations can receive beam from neutron-induced fission of actinide targets and from samples of reactor-bred transactinide elements. The multi-purpose spectrometer serves as a prototype for the MATS and LASPEC projects highlighted in Section 4.1.3.

The layout of the facility, with a central RFQ cooler-buncher serving laser and mass spectrometry stations, is representative of the majority of the recently commissioned or proposed facilities and is presented in Figure 2.

\subsubsection{TRIUMF}

Recent effort at the ISAC facility TRIUMF [308] has concentrated on the development and use of a laser ion source, a collinear laser beamline (previously exploited in the preparation of laser-polarised lithium) and a Fr atom trapping facility. In the first, a high-efficiency laser ion source has proved successful in providing both spectroscopic data and on-line production for ${ }_{85} \mathrm{At}[321,322]$ and ${ }_{89} \mathrm{Ac}$ as well as medium mass nuclei including ${ }_{32} \mathrm{Ge}$ [253]. In the second, collinear laser spectroscopy, using bunch ion ensembles and a chopped laser beam [157], have provided a high-sensitivity spectroscopy that has been used in the study of $N=Z^{74} \mathrm{Rb}[71],{ }^{98} \mathrm{Rb}[72]$ and ${ }_{87} \mathrm{Fr}[157,162]$ isotopes at extreme neutron-deficiency. Finally, 
the Francium Trapping Facility [265] of the FrPNC collaboration is commissioned and described in detail in Section 3.6.

The "2010 - 2015" plan at the facility proposes the commissioning of an electron LINAC and development of a photo-fission ion source (similar to that developed at the ALTO facility 4.1.1).

\subsection{Recent changes}

The status of the LISOL facility at Louvain-la-Neuve and the Oak Ridge National Laboratory (ORNL) have, in the reviewed period, changed.

Apparatus from the Leuven isotope separator on-line (LISOL) facility (CRC, Louvain-La-Neuve, Belgium) are to move and form the basis of HELIOS at the $\mathrm{S}^{3}$ separator at GANIL [280], Section 4.1.12. During two decades of development prior to the move the LISOL facility demonstrated, for the first time, both efficient laser ionisation in an ion-guide source and, more recently, highly sensitive optical spectroscopy for the study of short-lived exotic nuclei. The earlier development of the SPIG increased both the efficiency and mass resolving power of the separator [323] and is itself now exploited in spectroscopic developments.

At the Oak Ridge National Laboratory (ORNL) a laser ion source, at the Holifield Radiaoctive Ion Beam Facility (HRIBF), has been developed and results have been recently reported [324]. HRIBF ceased operations April 2012. 


\section{Light Mass nuclei}

Nuclei in the region $Z<28$ that have been studied via laser spectroscopic techniques may be grouped into several distinct areas of physics. The lightest nuclei with $2 \leq Z \leq 4$ are dominated by the one- and two-neutron halo structures. Above this, nuclei around $Z \approx 12$ and $N \approx 20$ fall in a region of the chart known as the island of inversion where much experimental effort has been applied in order to probe the onset and evolution of shell structure of the neutron rich nuclei as well as to map the boundary of the island. Mean-square charge radii of nuclei in the Ca region have been subject to extensive experimental and theoretical investigations, with existing data covering the full $\nu f_{7 / 2}$ shell which has recently been complemented by an extensive chain of $\mathrm{K}$ isotopes crossing the $N=28$ shell closure. The systematic changes in the root mean-square charge radii as well as extraction of nuclear moments are summarized in this section.

\section{$5.1 \quad$ Results and recent progress}

\subsubsection{The proton radius puzzle}

"How big is the proton?", this was the fundamental question posed in the title of the news feature in 2013 which accompanied the latest precision measurement of the charge radius of the proton via observation of the $2 S-2 P$ transition frequencies of muonic hydrogen [325]. The result had an uncertainty $40 \%$ lower than previous work (by the same collaboration) [326] and was at a variance with the recommended value from CODATA 2010 [327] by 7 standard deviations.

Muonic hydrogen consists of a negative muon $\mu^{-}$orbiting a proton. Due to a mass difference $m_{\mu} / m_{e} \sim 207$, the Bohr radius of the muon is some 200 times smaller than that of the electron and therefore considerably more sensitive to the effects related to the finite size of the proton. Consequently, the effects on the muonic $S$ states are enhanced, indeed for the $2 S$ state the finite-size effect is about $2 \%$ of the Lamb shift (the energy difference between the $2 S$ and $2 P$ states).

At the Paul Scherrer Institute (PSI) in Switzerland, a novel beamline was built especially for transporting low-energy negative muons which are stopped in $1 \mathrm{mbar}_{2}$ gas. Approximately $1 \%$ of the highly excited muonic hydrogen atoms cascade down into the metastable $2 S$ state (the rest to the ground state). Pulsed laser light tunable from $5.5 \mu \mathrm{m}$ to $6.1 \mu \mathrm{m}$ (created by Raman mixing of a pulsed amplified Ti:sapphire laser) was used to induce excitations from both hyperfine states of the $2 S$ level to the $2 P$ level, with excitation signaled by the detection of a $1.9-\mathrm{keV} \mathrm{K}_{\alpha} \mathrm{X}$-ray which is emitted in the radiative $2 P-1 S$ deexcitation. This seminal experiment resulted in a precise measurement of the Lamb shift which, when combined with theory, led to a value for the proton charge radius of $r_{p}=0.84087(39)$ $\mathrm{fm}$ [325]. The CODATA 2010 value, an order of magnitude less precise with $r_{p}=0.8775(51) \mathrm{fm}$, is composed from precision Lamb shift measurements of several transitions in atomic hydrogen combined with elastic electron-proton scattering. These electron-based results are mutually compatible, with multiple independent electron-proton scattering experiments in agreement with each other as well as with several mutually consistent measurements of different transition frequencies in hydrogen. It is the striking discrepancy with the (more accurate) muonic results which is now refered to as the proton radius puzzle.

Recently, two review papers have comprehensively discussed the origins of the puzzle, summarizing the experimental and theoretical challenges [328, 329]. The "puzzle" in the situation arises as it seems highly unlikely that the experimental results are incorrect, and similarly, to assert that there is a problem with the quantum electrodynamical (QED) corrections which contribute to the Lamb shift (both electronic and muonic) also seems implausible (with a substantial volume of work having been done by more than one group, using different methods to evaluate the corrections). Speculations that the puzzle might originate from a difference between the electron-proton and muon-proton interactions violates the principle of lepton universality. This is an exciting possibility as it would indicate physics beyond the Standard Model. Indeed, any exotic explanations impact other purely muonic processes, 
for example the theory involved in the muon $g-2$ measurements [330]. Tensions exist between the different data sets used to extract the proton charge radius from elastic electron scattering and how one should combine and evaluate the systematic uncertainties has been the subject of much recent discussion [331, 332]. Based on these considerations a new recommendation for a global average value of $r_{p}=0.8790(110) \mathrm{fm}$ has been suggested [332]. This more conservative approach to the treatment of scattering data still results in a $3.5 \sigma$ discrepancy between electron-proton scattering and muonic hydrogen measurements, which increases to $5.7 \sigma$ when combining the scattering and atomic Lamb shift measurements.

The proton radius puzzle persists, with important implications in fields of high energy, nuclear and atomic physics. As discussed in [328, 329], new results are anticipated from hydrogen spectroscopy, including a new microwave measurement of the $2 S-2 P$ Lamb shift. Modelling of the Lamb shift is limited not only by the proton radius but also the Rydberg constant, $R_{\infty}$ [328]. Due to the correlation between these two parameters, new planned measurements to determine $R_{\infty}$ will help to shed light on the discrepancy. Muon-proton scattering should provide further insights, with an experiment planned at PSI to explore whether muonic hydrogen scattering provides the same answer as the existing muonic hydrogen spectroscopy.

Further spectroscopy, with exotic atoms, should significantly contribute to the solving of the puzzle. The CREMA (Charge Radius Experiment with Muonic Atoms) collaboration simultaneously measured $2 S-2 P$ transition frequencies for muonic deuterium, during the first muonic hydrogen run. These results are presently being analysed. The effect of the finite nuclear size increases from $2 \%$ in muonic hydrogen to $20 \%$ in muonic helium. A comparison of measured Lamb shifts in muonic atoms with the theoretical prediction gives very precise values of nuclear charge radii (proton, deuteron, ${ }^{3} \mathrm{He},{ }^{4} \mathrm{He}, \ldots$ ). The aim of CREMA is to measure the Lamb shift in $\mu^{3,4} \mathrm{He}^{+}$with relative accuracies of at least $50 \mathrm{ppm}$ [333], paving the way for deductions of the corresponding nuclear radii to a relative accuracy of at least $3 \times 10^{-4}$. The combination of such a measurement with those in electronic $\mathrm{He}^{+}$transitions should provide a path, via the $Z$ and $A$ dependencies, to resolving whether the theoretical or experimental investigations (or both) are deficient.

Lastly, as discussed in the next section, high precision laser spectroscopy has been performed on the neutron rich isotopes ${ }^{6,8} \mathrm{He}$ [14]. In order to deduce absolute radii it is necessary to know the absolute radius of the reference isotope ${ }^{4} \mathrm{He}$. A measurement of $\mu \mathrm{He}^{+}$will therefore contribute to nuclear structure physics. One may also forsee future transitions in muonic lithium, beryllium and boron ions, for which accurate measurements of electronic isotope shifts have been performed or are planned.

\subsubsection{Halo Nuclei (He, $\mathrm{Li}, \mathrm{Be})$}

The defining characteristic of a halo nucleus is that of a large spatial extension of valence nucleons in comparison to the nuclear core (potentially tunneling out from a nuclear core). Such a feature is characterised by a number of observables including a weak binding energy of the last nucleon(s), a large interaction cross section and a narrow momentum distribution of the nucleons following a breakup reaction. Thus far, halos have been observed in the form of either one- or two-nucleon systems confined to the lightest region of the nuclear chart, with neutron halos having been experimentally identified and predicted to exist in most nuclei near the neutron drip line [334]. Following the discovery of an exceptionally large interaction cross section for the ${ }^{11} \mathrm{Li}$ nucleus by Tanihata in 1985 [335], there has been a tremendous effort to produce and study halo nuclei with a number of spectroscopic probes as well as theoretical models. Throughout this period the field has been regularly reviewed, most recently by Tanihata et al in 2013 [334].

Soon after the discovery of the halo phenomenon, in-beam optical polarization combined with $\beta$ NMR spectroscopy was used to determine the nuclear moments of neutron-rich Li [336, 337] (and 


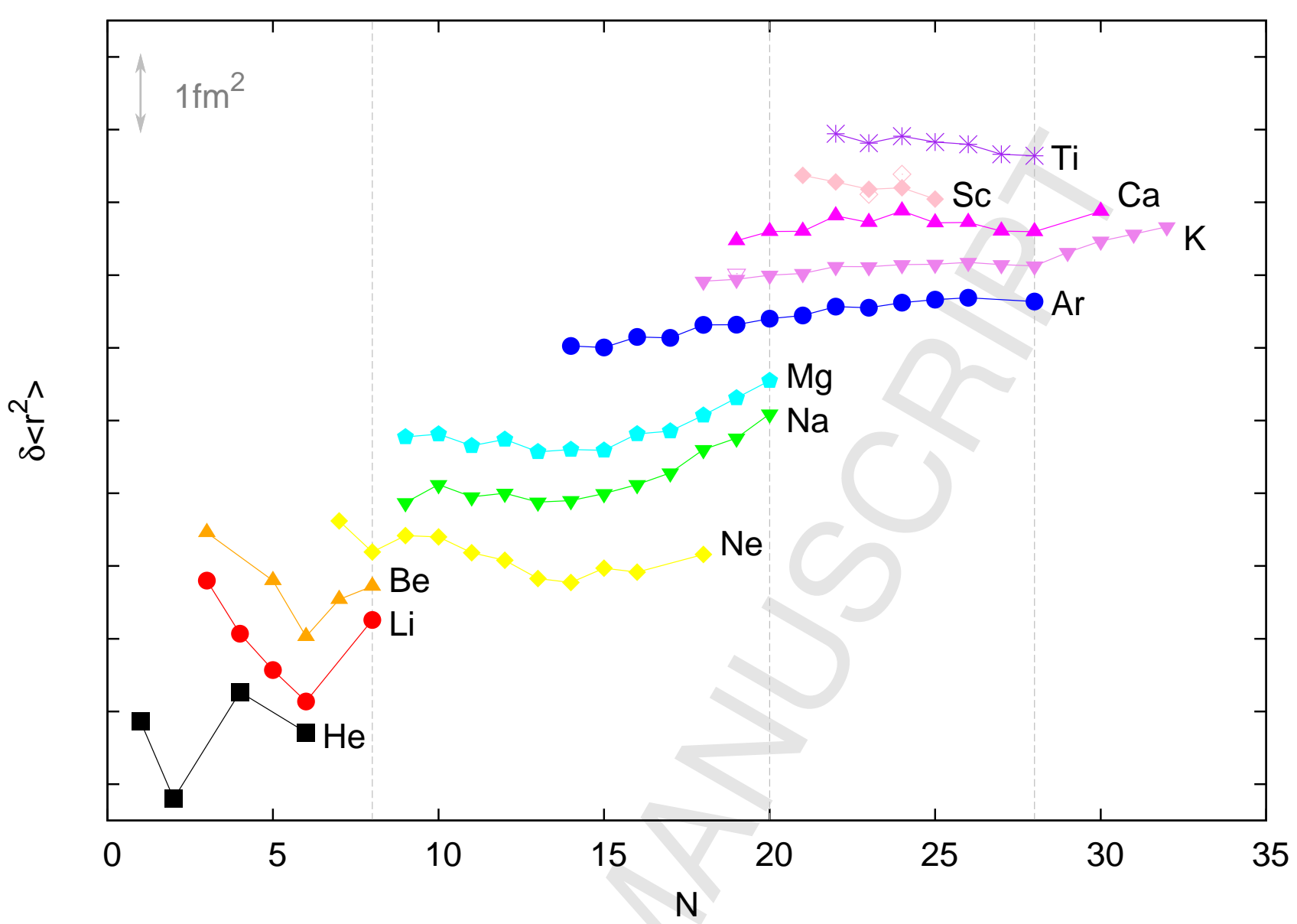

Figure 10: Changes in mean-square charge radii for the light mass nuclei from $\operatorname{He}(Z=2)$ to $\operatorname{Ti}(Z=22)$. The dotted lines represent the known shell closures at $N=8,20$ and 28. Elemental chains have been arbitrarily offset from each other for clarity. Colour on-line.

later Be [338]) rejecting the idea of a strongly deformed core being the explanation for the enhanced matter radii seen in ${ }^{11} \mathrm{Li}$. At that time however, neither theory nor experiment could provide the accuracy required to extract the charge radii which could be used to provide critical constraints on core modifications, testing correlations in the core + halo nucleon(s) system (i.e. the movement of the core around the centre of mass), as well as a possible contribution of core excitation.

The isotope shift of the lightest nuclei is dominated by the mass-shift component, this being of the order of $10 \mathrm{GHz} \cdot a \mathrm{mu}$ whereas the field shift is often significantly less than $1 \mathrm{MHz}$. The requirement for measurements with a precision of $10^{-5}$ or better is beyond the capability of many of the more standard experimental techniques used in the study of heavier nuclei (discussed in Section 3). Dedicated approaches for the lightest elements were therefore developed, pertinent details of which are discussed briefly. When combined with state-of-the-art Penning trap mass spectrometry and high precision atomic structure calculations, a detailed picture of the region has emerged [9].

A summary of the atomic structure theory including both QED and relativistic corrections, nuclear structure models as well as laser spectroscopic techniques may be found in [339]. Together with nuclear masses, charge radii and electromagnetic moments of the lightest elements they provide crucial benchmarks to test these theories. Excellent theoretical nuclear structure progress has also been made to understand the lightest elements, with approaches based on ab initio methods [340-342], the no-core 
shell model [343], fermionic molecular dynamics model [344, 345] and cluster models [346].

As discussed in Section 3.6, spectroscopy of the halo isotopes ${ }^{6,8} \mathrm{He}$ was performed within a magnetooptical trap [13, 14]. The results of the isotope shift measurements were later reevaluated following precision mass measurements at TITAN [347]. As seen in Figure 10 the charge radius increases from ${ }^{4} \mathrm{He}$ to ${ }^{6} \mathrm{He}$ then decreases as two more neutrons are added, at variance with the matter radii which slightly increase from ${ }^{6} \mathrm{He}$ to ${ }^{8} \mathrm{He}$ [339]. This is understood in terms of the correlations between the halo neutrons. In ${ }^{6} \mathrm{He}$ the recoil motion of the core against the two correlated neutrons smears out the charge distribution, whereas in ${ }^{8} \mathrm{He}$ the neutrons are distributed in more spherical symmetric manner, weakening the smearing of the charge in the core and thus resulting in a reduction of the charge radius. These effects are well reproduced with ab initio calculations [14].

Extensions of the atomic theory allowed for accurate mass shift calculations of three-electron systems such that valuable spectroscopy could be performed on the Li atom rather than the two-electron ionic system [348]. The production rates of ${ }^{11} \mathrm{Li}$ are however of order $10,000 / \mathrm{s}$ even at the most intense radioactive beam facilities in the world, and the spectroscopy requires a different approach to that of He. A technique involving Doppler-free two-photon excitation followed by resonance ionization and mass separation [15], was developed at GSI for the spectroscopy of ${ }^{6,7,8,9} \mathrm{Li}$ [17] and later transferred to TRIUmF for the measurement of ${ }^{11} \mathrm{Li}[19]$. In that work, the accelerated ion beam was implanted into a carbon foil and heated via a $\mathrm{CO}_{2}$ laser to $\sim 2000{ }^{\circ} \mathrm{C}$. The atoms quickly diffuse to the surface of the foil from which they are released into an ionization region in front of a quadrupole mass filter (QMF). Here the atoms were resonantly ionized in a three-step, four-photon scheme. In the first step, the Li atoms were excited in a standing wave laser field tuned to the $1 \mathrm{~s}^{2} 2 \mathrm{~s} \rightarrow 1 \mathrm{~s}^{2} 3 \mathrm{~s}$ atomic two-photon transition at $735 \mathrm{~nm}$. When this radiation was tuned in frequency to exactly half of the transition energy the nonrelativistic, first order Doppler shifts of the counter-propagating travelling waves that form the standing wave exactly cancel in the rest frame of the atom. This ensured that all atoms within the laser field were simultaneously available for the measurement. Once excited to the $1 \mathrm{~s}^{2} 3 \mathrm{~s}$ state the atom naturally relaxes through the $1 \mathrm{~s}^{2} 2 \mathrm{p}_{j}$ electronic level from where it is resonantly ionised via the $1 \mathrm{~s}^{2} 3 \mathrm{~d}$ state. The ions are mass-analysed using the QMF and counted. Isotope shift measurements are performed on the $2 \mathrm{~s} \rightarrow 3 \mathrm{~s}$ transition by scanning the 735 -nm laser light across the two-photon resonances. This technique resulted in an accuracy of $\sim 100 \mathrm{kHz}$ in the isotope shift for ${ }^{11} \mathrm{Li}$.

From these two experiments, the charge radii have been extracted and are seen to continually decrease in size from ${ }^{6} \mathrm{Li}$ to ${ }^{9} \mathrm{Li}$ (as shown in Figure 10), attributed to the strong clusterization [17]. Sanchez observed a significant increase in the charge radius of ${ }^{11} \mathrm{Li}$ [19], albeit far below that of the matter radius. In a comparison with a variety of models, the trend in the charge radii was most closely reproduced with a microscopic cluster model including core excitations (core polarization) by the halo neutrons, indicating that neutron correlations alone are not sufficient to explain ${ }^{11} \mathrm{Li}$. Later, the mass of ${ }^{11} \mathrm{Li}$ was precisely measured at TITAN which practically eliminated the contribution of the uncertainty arising from the mass in the charge radii determination [349].

Measurements at ISOLDE CERN using $\beta$-asymmetry detection of NMR on optically polarized beams provided precision measurements of the nuclear moments of ${ }^{8,9,11} \mathrm{Li}$ with an accuracy in the determination of all magnetic moments reaching the level of $10^{-5}$ and the quadrupole moments to the $1 \%$ level $[350,351]$. The magnetic moment of ${ }^{11} \mathrm{Li}$ is very close to the Schmidt value of a proton in the $\mathrm{p}_{3 / 2}$ shell, indicating a spherical nucleus, while the quadrupole moment of ${ }^{11} \mathrm{Li}$ is $\sim 10 \%$ larger than that of ${ }^{9} \mathrm{Li}$. Rather surprisingly, the cluster model which reproduces the charge radii fails to reproduce the quadrupole moments however the authors of ref. [351] present a phenomenological argument which ascribes the increase of the charge radius to a recoil effect caused by a spherical halo, with a resulting expansion of the (nonspherical) charge distribution. A detailed summary of the charge radii and ground state structure of lithium isotopes may be found in Nörtershauser et al [16].

It is only recently that the theoretical mass shift calculations have been performed with sufficient accuracy on four electron systems [352] and spectroscopy on Be thus far has been carried out on the 
three-electron $\mathrm{Be}^{+}$system. $\mathrm{Be}^{+}$ions offer an alkali-like structure with the $S_{1 / 2} \rightarrow P_{1 / 2}$ (D1) transition at $313 \mathrm{~nm}$, comfortably within the range of frequency doubled tunable lasers. In Section 3.4.1 a precision study of radioactive isotopes of $\mathrm{Be}^{+}$isotopes was highlighted. Experimentally isotopes in the study were produced at ISOLDE with a 1.4-GeV proton beam impinging on a uranium carbide target. Beryllium atoms, having diffused out of the target matrix were resonantly ionized using the laser ion source RILIS and following acceleration and mass separation, were delivered to the collinear laser spectroscopy station. The higher binding energy (and $\left.\left|\psi_{e}(0)\right|^{2}\right)$ of the $\mathrm{Be}^{+}$ion as compared to that of the $\mathrm{Li}$ atom ensures that measurements are only required to be precise to the order of $1 \mathrm{MHz}$. Nevertheless, for very light elements the systematic uncertainties associated with the measurement of the total acceleration voltage determine the overall systematic error which exceeds the perturbation caused by the nuclearvolume effect. As described in Section 3.4.1, a simultaneous co- and counter-propagating method of collinear laser spectroscopy was developed and permitted the measurement of the nuclear charge radii of ${ }^{7,9,10} \mathrm{Be}$, the one-neutron halo ${ }^{11} \mathrm{Be}$ and most recently, ${ }^{12} \mathrm{Be}[20,22]$.

The trend seen in the charge radii for the Be isotopes is close to that observed in lithium and the interpretation of results again invokes clusterization to explain the initial decrease towards ${ }^{10} \mathrm{Be}$. The motion of the centre-of-mass in the one-neutron halo isotope, ${ }^{11} \mathrm{Be}$, dominates the contribution to the increase in charge radius. Additionally, from the observed hyperfine structure of ${ }^{11} \mathrm{Be}$ the authors of [22] were able to confirm the magnetic moment previously measured using an optical pumping $\beta$ NMR experiment [338]. Fermionic molecular dynamics (FMD) calculations reproduced the charge radii however in [20] it was noted that the chosen effective interaction in the model failed to describe the parity inversion in the shell model orbits which gives the abnormal $1 / 2^{+}$spin-parity for the ${ }^{11}$ Be ground state. The latter reflects the breakdown of the $N=8$ shell closure with the $s_{1 / 2}$ halo orbit inverting with the $p_{1 / 2}$ orbit. ${ }^{12} \mathrm{Be}$, like ${ }^{11} \mathrm{Li}$, lies at the $N=8$ shell closure. With the two neutrons in ${ }^{12} \mathrm{Be}$ being more strongly bound, reflected in the decrease of the matter radius, it was therefore somewhat surprising to see an increase in the charge radius (Figure 10). This was understood as arising from strong mixing between the $p$ - and $s d$-shell orbits which lie very close in energy. According to FMD calculations, the $(s d)^{2}$ configuration contributes $\sim 70 \%$ to the ground state [20]. It is clear that the charge radius is thus very sensitive to mixing of states and can be used to validate the vanishing of the $N=8$ shell closure

The isotope shifts of ${ }^{7,9,11} \mathrm{Be}$ by Takamine $e t$ al were also investigated using the technique of opticaloptical double-resonance spectroscopy on the $2{ }^{2} S_{1 / 2} \rightarrow 2{ }^{2} P_{3 / 2}$ transition using laser-cooled ions captured in a linear Paul trap [24] (Section 3.6). The absolute resonance frequencies were determined however final results have yet to be published. Laser-microwave double-resonance (LMDR) spectroscopy was applied to the trapped and cooled $\mathrm{Be}^{+}$ions for precison measurements of the hyperfine structure of ${ }^{7,11} \mathrm{Be}[21,23]$. Assuming no hyperfine anomaly, the nuclear magnetic moment of ${ }^{7} \mathrm{Be}$ was determined to be $\mu\left({ }^{7} \mathrm{Be}\right)=-1.39928(2) \mu_{N}$ which agrees well with a simple shell model calculation [23]. In a similar manner, the nuclear magnetic moment of ${ }^{11} \mathrm{Be}$ was indirectly obtained from the measured ground state hyperfine structure constant and an average of the calculated differential hyperfine anomaly ${ }^{11} \Delta^{9}$ (Section 2.2.1), $\mu\left({ }^{11} \mathrm{Be}\right)=-1.68166(11) \mu_{N}$. This measurement is an important step in the study of the distribution of the halo neutron in ${ }^{11} \mathrm{Be}$ using a nuclear-model-independent optical probe. Naively, one may think of the charge radius of ${ }^{11} \mathrm{Be}$ represented by the core size, whereas the magnetization radius reflects the extension due to the halo neutron. This picture awaits confirmation through a more precise measurement of the magnetic moment in an experiment which will simultaneously measure the hyperfine constant [21].

\subsubsection{From proton halos to beyond the Island of Inversion (Ne to $\mathrm{Ti}$ )}

This region of the chart of the nuclides exhibits several different aspects of nuclear structure including proton halos, clustering and a weakening, almost to the extent of disappearance, of the traditional neutron magic numbers. A theoretical overview may be found in the work of Sorlin [353]. Of the 
elements studied via laser spectroscopy in this region the only one to cross the $N=8$ shell closure is Ne. Very sensitive detection was required for the short-lived isotopes, particularly for ${ }^{17} \mathrm{Ne}$, and therefore collinear laser spectroscopy was combined with state-selective collisional ionization and $\beta$-counting [27]. As this shell closure is approached it can be seen that the classical reduction in the rate of change of $\delta\left\langle r^{2}\right\rangle$ is observed (see Figure 10). The sharp increase below the $N=8$ shell closure for ${ }^{17} \mathrm{Ne}$ was first discussed in [28] and can be explained by the appearance of a two-proton halo structure [26]. Due to the sensitivity of the charge radius to the relative occupation of $s$ and $d$ orbitals, the observed increase was attributed to a proton $s^{2}$ admixture of $\sim 40 \%$. In general, the experimental values of both charge radii and one-neutron separation energies were remarkably well reproduced by FMD calculations [28]. To date this is the only proton halo candidate that has been studied via laser spectroscopy.

The neutron-rich side of stability in the vicinity of where the $N=20$ shell closure would be predicted has been the subject of extensive study for almost 40 years. The interest was first driven by the observation of unexpectedly large binding energies for ${ }^{31,32} \mathrm{Na}$ and ${ }^{31,32} \mathrm{Mg}$ in sharp contrast to the expected drop more commonly seen immediately following a shell closure [354, 355]. Laser spectroscopy measurements of the spins, moments and isotope shifts of neutron-rich Na isotopes supported the theory of a sudden onset of deformation [356]. This region, often referred to as the island of inversion is now characterised by nuclei with collective ground state properties caused by the inversion of the spherical ground state configurations with deformed intruder states with $n$-particle $-n$-hole excitations across the $N=20$ shell gap. The neighbouring isotopic chains of $\mathrm{Mg}$ and Ne have since been extensively studied at ISOLDE $[26-28,30]$ using a combination of laser spectroscopy and nuclear magnetic resonance techniques. This has extended the known region of shell breaking out to ${ }^{33} \mathrm{Mg}$ [357]. The proposed contributions to the inversion of the $n$-particle $-n$-hole states with respect to the 0 -particle -0 -hole states result in a reduction of the shell gap along with an increase in neutron-nucleon interactions [358] and monopole effects of the nuclear tensor force which is sensitive to a neutron excess [359]. To date, the aluminium chain has not been studied leaving a debate as to the full extent of this region.

As can be seen in Figure 1, little work has been carried out between the island of inversion and the next classical neutron shell closure at $N=28$. This arises mainly due to technical limitations such as the availability of the requisite beams as well as complex electronic structure. Mean-square charge radii of nuclei in the Ca region provide a coverage of the upper half of the $s d$ shell as well as the complete $\nu f_{7 / 2}$ shell. The extension of isotope shift and hyperfine structure measurements along the Ar chain from the $N=20$ shell closure to $N=28$ was reported in [36]. As the $f_{7 / 2}$ shell is gradually filled, the spectroscopic quadrupole moments change smoothly from negative to positive values, in agreement with shell model predictions. The evolution of the mean-square charge radii of the Ar isotopes follows a parabolic trend, symmetric around the mid-shell, on top of a monotonic increase as a function of neutron number. Superimposed on this is a pronounced "normal" odd-even staggering. The authors of [36] reproduce the experimental data using a simple shell model parameterization developed for the charge radii of $\mathrm{Ca}[360]$.

Recently, a series of measurements using bunched-beam collinear laser spectroscopy (described in Section 3.4.3) have been made on $\mathrm{K}$ and $\mathrm{Ca}$ isotopes, with the former resulting in investigations of the nuclear mean-square charge radii of all $f_{7 / 2^{-}}$and $\nu p_{3 / 2^{-}}$shell isotopes [39], and both providing new information on electromagnetic moments [38, 40, 45]. The ground state wavefunction of the odd- $A$ potassium isotopes is dominated by a single hole in the magic $Z=20$ shell. Measurement of the hyperfine structure of ${ }^{49,51} \mathrm{~K}$ firmly established a ground-state spin $I=1 / 2$ for ${ }^{49} \mathrm{~K}$ and $I=3 / 2$ for ${ }^{51} \mathrm{~K}$, indicating a "reinversion" of single-particle levels (seen for the first time) [38]. The Schmidt moments and effective single-nucleon $g$ factors of the relevant single-particle orbits in this region are very different from one another and were used to support the assignment of the ground-state spins. The complete isotopic chain between $N=19$ and $N=32$ is discussed in detail in [40]. Experimental results are compared with shellmodel calculations using the SDPF-NR and SDPG-U interactions showing an overall good agreement between the measured magnetic moments and calculations. Furthermore, ab initio calculations of open- 
shell nuclei have recently become possible in the Ca region (self-consistent Gorkov-Green's formalism) [361] and were used to probe the evolution of the proton effective single particle energies of the lowest $1 / 2^{+}$and $3 / 2^{+}$states. Although an overestimation of energy differences is seen (and is a general feature of calculated odd- $N$ spectra), the calculations reproduce the correct relative evolution which is extremely encouraging.

First measurements of the magnetic moments of ${ }^{49,51} \mathrm{Ca}$ and the quadrupole moments of ${ }^{47,49,51} \mathrm{Ca}$ have been reported [45]. The neutron-rich $\mathrm{Ca}$ isotopes have gained much attention in recent years in connection with the evolution of nuclear structure after the $N=28$ shell closure, with additional stabilized shell closures suggested at $N=32$ [362] and $N=34$ [363]. The measured $g$ factor of ${ }^{49} \mathrm{Ca}$, one neutron outside the doubly-magic ${ }^{48} \mathrm{Ca}$ isotope, is close to the effective single-particle value one would expect from the $p_{3 / 2}$ orbital. On the other hand, the value for ${ }^{51} \mathrm{Ca}$ indicates mixing with configurations due to neutron excitations across $N=32$, which would contradict the newly suggested closed-shell nature. Shell-model calculations assuming a rigid ${ }^{40} \mathrm{Ca}$ core agree well with the new magnetic moments, however disagree with isotopes in the vicinity of $N=20\left({ }^{41,43,45} \mathrm{Ca}\right)$, here indicating that excitations of nucleons across the $s d$ shell are important.

The evolution of the changes in the mean-square charge radii from Ar to Ti (Figure 10) displays a strong dependence on the atomic number $Z$. Such dramatic variations are not observed in the regions around $N=50,82$ and 126. As discussed above, the charge radii of Ar exhibit a rather monotonic increase as a function of $N$ up to the shell closure, similar to that of $\mathrm{K}$. This develops into the parabolic behaviour for $\mathrm{Ca}$ and continues into the anonymously downward-sloping trends in $\mathrm{Sc}$ and Ti. Of great importance will be the continued explorations of $\mathrm{Sc}$ and $\mathrm{Ti}$ isotopes towards and beyond the shell closures at $N=20$ and $N=28$. The new results for neutron-rich K isotopes above the $N=28$ shell closure closely follow the trend of neighbouring elements (for which data are available), at variance with the atypical $Z$ dependence below. This reflects the complex interplay of configurations as the protons and neutrons fill the same orbitals up to $N=28$. Above, charge radii are simply driven by a collective polarization of the proton distribution by the valence neutrons in the $p_{3 / 2}$ shell [39].

\section{Medium Mass nuclei}

Medium mass isotopes are considered here to be those which have atomic numbers from $Z=28$ to $Z=82$. At the low- $Z$ start of the region the atomic field shifts and mass shifts are comparable, both of order a few hundred $\mathrm{MHz}$ [364]. By the end of the region, field shifts, of order a few $\mathrm{GHz}$, are considerably larger than the mass effects and dominate the isotope shifts [364]. Experimentally, the size of the field shifts and precision required for their extraction has, at low- $Z$, required Doppler-reduced techniques and has been well suited to collinear laser spectroscopy. For the heavier, higher- $Z$, systems significant line broadenings can still be less than the observed hyperfine structures and isotope shifts and Doppler (or pressure) broadened spectra have successfully yielded useful nuclear data.

The motivation for studies of medium mass isotopes changes considerably over the extensive region. For the lightest systems, near $Z=30$, modern studies aim to investigate exotic, neutron-rich, shell and sub-shell closures pertinent to the nuclear r-process. At increasing- $Z$, on the approach to $Z \sim 40$, a rapidly changing region of nuclear structure is encountered and investigations here have concentrated on shape changes at the extremes of neutron excess and deficiency [76]. Experimental progress was historically hindered by the refractory nature of isotopes in this region - such difficulties have been overcome by 20 years of development of universal ion sources and laser desorption techniques. A notably uniform, parabolic, radial behaviour is observed around $Z \sim 50$ where measurements span the breadth of the nuclear chart approaching, or passing, $N \sim 50$ or $N \sim 82$ at their extremes [86, 89]. Recent experimental effort in this region concentrates at either extremity or is directed at isomeric systems in the chains. Toward higher $Z$, around 56, a wide range of exotic production from nuclear reactions (both 
fusion and fission) has permitted studies of the $N=82$ shell closure across the valley of stability, the $N=88$ shape change [101] and explorations of the structure of nuclei susceptible to octupolar vibrations and deformations [2]. Beyond the lanthanides, explored during pioneering laser ion source studies at St. Petersburg [103, 107, 110], another refractory range of open d-shell elements is encountered (and novelly studied using other than collinear techniques). Finally, a most famous region, and noteworthy series of measurements are found near $Z=80$ [2]. Presently measurements some 20 neutrons from stability, investigating shape trends beyond the $N=104$ mid-shell, can be found in the literature [146, 147]. The exploration of shape effects around the neutron mid-shell today affords data that stretches from minute staggering inversion in hafnium $[125,126]$, through transitional shape-changing systems in platinum [138] to the explosive, abrupt, shape changes famously observed in mercury.

Medium mass nuclei have been, and remain, explored by two principal techniques - collinear laser spectroscopy or resonance ionisation spectroscopy either within the primary ion source or utilising a secondary implantation site. Other important techniques, or variants on these spectroscopies, unique to individual facilities have been highlighted in Sections 3 and 4.

\subsection{Results and recent progress}

Here we review structural sub-regions through the medium mass region, highlighting the general nuclear structure investigated and concentrating on the most recent spectroscopy between $Z=28$ and $Z=82$. These, as outlined, are taken to be exotic neutron-rich nuclei near $Z=28$, the zirconium region, the tin region and a focus on medium-heavy elements at $N \sim 104$.

\subsubsection{Neutron-rich nuclei near $Z=28$}

At the start of the medium masses, $Z=28$, accessible isotopes of astrophysical interest have attracted spectroscopic attention with a recent focus on the migration of single-particle energies and level structures at the limits of stability (in particular at the extremes of neutron excess).

Long series of measurements along two isotope chains in the vicinity of ${ }_{28} \mathrm{Ni},{ }_{29} \mathrm{Cu}[61]$ and ${ }_{31} \mathrm{Ga}$ $[64,67]$, have recently been reported by the COLLAPS collaboration at IsOLDE. The spectroscopy exploited the ISCOOL cooler-buncher and the bunched-beam variant of collinear spectroscopy. For both systems measurements were made for neutron-deficient and neutron-rich isotopes, notably in the latter spanning the $N=40$ sub-shell and extending to $N=50-1$. The power of optical spectroscopy in indisputably assigning nuclear spins and multipole structures was critical to the work in which (surprising in ${ }_{31} \mathrm{Ga}$ ) changes in ground-state structures were for the first time detected and assigned. Experimental spectra recorded in the work are shown, for the neutron $g_{9 / 2}$ isotopes, in Figure 11 . The measured ground-state spin and magnetic moment of ${ }^{73} \mathrm{Ga}$ demonstrated a clear change of nuclear ground state and, novelly, place a lower limit on the energy of the first excited state, $75 \mathrm{eV}$ [67]. The spectroscopic quadrupole moments in ${ }_{29} \mathrm{Cu}$ [61] point to an $N=40$ sub-shell effect persisting in that chain but having an imperceptible effect on moments and radii in ${ }_{31} \mathrm{Ga}$. The measured charge radii display a pronounced change in isotonic character from these "shell-model systems" above $Z=28$ to those reflecting the strong collectivity associated with $N, Z \sim 40$ sub-shell in a matter of a few proton additions.

Recent measurements in ${ }_{29} \mathrm{Cu}$ have been complemented by two RIS studies [60, 62]. In this element, and similarly light cases, the selection of an optical transition in which a single valence s-electron configuration is encountered permits spectroscopy on the large hyperfine splitting arising from the Fermi contact field. Nuclear parameters, especially magnetic moments, are readily extractable when isotopically separated samples are studied even at modest resolution. The intercombination transition studied, $3 \mathrm{~d}^{10} 4 \mathrm{~s}^{2} S_{1 / 2} \rightarrow 3 \mathrm{~d}^{9} 4 \mathrm{~s} 4 \mathrm{p}{ }^{4} P_{1 / 2}$, displayed high sensitivity to the magnetic moment (and a substantial isotope shift) and measurements were possible for ${ }^{57,58,59,63,65} \mathrm{Cu}$. The reported magnetic 


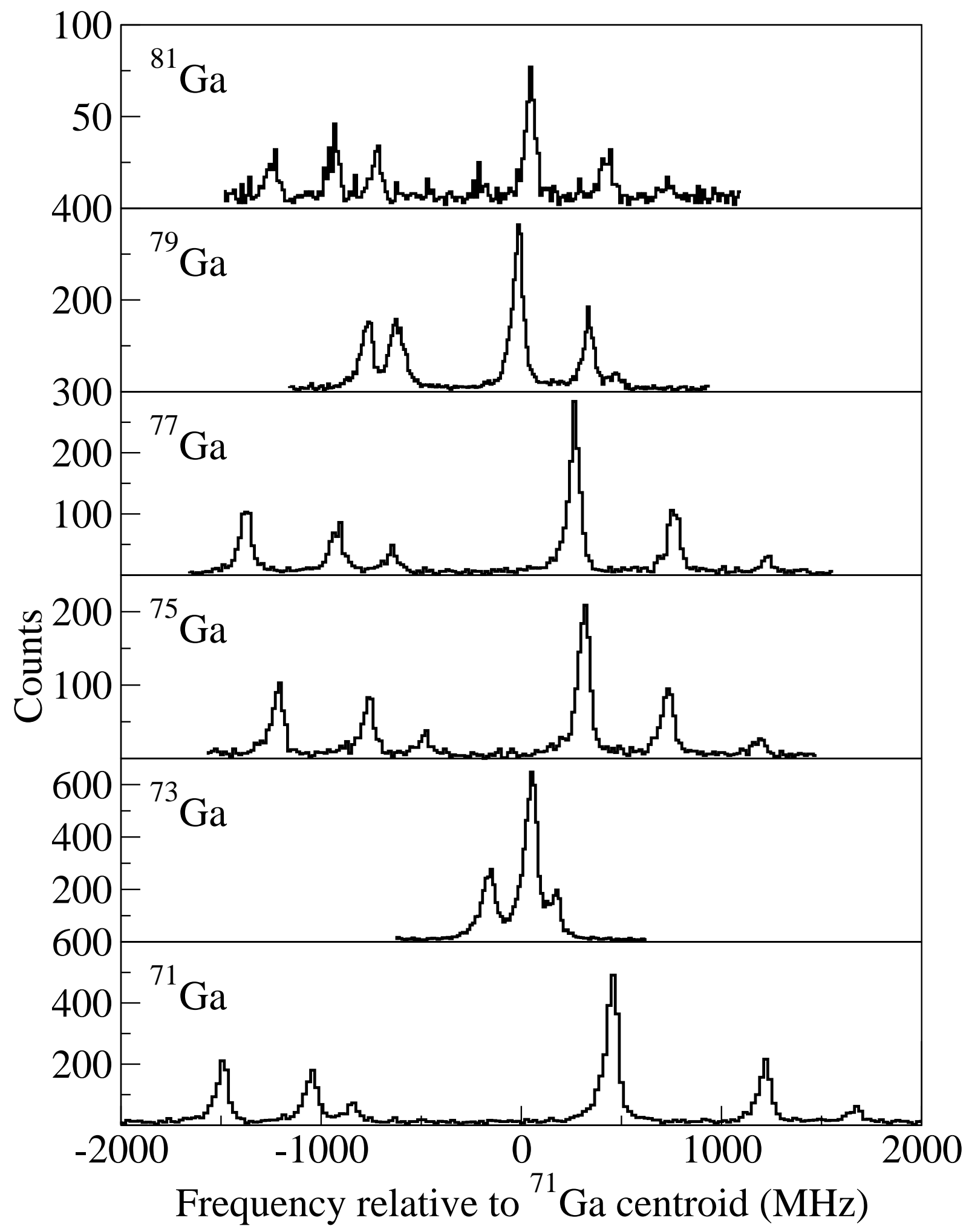

Figure 11: Select spectra of gallium isotopes recorded in work of reference [67]. The pronounced structural change at $A=73$ is clearly apparent. Figure kindly provided by B. Cheal. 
moments of ${ }^{57,58} \mathrm{Cu}$ attracted significant attention. The former strongly and surprisingly disagreed with a previous report [365]. The values of the moment for ${ }^{57,58} \mathrm{Cu}$ (with $N=28,29$ ) have profound implication for the nature of the $N=28$ shell closure in this isotope chain. The new moments reported in the work fit well with both phenomological estimates from mirror nuclei (the known ${ }^{57} \mathrm{Ni}$ isotope) and with shell model calculations based on the GXPF1 interaction [60]. A dramatic change in nature of the $N=28$ shell closure between $Z=28$ and $Z=29$ is not supported by the work.

Proposals to study the ${ }_{28} \mathrm{Ni}$ and ${ }_{30} \mathrm{Zn}$ systems at the ISOLDE facility (the latter being realised at the time of writing) will, on success, provide the even-even data critical for interpretation of the exotic nuclear moments and the first optical explorations of the $N=40$ sub-shell closure in these even- $Z$ chains.

\subsubsection{The zirconium region}

The rapidly changing nuclear structure in the zirconium region $(Z=40)$, whether with respect to nucleon addition, removal or excitation, has been of long standing interest in structure studies and a particular target for optical explorations [75, 78, 366].

A series of ${ }_{37} \mathrm{Rb}$ measurements [366] were the first to explore the charge radii and multipole structure of collective systems in the region and first to reveal the contrasting smooth onset of deformation in the neutron-deficient systems with the abrupt, $N=60$, shape change observed in the neutron-rich. The interest promoted the development of a range of spectroscopies, each first applied to the neighbouring ${ }_{38} \mathrm{Sr}$ chain and each still in experimental use today. The developments included non-optical (pumped population) detection [69], photon-ion(atom) coincidence spectroscopy [367, 368]and collinear multiple pumping strategies [369] and the reported efficiencies and sensitivities are still comparable to what has been achieved with the best variants of collinear spectroscopy to date. The achieved sensitivities reflect the favourable physical properties and low-density atomic (or ionic) structure found in the element strontium. The former affords efficient primary source production, the latter ensures optical transitions of high oscillator strength are available to spectroscopists. Study of higher- $Z$ isotopes, towards the peak of the collectivity in the region, were historically fatally hindered by the refractory nature of the open d-shell species and their complex, highly mixed, atomic structures. The application of ionguide technology was required to access native elemental (non-molecular) species [127]. The further application of in-flight Paul traps was then essential to cool both the thermal excitation of the isotopes and the spread of energies in the ionic ensemble as a whole [78]. Most recently in the example of ${ }_{39} \mathrm{Y}$ optical pumping within the Paul trap was further employed to provide spectroscopy away from ground state resonance lines [77].

In-cooler optical pumping [77] in the case of ionic yttrium enabled access to transitions other than the resonance lines from the $J=0$ ground state. Access to other transitions in this system were essential to provide sufficient, independent hyperfine structure splittings for a unique identification of $I, \mu, Q_{s}$ and $\left\langle r^{2}\right\rangle$ in each isotope and isomer. The demand for unique state identification was experimentally set by the observation, and mass measurement, of isomeric systems in the chain. The "complete" spectroscopy simultaneous mass and laser measurements provide is outlined in Section 8. When correctly assigned the measurements showed that the peak of deformation in the zirconium region occurs in the odd- $Z$ ${ }_{39} \mathrm{Y}$ chain and that the region is symmetric in the onset and loss of collectivity around this atomic number. In the region immediately before the $N=60$ shape change the yttrium isomer results provided spectroscopy in systems with $I>1 / 2$ (not found in the neighbouring $\mathrm{Sr}$ or $\mathrm{Zr}$ isotopes or in the odd-odd Y ground states) and facilitated access to measurements of the spectroscopic quadrupole moment. The quadrupole moments, before and after the shape change, show the $N=60$ shape change to truly be a transition to rigid prolate deformation but that this deformation softens rapidly either side of the transition. The region is shown in Figure 12 (with recent results at $Z=37$ and $Z=44$ ).

Figure 13 shows the two-neutron separation energies, $S_{2 n}$, extracted from precision mass measurements, in the zirconium region from $Z=36$ to $Z=44$ (right panel) and from $Z=20$ to $Z=28$ (left panel). 


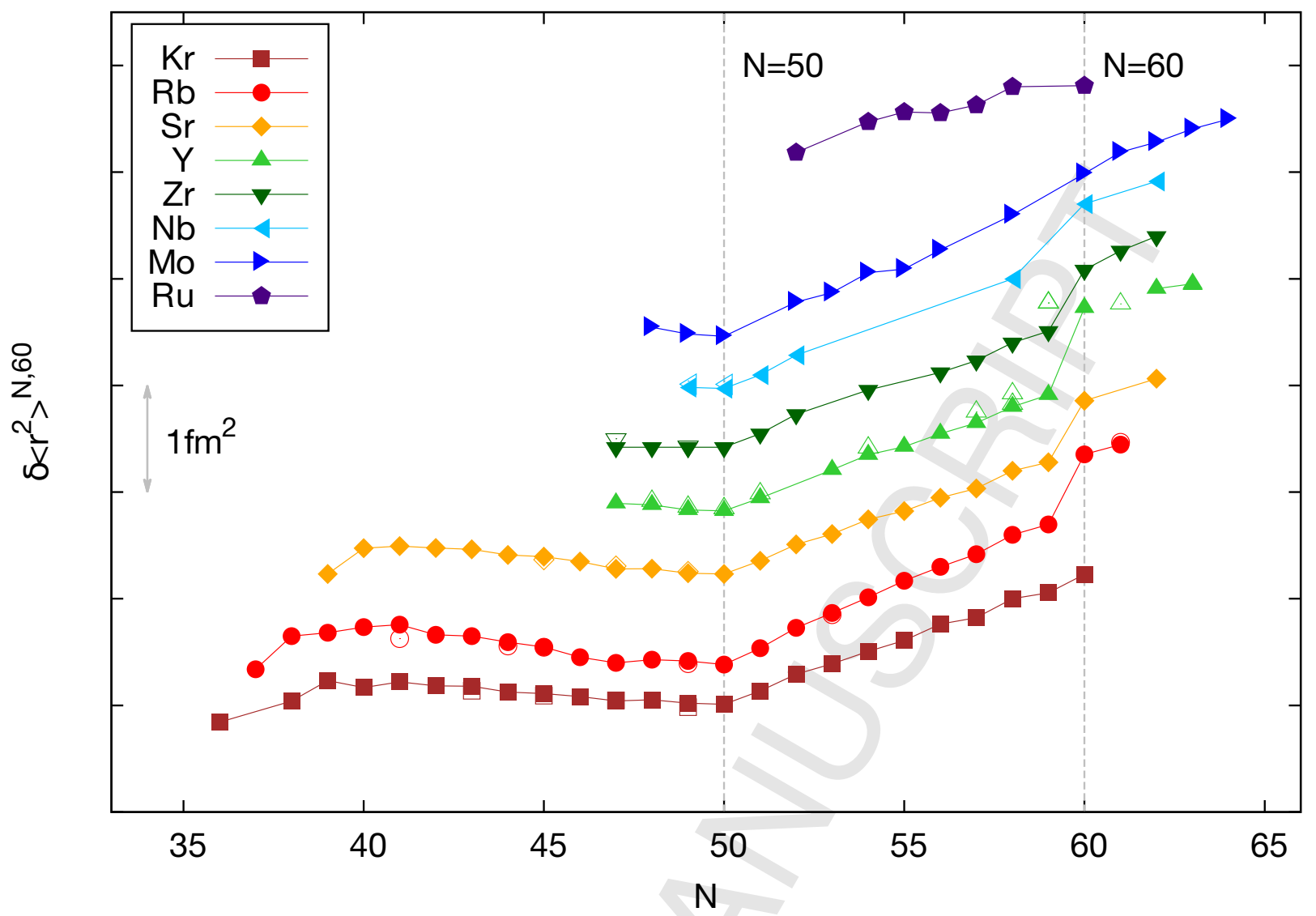

Figure 12: Changes in nuclear mean-square charge radii as a function of neutron number in the $\mathrm{Kr}$ to $\mathrm{Ru}$ region. All data have been obtained from references in Table 1. Isotope chains are relatively displaced by $0.6 \mathrm{fm}^{2}$ at $N=58$. Colour on-line.

As highlighted in reference [9], the trends in the two-neutron separation energies in the zirconium region show strong correlations to those of the charge radii. Near $N=60$ the onset of nuclear deformation notably perturbs the smooth progression of $S_{2 n}$ from that, for example, displayed by neutron-rich nuclei at lower $Z$ (shown in the right panel). The combination of the sensitivity of modern Penning-trap measurements and production capabilities at their parent facilities [9] result in $S_{2 n}$ measurements that extend significantly beyond the isotopes presently accessed by laser spectroscopy (both in $N$ and $Z$ ). The prospects for addressing structural investigations using combined mass and laser spectroscopy is returned to in Section 8.

Rapidly changing nuclear structure is observed in some of the most recent measurements in this region, at $Z=37$, but notably exploration of nuclear shape change was not the motivation for the study [71]. The $N=Z, I=0$ ground state in ${ }^{74} \mathrm{Rb}$ has a super-allowed $\beta$-decay to the ground state of ${ }^{74} \mathrm{Kr}$. The nuclear mean-square charge radius is a required input (or output) to the symmetry-breaking correction term used to evaluate (predict) the transition $\mathrm{ft}$-value. Such values can be used to explore the unitary nature of the Cabibbo-Kobayashi-Maskawa matrix or, if taken as unitary, to use other nuclear observables to infer the mean-square charge radius of ${ }^{74} \mathrm{Rb}$. The bunched beam spectroscopy reported in ref. [71] and result shown in Figure 12 confirms the prediction of a pronounced reduction in the mean-square charge radius of ${ }^{74} \mathrm{Rb}$ relative to a smooth extrapolation. 

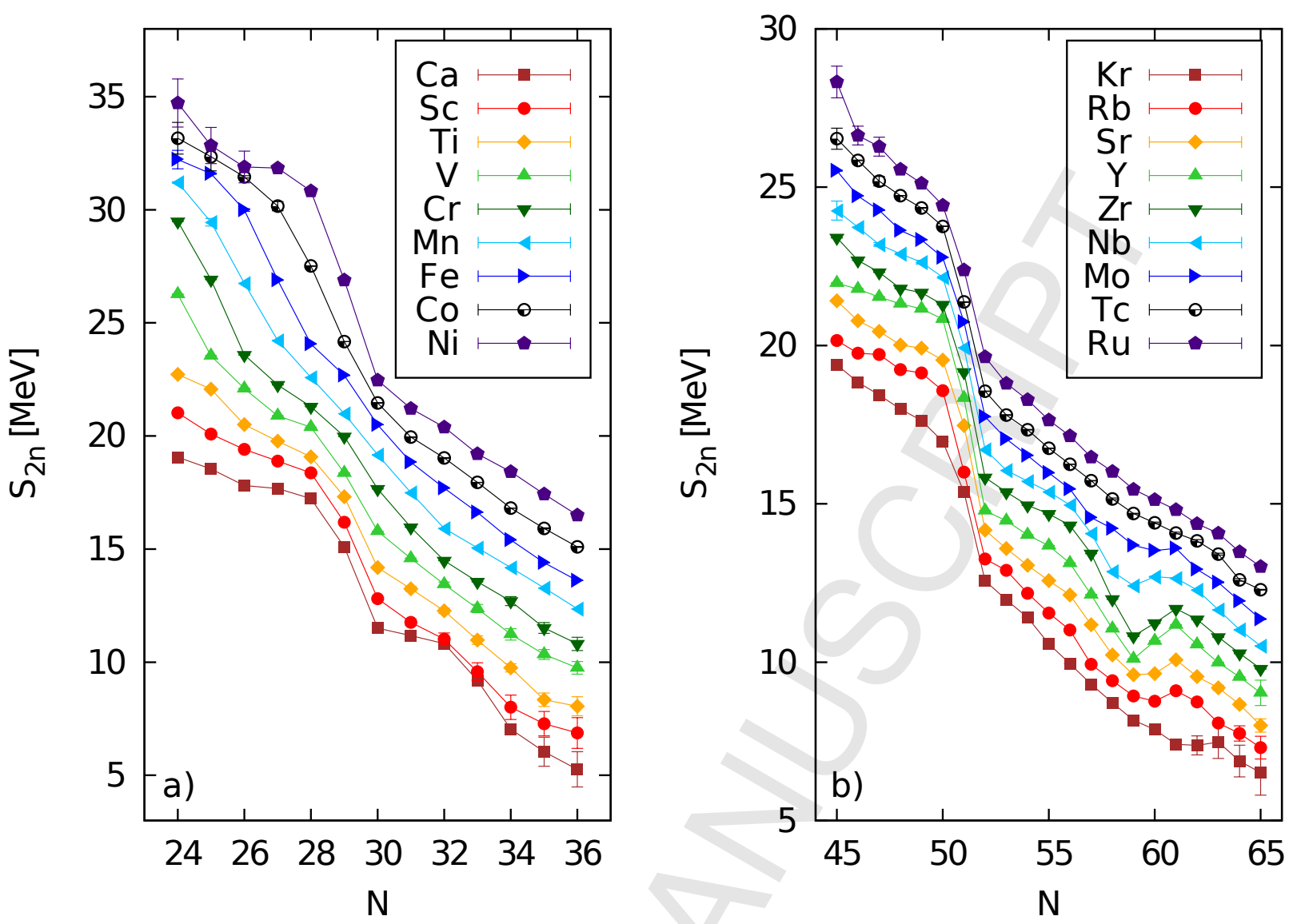

Figure 13: Experimental two-neutron separation energies in the vicinity of $Z=25$ (left panel) and $Z=40$ (right panel) as a function of neutron number. Shown are $S_{2 \mathrm{n}}$ values derived from the atomic massevaluation AME2012 [370]. Recent data from ISOLTRAP on neutron-rich isotopes of $\mathrm{Ca}$ [362] and Rb [371]. Colour on-line.

\subsubsection{The tin region}

Charge radii around ${ }_{50} \mathrm{Sn}$, illustrated in Fig. 14, display a degree of systematic and regular trends that persist throughout the major shell (spanning a space of 32 valence neutrons). The accessible isotopes, from $N<50$ to $N=82$ have radii that can be qualitatively, or quantitatively at the loss of strict physical interpretation, closely described by the simplest of models. A quadrupole contribution, proportional to the product of the number of particles and number of holes, and a constant odd-even staggering (OES) term added to a linearly increasing $\left\langle r^{2}\right\rangle$ describes the radial trend in neighbouring isotope chains almost precisely (using 3 or 4 variables). The defence of a linear and quadrupole term can be made in a variety of frameworks reflecting either a spherical-droplet contribution or single-particle orbital size and, for the latter, a residual interaction proportional to the number of valence pairings. The requirement of an additional odd-even contribution, that reduces the radius of odd- $N$ nuclei relative to the average of their even- $N$ neighbours, is clear from the data. This staggering term can be described theoretical, albeit for magic proton shells alone, in the models of Talmi [360] or blocked pair models of Zawischa et al. [372, 373].

The most recent work in the region has concentrated on explorations at the extremes of neutrondeficiency, at the $N=50$ shell closure in ${ }_{47} \mathrm{Ag}$ [86], and extremes of neutron-excess, at the $N=82$ shell closure in ${ }_{48} \mathrm{Cd}[89]$.

In the chain of cadmium, ${ }_{48} \mathrm{Cd}$, the spectroscopy accessed the most recently developed, sustained 


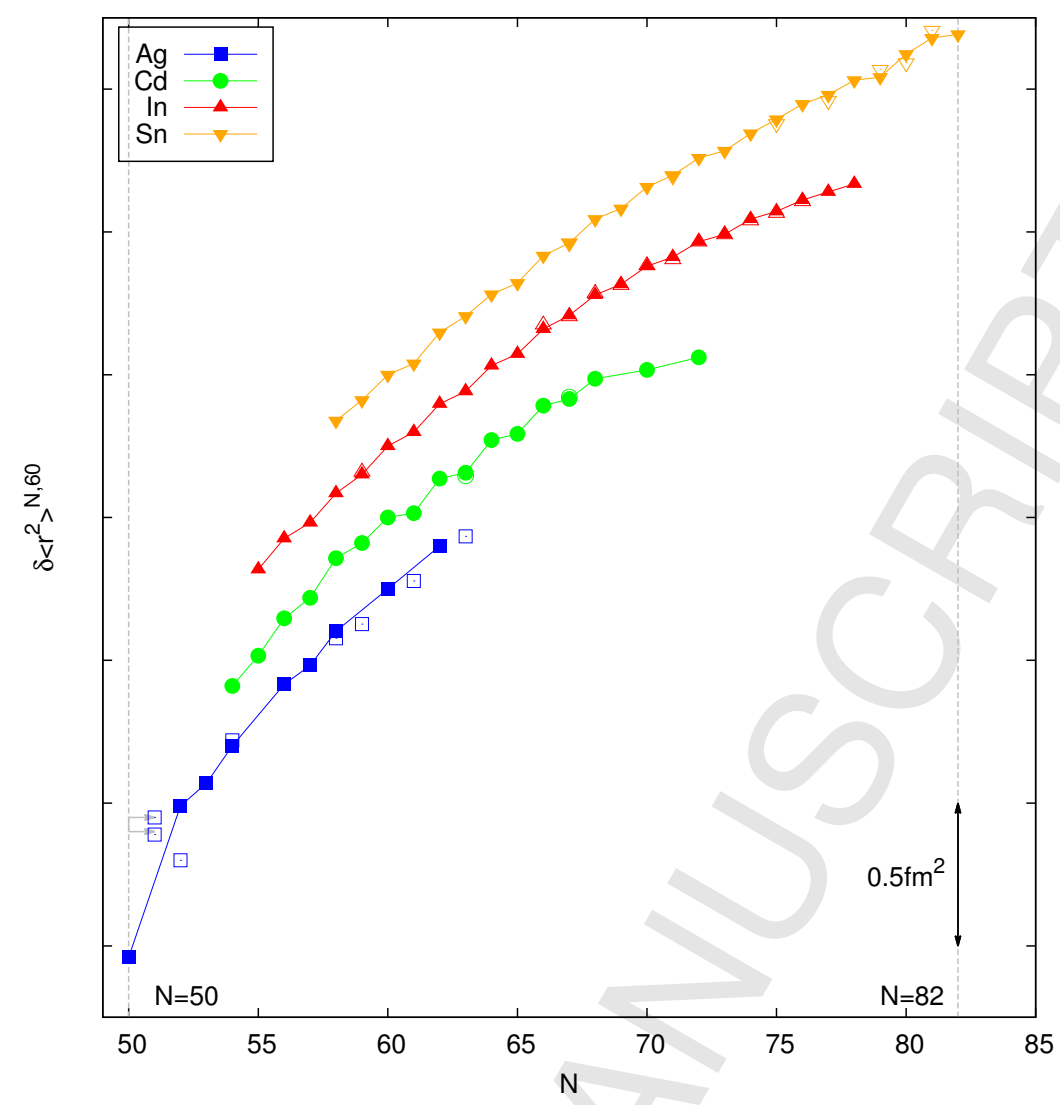

Figure 14: Changes in nuclear mean-square charge radii as a function of neutron number in the Ag to Sn region. All data have been obtained from references in Table 1. Light grey arrows indicated for the $\mathrm{Ag}$ isotopic chain represents different analysis options presented in [86]. Isotope chains have been offset by $0.5 \mathrm{fm}^{2}$. Colour on-line.

CW, short-wavelength UV production of 214.5-nm laser light. The short wavelength permitted excitation of the alkali-like ${ }^{2} S_{1 / 2}-{ }^{2} P_{3 / 2}$ D2 transition in the cadmium ion and access to a transition especially suitable for confident evaluation of critical atomic factors (field shifts, mass shifts, and hyperfine fields). The measured systems included the majority of the long-lived isomers abundant in the region and of these a near perfect linear trend of the $\frac{11}{2}^{-}$isomer radii is a notable result. For interpretation of this behaviour, a spherical single-particle shell model in a seniority coupling scheme can provide a convincing description for $h_{11 / 2}$ systems [89] - for the uniform behaviour to persist beyond this shell is a result still requiring further explanation.

The development of deep-UV laser frequencies such as that achieved for cadmium is critical to spectroscopy of elements lighter than $\mathrm{Ag}$ and for their homologues, ${ }_{42} \mathrm{Mo}-{ }_{46} \mathrm{Pd}$ and ${ }_{74} \mathrm{~W}-{ }_{78} \mathrm{Pt}$ and, when successfully made, will cover the majority of accessible elements in which laser spectroscopy of short-lived isotopes has yet to be realised (Section 8). The developments in the case of ${ }_{47} \mathrm{Ag}$ are comparably critical to future spectroscopy in the region. The $\mathrm{Ag}$ measurements at $N=50$ were made using a gas-cell technique, developed for further use at the forthcoming $\mathrm{S}^{3}$ facility, GANIL (Section 4.1.12). All late d-shell systems, highlighted above, are refractory and studies at the exotic shell closures requires an experimental sensitivity and selectivity beyond that of typical collinear techniques. 


\subsubsection{The mid-shell $(N \sim 104)$ platinum to lead nuclei}

At the end of the $Z=28-82$ region some of the earliest studied radioactive isotopes are located - the optical explorations of which predate laser spectroscopy $[2,51,206]$. The isotope shifts encountered are of order several $\mathrm{GHz}$ and equally sizable hyperfine structures are also observed. The large splittings, which permitted early spectroscopy, today allows line broadening techniques to be exploited but with the spectroscopy still providing precision nuclear data. The relaxation of resolution is, as common throughout optical spectroscopy, compromised to enhance spectroscopic sensitivity. In the ${ }_{78} \mathrm{Pt}$ to ${ }_{82} \mathrm{~Pb}$ region this sensitivity facilitates spectroscopy more than twenty isotopes from stability accessing and passing through the neutron mid-shell $(N=104)$ [142, 143, 146, 147]. The region, and that immediately above (7), has most recently benefited from on-going laser ion source and detection station developments notably at the ISOLDE facility. The refractory species were accessed during the work of the COMPLIS collaboration on laser desorption RIS (Section 3.5).

The heaviest isotopic chain ${ }_{82} \mathrm{~Pb}$ provides a datum standard to which both isotonic measurements and nuclear theoretical modelling can be anchored. The "kink" in the radial behaviour on crossing the magic shell closure has been inspected in numerous models, framework and force parametrisation (see for example ref. [374]). When viewed isotonically around $N=104$ the nuclear ground and isomeric states can be observed to display a shape transition region as striking as that seen in isotopes around $Z \sim 40$ both in the variety of shapes and structures and in their changes from one to another (the charge radii are shown in Figure 16 in 7). A remnant of the odd-even staggering, observed explosively in ${ }_{80} \mathrm{Hg}$ [206] persists through the isotones and remains apparent in a staggering inversion (the odd- $N$ nuclei having larger radii than the mean of the even- $N$ neighbours) as low as the mid-shell ${ }_{72} \mathrm{Hf}$ isotopes [125]. A longstanding desire in the field would see a reanalysis of these data, coupled to precision non-optical data, that permits an evaluation of isotonic radial trends (such as that attempted by Nadjakov et al. with existing data [375]). In order to reduce overwhelming systematic uncertainties such an extraction could only realistically be attempted following precision determination of absolute $\left\langle r^{2}\right\rangle$ that provide $\delta\left\langle r^{2}\right\rangle^{Z, Z^{\prime}}$ results at an accuracy comparable to those of isotope shifts. Potential routes to such measurements are highlighted in Section 8.

\section{Heavy Mass nuclei}

In the current review, the locality of heavy mass nuclei is defined as spanning those elements around $\mathrm{Pb}(Z=82)$ whose neutron-deficient isotopes exhibit the phenomenon of shape coexistence, to the very few nuclei studied above $\mathrm{U}(Z=92)$. The experiments reported in this region of the nuclear chart may broadly be attributed to the two general techniques discussed in sections 3.4 and 3.5, namely high resolution collinear laser spectroscopy as well as resonant ionisation spectroscopy (RIS). In recent years much activity has focused on developing these techniques further and indeed combining the attractive features of both in order to probe nuclei at the limits of stability.

An overview of optical measurements to date for this region is shown in Figure 15. Similar to the colour-coding of the full chart of nuclides (Figure 1) isotopes for which data are currently unpublished are shown in green, with additional radionuclides produced using laser resonance ionisation for radioactive beam production indicated in blue. One immediately notices the scarcity of information on nuclear ground-state properties from optical experiments above Ra corresponding to the last isotopic chain for which nuclear moments and mean-square charge radii are known from on-line experiments [376]. Such elements are not available at ISOL facilities and can only be produced by fusion reactions in heavy-ion collisions or via transfer reactions using radioactive targets. A combination of low production cross sections coupled with a lack of stable isotopes (thus few optical transitions available in literature) adds to the challenge of performing laser spectroscopy on the heaviest nuclei. 


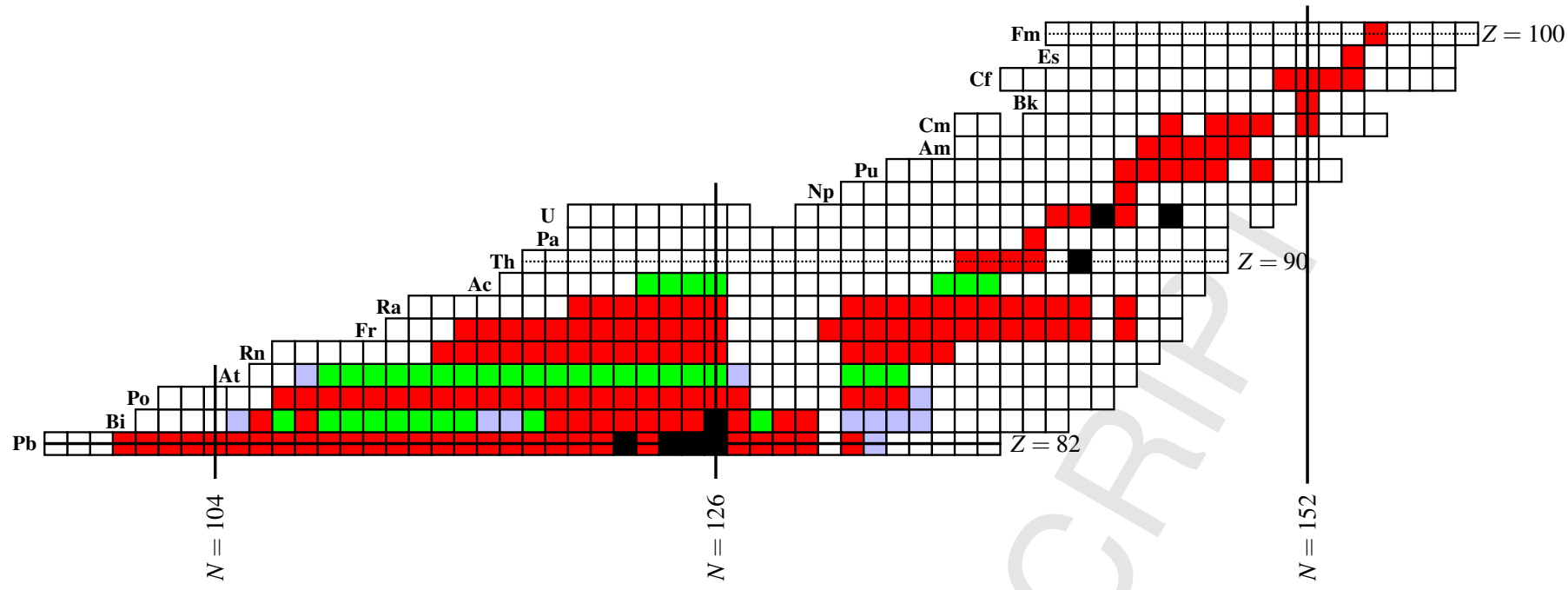

Figure 15: The heavy element region of the nuclear landscape above lead. Black squares indicate the stable or very long-lived nuclei, red squares indicate optical measurements of radioactive isotopes/isomers, green squares indicate nuclei for which the data are currently unpublished and blue squares represent radionuclides produced via methods of laser ionisation for radioactive ion beam experiments. Colour on-line.

\subsection{Results and recent progress}

\subsubsection{Shape coexistence in the lead region}

The phenomenon of nuclear shape coexistence arises due to the subtle interplay between collective and single-particle behaviour between nucleons. The region around $\mathrm{Pb}(Z=82)$, illustrated in Figure 16 , is an extremely well documented example, probed using a variety of experimental tools as well as hosting a number of theoretical explorations [377]. In the language of the nuclear shell model, shape coexistence may be obtained through multi-particle multi-hole excitations across known shell closures. On the other hand, self-consistent mean-field methods generate shape coexistence via competing configurations based on different nuclear shapes. The most spectacular example may be found precisely at the middle of two closed shells $(N=82$ and $N=126)$ in ${ }^{186} \mathrm{~Pb}$, whereby configurations based on $2 p$ - $2 h$ and $4 p$ - $4 h$ excitations across the $Z=82$ shell gap result in a coexistence of a spherical ground state and two excited $0^{+}$states corresponding to oblate and prolate deformed shapes at an energy of approximately $1 \mathrm{MeV}$ [378].

The transitional region extending from the rare-earth nuclei to the lead isotopes has provided a wealth of experimental information. One of the early highlights was the discovery of shape coexistence in the neutron-deficient $\mathrm{Hg}(Z=80)$ isotopes from optical hyperfine structure studies, with an extremely large reversed odd-even staggering (OES) evident in the charge radii below $N=106$ [379]. Subsequent studies using pulsed laser-induced desorption RIS on neutron-deficient isotopes of $\operatorname{Ir}(Z=77), \operatorname{Pt}(Z=78)$ and $\mathrm{Au}(Z=79)$ charted the changes in mean-square charge radii down to ${ }^{182} \mathrm{Ir},{ }^{178} \mathrm{Pt}$ and ${ }^{183} \mathrm{Au}$, respectively $[136,138,141]$. The main feature seen in the Ir isotopic chain is the sudden increase in the mean-square charge radius between $N=110$ and $N=109$, associated with a transition from a gamma-soft regime to an axially symmetric prolate shape. In a similar manner, the Au chain also exhibits a single jump but at a different neutron number. Below ${ }^{186} \mathrm{Au}$ no staggering has been observed suggesting that all Au nuclei with $A \leq 186$ are strongly prolate-deformed in the ground states. The Pt chain reflects a region rich in shape instabilities with large differences in the charge radius of the ground and isomeric states for ${ }^{183,185} \mathrm{Pt}$, a rapid reduction in the deformation observed at $A=178$ and a relatively strong 
inverted OES below $A=188$ indicating a deformation change between the even-even (triaxial) and the odd-even (prolate) isotopes.

The advent of hot cavity in-source RIS has allowed further elucidation of this phenomenon by expanding the studies to the $\mathrm{Tl}(Z=81), \mathrm{Pb}, \mathrm{Bi}(Z=83)$ and $\mathrm{Po}(Z=84)$ isotope chains $[88,142,146,151]$. The change in mean-square charge radii of $\mathrm{Pb}$ has been extended down to ${ }^{182} \mathrm{~Pb}$. Unlike the neighbouring $\mathrm{Hg}$ isotopes, as the neutron mid-shell at $N=104$ is reached and crossed the ground-state wavefunction of $\mathrm{Pb}$ remains essentially spherical. A small deviation from spherical droplet model predictions was observed below ${ }^{196} \mathrm{~Pb}$ which earlier was thought to indicate the presence of deformation as the $0^{+}$ intruder state approaches the ground state. However, modern theoretical calculations indicate that the experimental data are very sensitive to correlations in the ground-state wavefunctions. The deviation from the droplet model can therefore be explained without the introduction of static deformation. Recent studies of $\mathrm{Tl}$ indicate a close resemblance to the $\mathrm{Pb}$ isotopic chain, indicating no strong oddeven staggering in the ground states and a near-spherical shape persisting beyond the mid-shell to the lightest isotope studied, ${ }^{183} \mathrm{Tl}$. It was expected due to symmetry arguments that the neutron-deficient Po isotopes would show a similar behaviour in the charge radii to $\mathrm{Hg}$ and thus it was a surprise when a sudden departure from sphericity was observed starting at $N=114\left({ }^{198} \mathrm{Po}\right)$, occurring at larger neutron numbers than in the $Z \leq 82$ isotones. This departure from sphericity is only partially reproduced with beyond mean-field calculations and clearly indicates a strong onset of collectivity at $N=114$. Recent results obtained for isotopes of At and Au to further elucidate the understanding of shape coexistence in the $\mathrm{Pb}$ region have yet to be reported. An important goal for future studies would be to extend the charge radii of the most neutron-deficient radon and radium nuclei to clarify this picture.

Very recently, in the first physics application of the LIST (Section 3.5.1), high purity neutron-rich Po beams have been provided by suppressing the strong contamination from surface-ionized Fr by more than three orders of magnitude. This contamination prevented the possibility to perform in-source spectroscopy on a number of Po isotopes in earlier studies. Now, for the first time, RIS has been performed directly inside the LIST device allowing the study of the hyperfine structure of ${ }^{217}$ Po as well as a nuclear decay spectroscopy study of ${ }^{219} \mathrm{Po}$ [154]. Future access to RIS of ${ }^{211,212} \mathrm{Po}$ will require further optimisation of the LIST performance due to the high production rate and relatively poor suppression of ${ }^{212} \mathrm{Fr}$.

By comparing relative changes in mean-square charge radii (or indeed isotopic shifts under the assumption of a negligible mass shift) one is able to identify similarities or discrepancies between isotopic chains. This formalism was originally introduced by Hull and Stroke who observed marked similarities in the relative isotopic shifts for isotones in $\mathrm{Hg}$ and $\mathrm{Tl}$ [383]. Later, such comparisons were extended above the $Z=82$ shell closure by Barboza-Flores [384], Kowalewska [155], Campbell [385] and Pearson [150]. It is remarkable that within the region near stability, an incredible reproduction of small scale structure such as odd-even staggering is reproduced within errors. Such behaviour, although unlike any other region of the nuclear chart, is characteristic of the region near $N=126$ and above $Z=82$. Departures from this almost identical nature are seen below $N=119$ as the deformed region is approached. Below the proton shell closure such level of correspondence is much weaker and thus it appears that the $\pi h_{9 / 2}$ valence protons outside the $\mathrm{Pb}$ core are inert spectators, whereas the proton holes of $\mathrm{Tl}$ and $\mathrm{Hg}$ have much more influence on the details of the charge radii.

\subsubsection{The $N=126$ shell closure and above}

The shell closure at $N=126$ manifests itself in a characteristic change in the slope (kink) in the charge radii as can be seen in Figure 16. This behaviour is observed at all near-stability shell closures with the magnitude of the gradient change decreasing steadily with the $Z$ of the magic shell. Theoretically, original Skyrme parameterizations were unable to account for the isotope shift in the $\mathrm{Pb}$ region whereas the relativistic mean-field (RMF) model appeared to have success. In the 1990s, the authors of [386] 


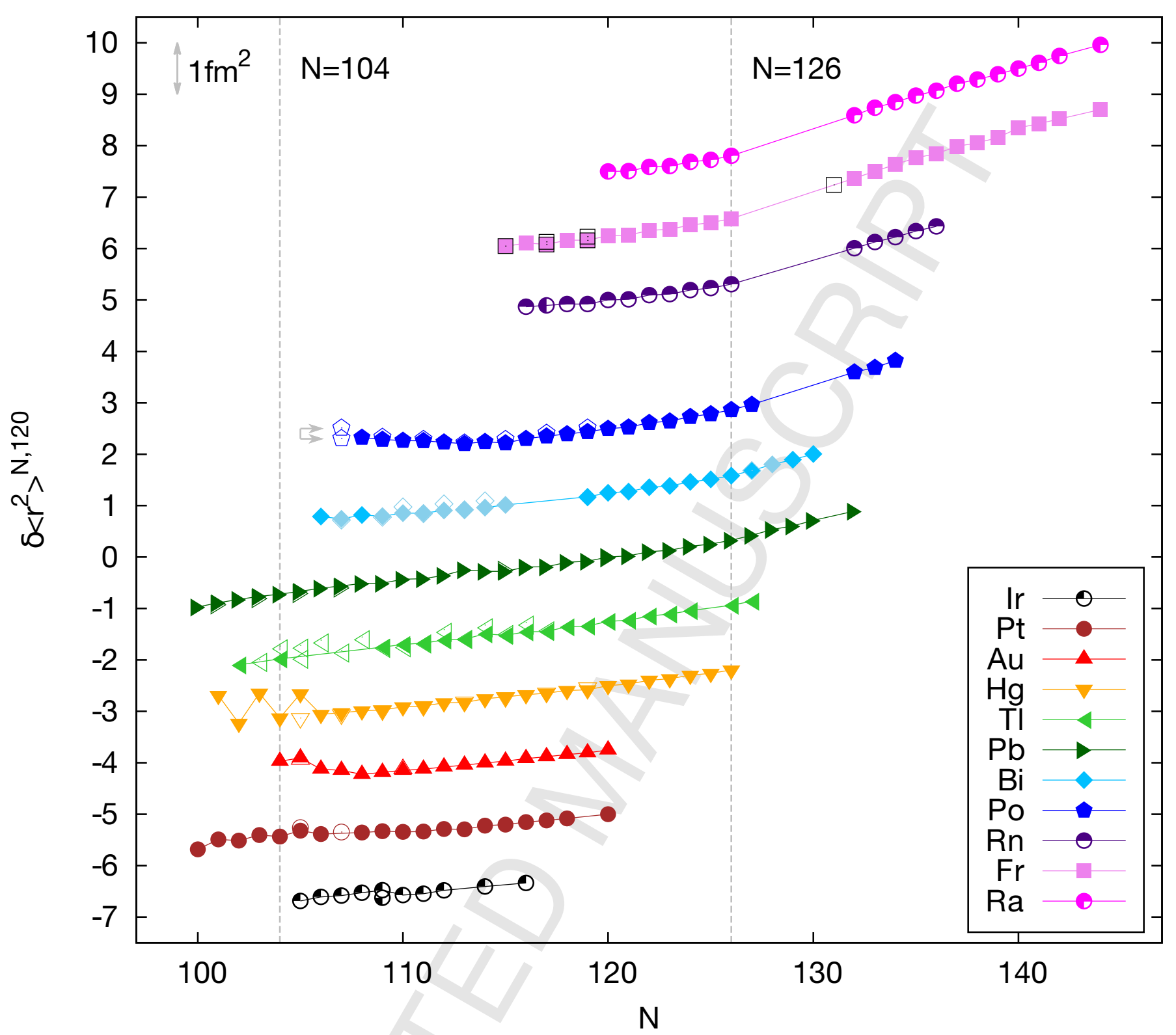

Figure 16: Changes in nuclear mean-square charge radii as a function of neutron number in the Ir to Ra region, relative to $N=120$ (apart from Ir). All data have been obtained from references in Table 1 (the re-evaluated analysis of the Ra data [166] are used) apart from unpublished measurements in Bi, obtained at the IRIS facility, Gatchina, highlighted in light blue, kindly provided by A. Barzakh. The isotope chains have been arbitrarily offset by $1 \mathrm{fm}^{2}$ from one another for clarity. Isotope shift values for Bi have been evaluated using the atomic factors from [380] and for Po using the method described in [151]. Using the King plot method, francium isotope shift data from [242, 381] and atomic factors for the 718-nm transition from [382] have been used to extract values for the atomic factors of the 422-nm transition. Using these factors, the isotope shift values from [158], [159] and [160] have been transferred to the 718-nm transition. This transferred data have been combined with Coc et al [381] and Voss et al [157] using atomic factors from Dzuba et al [382] for re-evaluation. Grey arrows indicated for the Po isotopic chain represents different analysis options presented in the corresponding references. Open symbols represent isomeric states. Colour on-line. 
analyzed the differences between these two approaches and by modifying the spin-orbit contribution to the Skyrme-Hartree-Fock parameterization, the kink at $N=126$ could be reproduced. More recent work by Goddard et al indicates that the occupation of the $1 i_{11 / 2}$ neutron orbital beyond $N=126$ overlaps strongly with the proton orbitals with the resulting effect reflected in the kink which can be reproduced in both $\mathrm{Pb}$ and $\mathrm{Po}$ chains [374].

The trends in the charge radii of $\operatorname{Rn}(Z=86), \operatorname{Ra}(Z=88)$ and the neutron-rich isotopes of $\operatorname{Fr}(Z=87)$, all performed by collinear fast-beam laser spectroscopy, have been discussed in earlier reviews $[2,3]$. Recently, a combined analysis of the available isotope-shift data from optical spectra of Ra atoms and $\mathrm{Ra}^{+}$ions has been performed by Wansbeek et al [166], extending the earlier analysis of the ISOLDE collaboration [376]. Among others, more accurate ab initio values for the field-shift constants are used and more recent and extended isotope-shift data have been included. These re-evaluated data have been compared with changes in mean-square charge radii of neutron-rich Fr isotopes obtained using the newly installed CRIS beam line at ISOLDE, indicating a very similar trend along the whole mass chain [160]. These isotopes lie at the border of the region associated with reflection-asymmetric shapes. Theoretically, the nature of the charge radii of reflection-asymmetric nuclei has been addressed using macroscopic-microscopic mass models. In the framework of the Finite-Range Droplet Model, Iimura and Buchinger illustrate a better agreement with experimental charge radii data when they include the octupole degree of freedom in comparison to simulations performed without reflection asymmetry [387].

The Fr isotopic chain has also been probed towards exotic neutron-deficient isotopes, covering the mass range previously studied by Coc et al (from $N=126$ to $N=120$ ) [381] which now extends down to ${ }^{202} \mathrm{Fr}[157,158]$. Voss and collaborators developed the most recent extension of collinear laser spectroscopy, using high-frequency intensity modulation of CW laser light to suppress the effect of hyperfine pumping [157]. This enhances the intensity of weaker components and directly assists in the determination of nuclear spins. All isotopic chains from $\mathrm{Pb}$ to $\mathrm{Ra}$ (Figure 16) exhibit a normal odd-even staggering (OES) down to $N=119$, an effect which is more strongly pronounced for Fr than for Pb. This indicates an increase in pairing energy in the even mass Fr nuclei and marks a loss of pure single-particle structure [157]. The most exotic isotope of Fr, ${ }^{202} \mathrm{Fr}$, was studied with the technique of collinear resonance ionisation spectroscopy. Flanagan et al also identify the close correlation between the trend of OES in the $\mathrm{Pb}$ and Fr chains, noting a departure from this trend at ${ }^{203} \mathrm{Fr}$ which suggests an earlier onset of collectivity than in the $\mathrm{Po}$ or $\mathrm{Pb}$ chains. Future work should aim to extend the Fr chain towards ${ }^{199} \mathrm{Fr}$ where an intruder state has been reported to invert with the ground state leading to an onset of deformation $[388,389]$. In parallel, theoretical efforts are required to elucidate the collective contributions to the ground state wave function in a similar manner as performed on the $\mathrm{Pb}$ and $\mathrm{Po}$ nuclei.

\subsubsection{Laser spectroscopy of the actinide elements and beyond}

The actinide elements cover the range of atomic numbers from $\operatorname{Ac}(Z=89)$ to $\operatorname{Lr}(Z=103)$, beyond which lie the superheavy elements. Traditionally, the main interest in actinide spectroscopy has been in energy level analysis, resulting in the construction of large data banks of atomic levels and transitions which represent a starting point for exploring the complex structure associated with the actinides. Conventional emission spectroscopy performed on Ac to Es has been investigated experimentally and theoretically, and rather good spectroscopic information is now available. For a complete overview of actinide spectra and electronic structures the reader is referred to Worden et al [186].

One of the most fundamental quantities of an element is the first ionisation potential (IP). A precise determination of the IP is important for the identification of systematic trends in binding energies as well as providing information concerning atomic structure. A variety of laser spectroscopic techniques have been used to precisely determine the first ionisation potential (IP) from Th to Es, with the exception of $\mathrm{Pa}$ [186]. For the common lighter actinides, the most accurate values for the IP were obtained through the analysis of converging Rydberg series. Later, resonance ionisation mass spectrometry (RIMS), first 
developed for ultra-trace analysis of the actinides, was applied and allowed an accurate determination of the IPs of Ac, Am, Cm, Bk, Cf and Es with samples sizes of only $10^{12}$ atoms. Most recently RIMS has been used to significantly refine the first IP of Ac, a radioactive element with only one long-lived isotope, ${ }^{227} \mathrm{Ac}\left(\tau_{1 / 2}=28 \mathrm{yr}\right)$, using samples sizes as small as $5 \times 10^{11}$ atoms [390]. An attempt to determine the first IP of Fm using ${ }^{255} \mathrm{Fm}\left(\tau_{1 / 2}=20.1 \mathrm{~h}\right)$ failed due to the short half-life as well as a lack of spectroscopic data. That work did however result in the first ever observation of two atomic transitions [283]. Recently, Wendt et al have performed a study on the systematic trends of the first IPs for both the lanthanide and actinide elements in order to make better predictions for the transactinides [391].

In general, the scarcity of ground state nuclear structure information above Ra from optical measurements is apparent and is summarised in Table 4. We note that isotopes of Ac have yet to be published and the work of Edelstein [392] on ${ }^{249} \mathrm{Cf}$ is non-optical, thus do not appear in Table 1. Isotopes of Th and $\mathrm{U}$ were originally targeted to extend the experimental information gained in the $\mathrm{Rn}$ and $\mathrm{Ra}$ chains with the purpose of contributing to an understanding of the static octupole deformation associated with this region [170, 173]. Laser spectroscopy of ${ }^{229} \mathrm{Th}$ is currently being pursued by many groups around the world due to a unique low-lying isomeric state lying within several $\mathrm{eV}$ of the ground state. This anomalously small energy separation is predicted to be within the range of modern lasers which opens a number of exciting possibilities including the development of a "nuclear" clock, a laboratory for testing the stability of fundamental constants, to the realisation of a novel nuclear-based laser. With the direct observation of the decay of the isomer still pending, an unambiguous signature to prove the existence of the state would be evidence of hyperfine components which cannot be attributed to the nuclear ground state $[167,168]$. The extensive work by Backe and colleagues using the RADRIS method culminated in the successful measurements of isotope shifts for superdeformed Am fission isomers ${ }^{240 f, 242 f, 244 f} \mathrm{Am}$ $[183,184]$. The results demonstrated the stability of the deformation in the second potential minimum as neutron pairs are added.

Table 4: Ground state nuclear structure information from Ac $(Z=89)$ to $\operatorname{Fm}(Z=100)$. An empty field indicates that the corresponding quantity is unknown. Nuclear moments highlighted with $\mathrm{a} \times$ are found in Stone [7], whereas $\bigcirc$ indicates that the corresponding parameter may be found in the references. Measurements yielding isotope shift information are represented by $\Delta$. All nuclear spin $I$ information has been taken from [393] with assignments in brackets considered to be tentative.

\begin{tabular}{c|c|c|c|c|c|c|c|l}
\hline \hline Element & $Z$ & $N$ & $A$ & $I$ & $\mu$ & $Q$ & IS & Reference(s) \\
\hline Ac & 89 & 123 & 212 & & & & & {$[291]$} \\
& & 124 & 213 & & & & & {$[291]$} \\
& & 125 & 214 & $(5+)$ & & & & {$[291]$} \\
& & 126 & 215 & $9 / 2^{-}$ & & & & {$[291]$} \\
& & 136 & 225 & $\left(3 / 2^{-}\right)$ & & & & {$[394]$} \\
& & 138 & 227 & $3 / 2^{-}$ & $\times$ & $\times$ & & {$[281]$} \\
\hline Th & 90 & 137 & 227 & $1 / 2^{+}$ & & & $\Delta$ & {$[170]$} \\
& & 138 & 228 & $0^{+}$ & & & $\Delta$ & {$[168,170]$} \\
& & 139 & 229 & $5 / 2^{+}$ & $\times$ & $\bigcirc$ & $\Delta$ & {$[167-170]$} \\
& & 140 & 230 & $0^{+}$ & & & $\Delta$ & {$[168,170]$} \\
\hline $\mathrm{Pa}$ & 91 & 142 & 232 & $0^{+}$ & & & $\Delta$ & {$[168,170]$} \\
\hline $\mathrm{U}$ & 92 & 141 & 231 & $3 / 2^{-}$ & $\times$ & & & {$[171]$} \\
\hline \hline
\end{tabular}


Table 4 - continued from previous page

\begin{tabular}{|c|c|c|c|c|c|c|c|c|}
\hline Element & $Z$ & $N$ & $A$ & $I$ & $\mu$ & $Q$ & IS & Reference(s) \\
\hline & & 142 & 234 & $0^{+}$ & & & $\Delta$ & {$[172,173]$} \\
\hline & & 143 & 235 & $7 / 2^{-}$ & $x$ & $x$ & $\Delta$ & {$[172-174]$} \\
\hline & & 144 & 236 & $0^{+}$ & & & $\Delta$ & {$[172,173]$} \\
\hline & & 146 & 238 & $0^{+}$ & & & $\Delta$ & {$[172,173]$} \\
\hline $\mathrm{Np}$ & 93 & 144 & 237 & $5 / 2^{+}$ & $x$ & $x$ & & {$[175]$} \\
\hline $\mathrm{Pu}$ & 94 & 144 & 238 & $0^{+}$ & & & $\Delta$ & {$[176]$} \\
\hline & & 145 & 239 & $1 / 2^{+}$ & $x$ & & $\Delta$ & {$[176]$} \\
\hline & & 146 & 240 & $0^{+}$ & & & $\Delta$ & {$[176]$} \\
\hline & & 147 & 241 & $5 / 2^{+}$ & $x$ & $x$ & $\Delta$ & {$[176]$} \\
\hline & & 148 & 242 & $0^{+}$ & & & $\Delta$ & {$[176]$} \\
\hline & & 150 & 244 & $0^{+}$ & & & $\Delta$ & {$[176]$} \\
\hline $\mathrm{Am}$ & 95 & 145 & $240 \mathrm{~m}$ & & & 0 & $\Delta$ & {$[178-180,183,184]$} \\
\hline & & 145 & 240 & $\left(3^{-}\right)$ & & & $\Delta$ & [178] \\
\hline & & 146 & 241 & $5 / 2^{-}$ & $x$ & $x$ & $\Delta$ & {$[177,178,180,181,183,184]$} \\
\hline & & 147 & $242 \mathrm{~m}$ & $5^{-}$ & $x$ & $x$ & $\Delta$ & {$[182-184]$} \\
\hline & & 148 & 243 & $5 / 2^{-}$ & $\times$ & $x$ & $\Delta$ & {$[177,178,180,182,183]$} \\
\hline & & 150 & $244 \mathrm{~m}$ & & & & $\Delta$ & [184] \\
\hline $\mathrm{Cm}$ & 96 & 146 & 242 & $0^{+}$ & & & $\Delta$ & {$[185,186]$} \\
\hline & & 148 & 244 & $0^{+}$ & & & $\Delta$ & {$[185,186]$} \\
\hline & & 149 & 245 & $7 / 2^{+}$ & $x$ & & $\Delta$ & {$[185,186]$} \\
\hline & & 150 & 246 & $0^{+}$ & & & $\Delta$ & {$[185,186]$} \\
\hline & & 152 & 248 & $0^{+}$ & 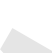 & 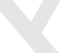 & $\Delta$ & {$[185,186]$} \\
\hline $\mathrm{Bk}$ & 97 & 152 & 249 & $7 / 2^{+}$ & $x$ & 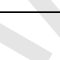 & & {$[186,187]$} \\
\hline $\mathrm{Cf}$ & 98 & 151 & 249 & $9 / 2^{-}$ & 0 & $\bar{s}$ & & {$[188,392]$} \\
\hline & & 152 & 250 & $0^{+}$ & + & & & [188] \\
\hline & & 153 & 251 & $1 / 2^{+}$ & & & & [188] \\
\hline & & 154 & 252 & $0^{+}$ & & & & [188] \\
\hline Es & 99 & 154 & 253 & $7 / 2^{+}$ & $x$ & $x$ & & {$[189,190]$} \\
\hline $\mathrm{Fm}$ & 100 & 155 & 255 & $7 / 2^{+}$ & 0 & 0 & & [191] \\
\hline
\end{tabular}

The impact of relativistic- and QED-effects on the atomic structure gain in importance going to heavier elements, even influencing binding energies of the valence electrons and thus chemical properties. In preparation for searches for atomic resonances on the transfermium elements $(Z>100)$, calculations for No $(Z=102)$ and $\operatorname{Lr}(Z=103)$ have been performed in order to constrain the spectral regions [395397]. An experimental determination of the IP would therefore constitute a valuable test of modern Multi-Configuration Dirac-Fock (MCDF) and relativistic coupled-cluster (RCC) calculations.

In order to access spectroscopic information of such elements, the very high sensitivity of techniques such as RADRIS will be required. Such elements may only be synthesised at on-line facilities (with correspondingly very low production rates), and are characterised by short lifetimes and a complete lack of information on atomic excitation schemes required for laser spectroscopy. A research programme has been initiated at the velocity filter SHIP at GSI to investigate the alpha-emitting element No $(Z=102)$. The first aim is the search for unknown atomic energy levels and, if successful, a direct determination of the ionisation potential. With a half-life of $55 \mathrm{~s},{ }^{254}$ No can be produced by the fusion-evaporation reaction ${ }^{208} \mathrm{~Pb}\left({ }^{48} \mathrm{Ca}, 2 \mathrm{n}\right){ }^{254} \mathrm{No}$ with a production cross section of $\sigma \sim 2 \mu \mathrm{b}$ [398]. With production rates of a few atoms per second, the quality of theoretical predictions for an atomic level search with pulsed lasers is crucially important. In a first experimental run behind the velocity filter SHIP at GSI, Laatiaoui and collaborators have applied the RADRIS method for an atomic level search of No using a two-step 


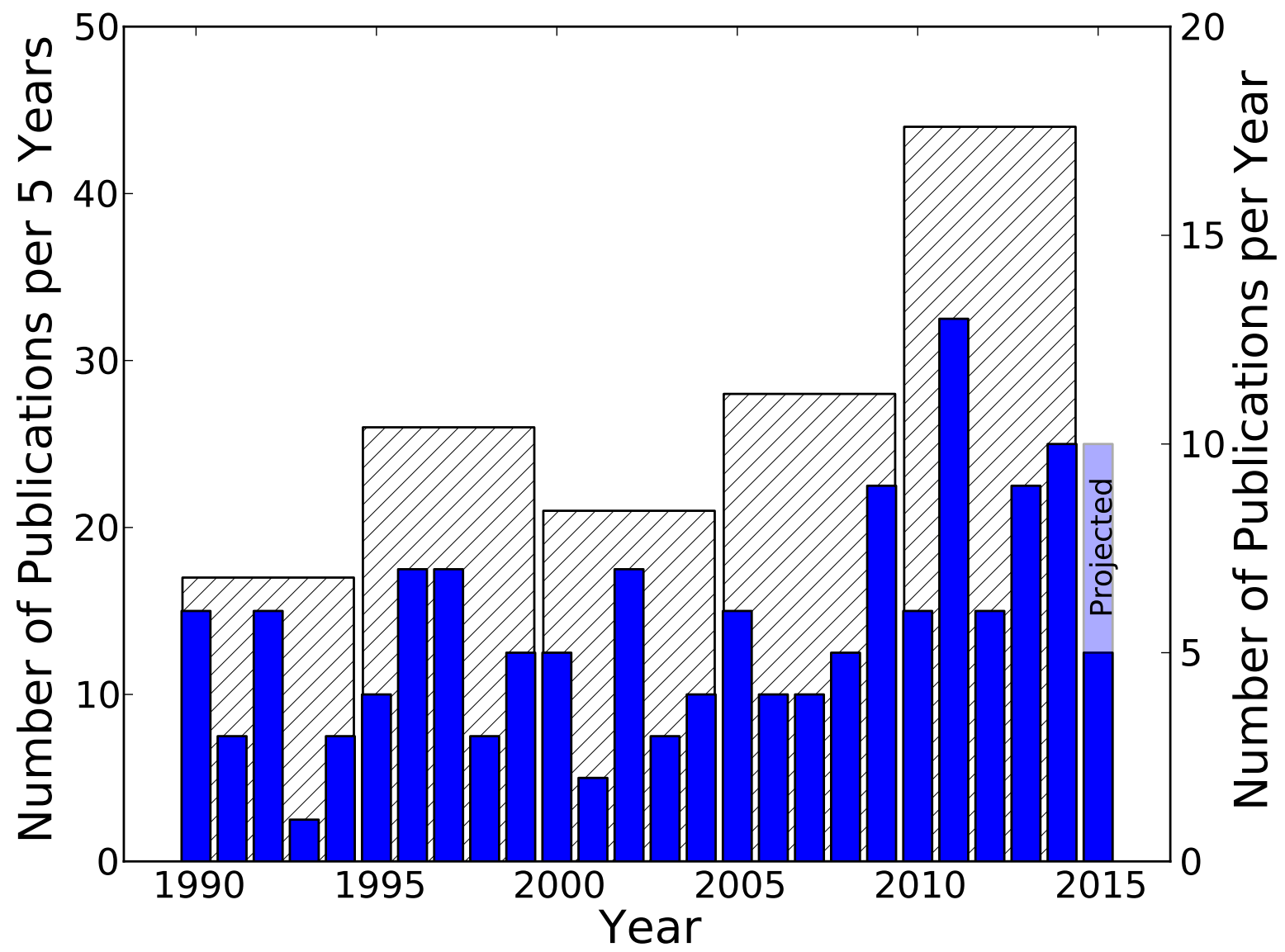

Figure 17: Number of publication per calendar year providing the data in Table 1. Five year bins of the count has indicated by the shaded bars.

photoionisation scheme [298]. By directly transferring the stopped beam to the PIPS detector, the alpha particles following the decay of ${ }^{254} \mathrm{No}$ could be counted and used to determine a production rate of $\sim 14$ $\mathrm{s}^{-1}$. Based on the level predictions, a first search for the $7 s 7 p^{1} \mathrm{P}_{1}$ excited state was performed however no clear resonance was found. A number of possible signatures were seen which when combined with more recent MCDF calculations and a doubling of the number of scanning lasers, will remain potential candidates for future experiments.

\section{Outlook}

\subsection{Recent output}

Figure 17 shows the number of publications (contributing to the data in Table 1) per calendar year, and with these binned in 5 yearly intervals, since 1990. At face value the last five years of research in the field would appear to have been highly productive. While it is tempting to associate this with a number of experiments and techniques coming to fruition, and indeed many have in the reviewed period, a number of single experiments give rise to multiple publications (for which no correction is made). That notwithstanding the field appears vibrant and productive. 


\subsection{Future prospects and challenges}

\subsubsection{Sensitivity and universality}

Great effort over the years has been applied to accessing weakly produced isotopes and isomers in the majority of chemical elements. With respect to the remaining challenges, late d-shell species, $\mathrm{Fe}-\mathrm{Ni}$, $\mathrm{Tc}-\mathrm{Pd}$ and $\mathrm{W}-\mathrm{Os}$, the majority of non-metals (excluding the noble gases) and elements beyond $Z=100$ lack reports of measurements in radioactive systems.

The majority of the late d-shell elements have had spectroscopy demonstrated on stable isotopes or have been produced in laser ion sources without spectroscopy being attempted. As such they are accessible and new spectroscopic results are realistically expected within the next few years. Once these isotopes are explored laser spectroscopy will have been applied to all elements bar species in the actinides (Section 7) and, at lower $Z$, a triangular region of the periodic table bordered on the perpendicular by the noble gases and the elements ${ }_{13} \mathrm{Al}-{ }_{32} \mathrm{Ge}-{ }_{51} \mathrm{Sb}-{ }_{83} \mathrm{Bi}$ on the diagonal - the "non-metals". The elements on the boundaries are themselves accessible (with the exception of frequency challenges in the element $\mathrm{Ge}$ ) but species within the region remain beyond the reach of present high sensitivity optical techniques (bulk samples of non-metals may be optically explored in high-lying atomic states [399] or exploiting techniques such as saturation spectroscopy [400]). Extraction of precision nuclear data remains however elusive. The prospects for extracting nuclear data in this region is returned to at the end of the section.

For the majority of the studied elements high sensitivity and high efficiency spectroscopy exists at least to secondary production levels of $10-100$ ions $\mathrm{s}^{-1}$. New experimental efforts focus on driving studies to nuclei produced at weaker levels or to those produced more abundantly but with accompanying high levels of isobaric (or other) contamination. Equally intense efforts are also being applied to primary production capabilities and new facilities are planned or being commissioned (below). The highest sensitivity spectroscopies are at present those involving resonance ionisation coupled to decay detection [183, 184, 244] and those involving spectroscopy of trapped species (Section 3.6) (assuming efficient loading of the device). The highest precisions are obtained in microwave or radiofrequency measurements of hyperfine intervals once shielding (and other external) perturbations are accounted for or removed. The accuracy and precision achieved has permitted extraction of nuclear data in the lightest elements (Section 5.1.2) and is readily sufficient for the study of heavier systems. Precision nuclear data extraction for the heavier systems will however only be possible with knowledge of the atomic field and mass shift factors.

\subsubsection{Extraction of nuclear parameters}

Valuable nuclear structural information, and a critical test of precision, can be made with isotopic charge radii data by attempting to extract isotonic trends from the data (in combination with data from other sources). The extraction closely resembles the evaluation of proton separation energies from mass measurements which (for separation energies) is straightforward and commonly attempted when interpreting new data. A comparable analysis with charge radii would be possible if either absolute $\left\langle r^{2}\right\rangle$ and precise atomic factors or $\delta\left\langle r^{2}\right\rangle^{Z, Z^{\prime}}$ were known (as highlighted in Section 6.1.4). Evaluation of isotonic changes in charge radii have been attempted with existing data and are presented in reference $[205,375]$.

The precision with which $\delta\left\langle r^{2}\right\rangle^{Z, Z^{\prime}}$ can be evaluated for isotopes away from the valley of stability critically depends on the accuracy of atomic F and M factors. Some confidence in the global evaluations of atomic $F$ and $M$ factors can be taken from the systematics observed in charge radii when viewed across the medium (and heavy) mass nuclei. In these regions no chain appears to display a significantly different local slope to its neighbour and no clearly rogue or suspect evaluations can be identified. To date however the accuracy required in non-optical measurements and in the evaluation of atomic 
parameters has not reached the precision required to provide isotonic trends away from the valley of stability [375]. Nearer to stability and especially for the light, low $Z$, isotopes useful isotonic charge radii trends can be extracted and examined relative to other nuclear observables [205].

At least two future non-optical experiments, the SCRIT facility [319] at RIKEN and the use of dielectronic recombination spectroscopy in storage rings [401] at GSI, aim to provide absolute $\left\langle r^{2}\right\rangle$ or precision $\delta\left\langle r^{2}\right\rangle$. At the SCRIT facility electron scattering measurements will be made on confined radioactive ions (with a high intensity electron beam providing both the confinement and production mechanisms). At the GSI ESR storage ring detection of recombined ions, following an inverse Auger process, already provides a spectroscopy akin to precision X-ray spectroscopy - measuring at meVprecision atomic lines in highly-charged ions [401].

An optical spectroscopic path to precision measurements of $\delta\left\langle r^{2}\right\rangle$ may however open at the future FAIR facility. The spectroscopy trap, SpecTrap, part of the HITRAP (highly charged ion trap) facility at FAIR [269], will provide an opportunity for spectroscopy of highly charged ions. A number of hydrogenic medium- $Z$ elements have $2 S_{1 / 2}-2 P_{1 / 2}$ and $2 S_{1 / 2}-2 P_{3 / 2}$ transitions within reach of high power tunable lasers (for example, $\mathrm{Si}^{13+}, \mathrm{P}^{14+}$ ). Similarly a sizeable number of lower charge state, higher $Z$, alkali-like elements also have these transitions in the (near) visible. Direct spectroscopy of these HCI, in the manner highlighted in the discussion of the Proton Puzzle, can provide extremely accurate measures of $\delta\left\langle r^{2}\right\rangle$ with precisions far greater than those achieved in valence transitions.

Results for radioactive isotopes from either experiment would act as calibration for optical data, either providing accurate absolute $\left\langle r^{2}\right\rangle$ or precision $\delta\left\langle r^{2}\right\rangle$ for stable and longer-lived (many seconds) radioactive isotopes. A series of such measurements along two isotone chains covering extremes of isotope shift measurements in isotopic chains, for example $N=50$ and $N=60$, would act to precisely calibrate more than 100 charge radii. Prior to such a global calibration, merely a few precise measurements of absolute $\left\langle r^{2}\right\rangle$ (or changes) could facilitate an exploration of the true confidence that should be assigned to the large scale Dirac-Fock calculations described in Section 2.3. At present the precision of these may be explored usefully in the relative comparisons of various atomic and ionic transitions. Resolving whether they are more accurate than their assigned confidence $(\sim 15 \%$ [199]) requires independent knowledge of (changes in) nuclear charge radii.

\subsubsection{Future experiments and facilities}

At all new facilities, and now the majority of the existing, laser spectroscopic studies takes place in experimental areas in which precision mass measurements, using on-line Penning traps or time-offlight techniques, are made [402]. Many facilities share common beam preparation sections and many targeted nuclear structure investigations are common to both. The combination of results of optical measurements and mass measurements is now a common feature in modern explorations. When both are achieved, especially powerful if simultaneously performed, it is possible to identify spins and structures and to assign excitation energies in mixed ground and (multi-)isomeric systems. In light species the twin approach further provides the precision nuclear mass required for extraction of velocity-shifted frequencies [28]. For all masses, the comparison of neutron separation energies and changes in nuclear charge radii provides an opportunity to compare the behaviour of the matter within a nucleus to that of the charge. Often, for example in the region around ${ }_{40} \mathrm{Zr}$, by virtue of the attractive proton-neutron interaction, a consistent and strongly systematic relative behaviour can be appreciated between the observables [82]. With their (presently) higher spectroscopic sensitivity and chemical insensitivity, mass measurements can critically act as a guide for targeting optical studies to newly detected regions of nuclear structural change.

On-line mass measurements with electrostatic multi-reflection time of flight spectrometers (MR-ToF) have been recently and powerfully realised at a number of facilities, notably ISOLDE [403]. The devices and other electrostatic traps, such as the ConeTrap [270, 271], promise future spectroscopic possibilities 
as they are readily suitable for laser spectroscopy. Spectroscopy of trapped ions (and atoms) features significantly in the reviewed work, a trend expected to continue with increasing experimental demands on sensitivity and precision.

As can be seen from Figure 1 there are still large areas of the nuclear chart yet to be studied. In order to expand into these regions the aforementioned evolution in equipment and techniques is required. This is in addition to the advances in the capabilities of radioactive beam facilities. Planned and ongoing upgrades to many of the existing facilities such as ISOLDE, JYFL and TRIUMF will enable many of those elements already studied to be pushed out towards both the proton and neutron drip lines. Exploring the nuclear landscape towards regions of ever increasing distance from the valley of stability will no doubt both test and stimulate further theoretical studies.

The longer term sees new, more powerful facilities coming on-line with the promise of more intense beams as well as those that have been hitherto chemically difficult to produce. Already collaborations at Fair, Spiral2, Frib and Triumf are looking into how best to utilise the unique capabilities that each of these next generation of radioactive beam facilities brings to the field.

\section{Conclusions}

Results from optical studies of radioactive nuclei were reviewed in 1979, by Jacquinot \& Klapisch [1], some 35 years ago. Since that time the field and associated nuclear structure results have been regularly or topically updated and reviewed [2-5]. At present, following 40 years of development, a wealth of techniques are in regular use worldwide and all stand ready to be exploited at the next generation of facilities (notably the FAIR, FRIB and SPIRAL2 facilities are all envisaged to include both low-energy areas and dedicated laser spectroscopy stations). Over time the field has matured to the point where laser-assisted production is routinely employed [214] and to one in which nuclear structural interest alone drives the spectroscopy (as opposed to experiments attempting access to previously inaccessible systems). Today targeted investigations address individual regions or nuclei as theoretical priorities demand and only a minority of chemical elements lie beyond the reach of on-line laser spectroscopy.

Laser spectroscopy continues to be a powerful and popular tool for the study of ground state nuclear properties, spanning the chart of the nuclides from the lightest to the heaviest systems. Over the past few years the development and application of beam bunchers and coolers as well as both in-source and collinear resonance ionisation techniques and trapping of ultra-cold atoms and ions has opened up new possibilities for the study of nuclei with high sensitivity and production rates of only a few atoms per second. The continual evolution of traditional laser spectroscopic methods ensures that despite having been pursued for over 30 years this field of study will be both active and fruitful for the foreseeable future.

\section{Acknowledgements}

The authors firstly wish to thank A. Voss for her excellent support throughout the process of this review, both in terms of organising references and for providing the basis for several of the figures. Our effort was made far easier with her tireless contributions. We also wish to thank K. Marinova for providing access to all data used in her updated table of nuclear ground state charge radi, A. Barzakh for providing unpublished Bi data and for all those who kindly provided figures.

This work has been supported by the Academy of Finland under the Finnish Centre of Excellence Program 2012-2017 (Nuclear and Accelerator Based Physics Program at JYFL) and TRIUmF which receives funding from the National Research Council of Canada (NRC). 


\section{References}

[1] P. Jacquinot and R. Klapisch, Reports on Progress in Physics 42, 773 (1979), URL http:// stacks . iop.org/0034-4885/42/i=5/a=001.

[2] E. W. Otten, in Treatise on Heavy Ion Science, edited by D. A. Bromley (Springer US, 1989), pp. 517-638, ISBN 978-1-4612-8045-3, URL http://dx.doi.org/10.1007/978-1-4613-0713-6_7.

[3] J. Billowes and P. Campbell, Journal of Physics G 21, 707 (1995).

[4] Kluge, H.-J and Nörtershäuser, Spectrochimica Acta 58B, 1031 (2003).

[5] B. Cheal and K. T. Flanagan, Journal of Physics G: Nuclear and Particle Physics 37, 113101 (2010), URL http://stacks.iop.org/0954-3899/37/i=11/a=113101.

[6] R. Neugart and G. Neyens, in The Euroschool Lectures on Physics with Exotic Beams, Vol. II, edited by J. Al-Khalili and E. Roeckl (Springer Berlin Heidelberg, 2006), vol. 700 of Lecture Notes in Physics, pp. 135-189, ISBN 978-3-540-33786-7, URL http://dx.doi.org/10.1007/ 3-540-33787-3_4.

[7] N. Stone, Nuclear Data Services, International Atomic Energy Agency (2014).

[8] J. Persson, Atomic Data and Nuclear Data Tables 99, 62 (2013), ISSN 0092-640X, URL http: //www.sciencedirect.com/science/article/pii/S0092640X1200085X.

[9] K. Blaum, J. Dilling, and W. Nörtershäuser, Physica Scripta 2013, 014017 (2013), URL http: //stacks.iop.org/1402-4896/2013/i=T152/a=014017.

[10] A. Kramida, Atomic Data and Nuclear Data Tables 96, 586 (2010), ISSN 0092-640X, URL http://www.sciencedirect.com/science/article/pii/S0092640X10000458.

[11] P. Cancio Pastor, L. Consolino, G. Giusfredi, P. De Natale, M. Inguscio, V. A. Yerokhin, and K. Pachucki, Phys. Rev. Lett. 108, 143001 (2012), URL http://link.aps.org/doi/10.1103/ PhysRevLett.108.143001.

[12] F. Marin, F. Minardi, F. S. Pavone, M. Inguscio, and G. W. F. Drake, Zeitschrift für Physik D Atoms, Molecules and Clusters 32, 285 (1995), ISSN 0178-7683, URL http://dx.doi.org/10. 1007/BF01437272.

[13] L. Wang, P. Mueller, K. Bailey, G. Drake, J. Greene, D. Henderson, R. Holt, R. Janssens, C. Jiang, Z. Lu, et al., PHYSICAL REVIEW LETTERS 93 (2004), ISSN 0031-9007.

[14] P. Mueller, I. A. Sulai, A. C. C. Villari, J. A. Alcántara-Núñez, R. Alves-Condé, K. Bailey, G. W. F. Drake, M. Dubois, C. Eléon, G. Gaubert, et al., Phys. Rev. Lett. 99, 252501 (2007), URL http://link.aps.org/doi/10.1103/PhysRevLett.99.252501.

[15] W. Nörtershäuser, R. Sánchez, G. Ewald, A. Dax, J. Behr, P. Bricault, B. A. Bushaw, J. Dilling, M. Dombsky, G. W. F. Drake, et al., Phys. Rev. A 83, 012516 (2011), URL http://link.aps . org/doi/10.1103/PhysRevA.83.012516.

[16] W. Nörtershäuser, T. Neff, R. Sánchez, and I. Sick, Phys. Rev. C 84, 024307 (2011), URL http://link.aps.org/doi/10.1103/PhysRevC.84.024307. 
[17] G. Ewald, W. Nörtershäuser, A. Dax, S. Götte, R. Kirchner, H.-J. Kluge, T. Kühl, R. Sanchez, A. Wojtaszek, B. A. Bushaw, et al., Phys. Rev. Lett. 93, 113002 (2004), URL http://link.aps . org/doi/10.1103/PhysRevLett.93.113002.

[18] G. Ewald, W. Nörtershäuser, A. Dax, S. Götte, R. Kirchner, H.-J. Kluge, T. Kühl, R. Sanchez, A. Wojtaszek, B. A. Bushaw, et al., Phys. Rev. Lett. 94, 039901 (2005), URL http://link. aps . org/doi/10.1103/PhysRevLett.94.039901.

[19] R. Sánchez, W. Nörtershäuser, G. Ewald, D. Albers, J. Behr, P. Bricault, B. A. Bushaw, A. Dax, J. Dilling, M. Dombsky, et al., Physical Review Letters 96, 033002 (2006).

[20] A. Krieger, K. Blaum, M. L. Bissell, N. Frömmgen, C. Geppert, M. Hammen, K. Kreim, M. Kowalska, J. Krämer, T. Neff, et al., Phys. Rev. Lett. 108, 142501 (2012), URL http: //link.aps.org/doi/10.1103/PhysRevLett.108.142501.

[21] A. Takamine, M. Wada, K. Okada, T. Sonoda, P. Schury, T. Nakamura, Y. Kanai, T. Kubo, I. Katayama, S. Ohtani, et al., Phys. Rev. Lett. 112, 162502 (2014), URL http://link.aps. org/doi/10.1103/PhysRevLett.112.162502.

[22] W. Nörtershäuser, D. Tiedemann, M. Žáková, Z. Andjelkovic, K. Blaum, M. Bissell, R. Cazan, G. Drake, C. Geppert, M. Kowalska, et al., Physical Review Letters 102, 062503 (2009).

[23] K. Okada, M. Wada, T. Nakamura, A. Takamine, V. Lioubimov, P. Schury, Y. Ishida, T. Sonoda, M. Ogawa, Y. Yamazaki, et al., Phys. Rev. Lett. 101, 212502 (2008), URL http://link.aps . org/doi/10.1103/PhysRevLett.101.212502.

[24] A. Takamine, M. Wada, K. Okada, T. Nakamura, P. Schury, T. Sonoda, V. Lioubimov, H. Iimura, Y. Yamazaki, Y. Kanai, et al., The European Physical Journal A 42, 369 (2009), ISSN 1434-6001, URL http://dx.doi.org/10.1140/epja/i2009-10883-5.

[25] M. Žáková, Z. Andjelkovic, M. L. Bissell, K. Blaum, G. W. F. Drake, C. Geppert, M. Kowalska, J. Krämer, A. Krieger, M. Lochmann, et al., Journal of Physics G: Nuclear and Particle Physics 37, 055107 (2010).

[26] K. Marinova, W. Geithner, M. Kowalska, K. Blaum, S. Kappertz, M. Keim, S. Kloos, G. Kotrotsios, P. Lievens, R. Neugart, et al., Physical Review C 84, 034313 (2011).

[27] W. Geithner, B. A. Brown, K. M. Hilligsœe, S. Kappertz, M. Keim, G. Kotrotsios, P. Lievens, K. Marinova, R. Neugart, H. Simon, et al., Phys. Rev. C 71, 064319 (2005), URL http://link. aps.org/doi/10.1103/PhysRevC.71.064319.

[28] W. Geithner, T. Neff, G. Audi, K. Blaum, P. Delahaye, H. Feldmeier, S. George, C. Guénaut, F. Herfurth, A. Herlert, et al., Phys. Rev. Lett. 101, 252502 (2008), URL http://link.aps . org/doi/10.1103/PhysRevLett.101.252502.

[29] Y. P. Gangrsky, D. V. Karaivanov, K. P. Marinova, B. N. Markov, L. M. Melnikova, G. V. Mishinsky, S. G. Zemlyanoi, and V. I. Zhemenik, The European Physical Journal A - Hadrons and Nuclei 3, 313 (1998), ISSN 1434-6001, URL http://dx.doi.org/10.1007/s100500050185.

[30] D. T. Yordanov, M. L. Bissell, K. Blaum, M. De Rydt, C. Geppert, M. Kowalska, J. Krämer, K. Kreim, A. Krieger, P. Lievens, et al., Phys. Rev. Lett. 108, 042504 (2012), URL http://link. aps.org/doi/10.1103/PhysRevLett.108.042504. 
[31] J. Krämer, K. Blaum, M. D. Rydt, K. Flanagan, C. Geppert, M. Kowalska, P. Lievens, R. Neugart, G. Neyens, W. Nörtershäuser, et al., Physics Letters B 678, 465 (2009), ISSN 0370-2693, URL http://www.sciencedirect.com/science/article/pii/S037026930900776X.

[32] T. G. Cooper, J. Billowes, P. Campbell, and M. R. Pearson, Journal of Physics G: Nuclear and Particle Physics 22, 99 (1996), URL http://stacks.iop.org/0954-3899/22/i=1/a=008.

[33] J. M. G. Levins, J. Billowes, P. Campbell, and M. R. Pearson, Journal of Physics G: Nuclear and Particle Physics 23, 1145 (1997), URL http://stacks.iop.org/0954-3899/23/i=9/a=015.

[34] K. Wendt, C. Mattolat, T. Gottwald, T. Kron, S. Raeder, S. Rothe, F. Schwellnus, and H. Tomita, Phys. Rev. A 88, 052510 (2013), URL http://link.aps.org/doi/10.1103/PhysRevA.88. 052510.

[35] A. Klein, B. A. Brown, U. Georg, M. Keim, P. Lievens, R. Neugart, M. Neuroth, R. E. Silverans, L. Vermeeren, and I. S. O. L. D. E. Collaboration, Nuclear Physics A 607, 1 (1996), ISSN 03759474, URL http://www.sciencedirect.com/science/article/pii/0375947496001923.

[36] K. Blaum, W. Geithner, J. Lassen, P. Lievens, K. Marinova, and R. Neugart, Nuclear Physics A 799, 30 (2008), ISSN 0375-9474, URL http://www.sciencedirect.com/science/article/ pii/S0375947407007841.

[37] A. Behrle, M. Koschorreck, and M. Köhl, Phys. Rev. A 83, 052507 (2011), URL http://link. aps.org/doi/10.1103/PhysRevA.83.052507.

[38] J. Papuga, M. L. Bissell, K. Kreim, K. Blaum, B. A. Brown, M. De Rydt, R. F. Garcia Ruiz, H. Heylen, M. Kowalska, R. Neugart, et al., Phys. Rev. Lett. 110, 172503 (2013), URL http: //link.aps.org/doi/10.1103/PhysRevLett.110.172503.

[39] K. Kreim, M. L. Bissell, J. Papuga, K. Blaum, M. D. Rydt, R. F. G. Ruiz, S. Goriely, H. Heylen, M. Kowalska, R. Neugart, et al., Physics Letters B 731, 97 (2014), ISSN 0370-2693, URL http: //www.sciencedirect.com/science/article/pii/S0370269314001038.

[40] J. Papuga, M. L. Bissell, K. Kreim, C. Barbieri, K. Blaum, M. De Rydt, T. Duguet, R. F. Garcia Ruiz, H. Heylen, M. Kowalska, et al., Phys. Rev. C 90, 034321 (2014), URL http: //link.aps.org/doi/10.1103/PhysRevC.90.034321.

[41] K. Minamisono, B. Barquest, G. Bollen, K. Cooper, K. Hammerton, M. Hughes, P. Mantica, D. Morrissey, R. Ringle, J. Rodriguez, et al., Hyperfine Interactions 230, 57 (2015), ISSN 03043843, URL http://dx.doi.org/10.1007/s10751-014-1089-5.

[42] D. M. Rossi, K. Minamisono, H. B. Asberry, G. Bollen, B. A. Brown, K. Cooper, B. Isherwood, P. F. Mantica, A. Miller, D. J. Morrissey, et al., Phys. Rev. C 92, 014305 (2015), URL http: //link.aps.org/doi/10.1103/PhysRevC.92.014305.

[43] A. M. Martensson-Pendrill, L. Pendrill, A. Salomonson, A. Ynnerman, and H. Warston, Journal of Physics B: Atomic, Molecular and Optical Physics 23, 1749 (1990), URL http://stacks .iop. org $/ 0953-4075 / 23 / i=11 / a=012$.

[44] J. A. Behr, A. Gorelov, T. Swanson, O. Häusser, K. P. Jackson, M. Trinczek, U. Giesen, J. M. D'Auria, R. Hardy, T. Wilson, et al., Phys. Rev. Lett. 79, 375 (1997), URL http://link.aps. org/doi/10.1103/PhysRevLett.79.375. 
[45] R. F. G. Ruiz, M. L. Bissell, K. Blaum, N. Frömmgen, M. Hammen, J. D. Holt, M. Kowalska, K. Kreim, J. Menéndez, R. Neugart, et al., Phys. Rev. C 91, 041304 (2015), URL http://link. aps.org/doi/10.1103/PhysRevC.91.041304.

[46] L. Vermeeren, R. E. Silverans, P. Lievens, A. Klein, R. Neugart, C. Schulz, and F. Buchinger, Phys. Rev. Lett. 68, 1679 (1992), URL http://link.aps.org/doi/10.1103/PhysRevLett.68.1679.

[47] L. Vermeeren, P. Lievens, R. E. Silverans, U. Georg, M. Keim, A. Klein, R. Neugart, M. Neuroth, F. Buchinger, and t. I. Collaboration, Journal of Physics G: Nuclear and Particle Physics 22, 1517 (1996), URL http://stacks.iop.org/0954-3899/22/i=10/a=014.

[48] M. Avgoulea, Y. P. Gangrsky, K. P. Marinova, S. G. Zemlyanoi, S. Fritzsche, D. Iablonskyi, C. Barbieri, E. C. Simpson, P. D. Stevenson, J. Billowes, et al., Journal of Physics G: Nuclear and Particle Physics 38, 025104 (2011), URL http://stacks.iop.org/0954-3899/38/i=2/a= 025104.

[49] Y. P. Gangrsky, K. P. Marinova, and S. G. Zemlyanoi, Journal of Physics B: Atomic, Molecular and Optical Physics 28, 957 (1995), URL http://stacks.iop.org/0953-4075/28/i=6/a=011.

[50] Y. P. Gangrsky, K. P. Marinova, S. G. Zemlyanoi, I. D. Moore, J. Billowes, P. Campbell, K. T. Flanagan, D. H. Forest, J. A. R. Griffith, J. Huikari, et al., Journal of Physics G: Nuclear and Particle Physics 30, 1089 (2004), URL http://stacks.iop.org/0954-3899/30/i=9/a=009.

[51] P. Aufmuth, K. Heilig, and A. Steudel, Atomic Data and Nuclear Data Tables 37, 455 (1987), ISSN 0092-640X, URL http://www.sciencedirect.com/science/article/pii/0092640X87900283.

[52] A. Klose, K. Minamisono, and P. F. Mantica, Phys. Rev. A 88, 042701 (2013), URL http: //link.aps.org/doi/10.1103/PhysRevA.88.042701.

[53] F. C. Charlwood, J. Billowes, P. Campbell, B. Cheal, T. Eronen, D. H. Forest, S. Fritzsche, M. Honma, A. Jokinen, I. D. Moore, et al., Physics Letters B 690, 346 (2010), ISSN 0370-2693, URL http://www.sciencedirect.com/science/article/pii/S0370269310006520.

[54] D. M. Benton, E. C. A. Cochrane, and J. A. R. Griffith, Journal of Physics B: Atomic, Molecular and Optical Physics 30, 5359 (1997), URL http://stacks . iop.org/0953-4075/30/i=23/a=005.

[55] A. Steudel, U. Triebe, and D. Wendlandt, Zeitschrift für Physik A Atoms and Nuclei 296, 189 (1980), ISSN 0939-7922, URL http://dx.doi.org/10.1007/BF01415832.

[56] U. Köster, N. J. Stone, K. T. Flanagan, J. R. Stone, V. N. Fedosseev, K. L. Kratz, B. A. Marsh, T. Materna, L. Mathieu, P. L. Molkanov, et al., Phys. Rev. C 84, 034320 (2011), URL http: //link.aps.org/doi/10.1103/PhysRevC.84.034320.

[57] P. Vingerhoets, K. T. Flanagan, J. Billowes, M. L. Bissell, K. Blaum, B. Cheal, M. D. Rydt, D. H. Forest, C. Geppert, M. Honma, et al., Physics Letters B 703, 34 (2011), ISSN 0370-2693, URL http://www.sciencedirect.com/science/article/pii/S0370269311008549.

[58] L. Weissman, U. Köster, R. Catherall, S. Franchoo, U. Georg, O. Jonsson, V. N. Fedoseyev, V. I. Mishin, M. D. Seliverstov, J. Van Roosbroeck, et al. ((IS365 Collaboration and ISOLDE Collaboration)), Phys. Rev. C 65, 024315 (2002), URL http://link.aps.org/doi/10.1103/ PhysRevC.65.024315. 
[59] N. J. Stone, U. KÖster, J. R. Stone, D. V. Fedorov, V. N. Fedoseyev, K. T. Flanagan, M. Hass, and S. Lakshmi, Phys. Rev. C 77, 067302 (2008), URL http://link.aps.org/doi/10.1103/ PhysRevC.77.067302.

[60] T. E. Cocolios, A. N. Andreyev, B. Bastin, N. Bree, J. Büscher, J. Elseviers, J. Gentens, M. Huyse, Y. Kudryavtsev, D. Pauwels, et al., Phys. Rev. Lett. 103, 102501 (2009), URL http://link. aps.org/doi/10.1103/PhysRevLett.103.102501.

[61] K. T. Flanagan, P. Vingerhoets, M. Avgoulea, J. Billowes, M. L. Bissell, K. Blaum, B. Cheal, M. De Rydt, V. N. Fedosseev, D. H. Forest, et al., Phys. Rev. Lett. 103, 142501 (2009), URL http://link.aps.org/doi/10.1103/PhysRevLett.103.142501.

[62] T. E. Cocolios, A. N. Andreyev, B. Bastin, N. Bree, J. Büscher, J. Elseviers, J. Gentens, M. Huyse, Y. Kudryavtsev, D. Pauwels, et al., Phys. Rev. C 81, 014314 (2010), URL http://link.aps . org/doi/10.1103/PhysRevC.81.014314.

[63] P. Campbell, J. Billowes, and I. S. Grant, Journal of Physics B: Atomic, Molecular and Optical Physics 30, 2351 (1997), URL http://stacks.iop.org/0953-4075/30/i=10/a=010.

[64] B. Cheal, J. Billowes, M. L. Bissell, K. Blaum, F. C. Charlwood, K. T. Flanagan, D. H. Forest, S. Fritzsche, C. Geppert, A. Jokinen, et al., Phys. Rev. C 82, 051302R (2010), URL http: //link.aps.org/doi/10.1103/PhysRevC.82.051302.

[65] E. Mané, B. Cheal, J. Billowes, M. L. Bissell, K. Blaum, F. C. Charlwood, K. T. Flanagan, D. H. Forest, C. Geppert, M. Kowalska, et al., Phys. Rev. C 84, 024303 (2011), URL http: //link.aps.org/doi/10.1103/PhysRevC.84.024303.

[66] T. J. Procter, J. Billowes, M. L. Bissell, K. Blaum, F. C. Charlwood, B. Cheal, K. T. Flanagan, D. H. Forest, S. Fritzsche, C. Geppert, et al., Phys. Rev. C 86, 034329 (2012), URL http: //link.aps.org/doi/10.1103/PhysRevC.86.034329.

[67] B. Cheal, E. Mané, J. Billowes, M. L. Bissell, K. Blaum, B. A. Brown, F. C. Charlwood, K. T. Flanagan, D. H. Forest, C. Geppert, et al., Phys. Rev. Lett. 104, 252502 (2010), URL http: //link.aps.org/doi/10.1103/PhysRevLett.104.252502.

[68] M. Keim, E. Arnold, W. Borchers, U. Georg, A. Klein, R. Neugart, L. Vermeeren, R. E. Silverans, and P. Lievens, Nuclear Physics A 586, 219 (1995), ISSN 0375-9474, URL http: //www.sciencedirect.com/science/article/pii/037594749400786M.

[69] P. Lievens, E. Arnold, W. Borchers, U. Georg, M. Keim, A. Klein, R. Neugart, L. Vermeeren, and R. E. Silverans, Europhys. Lett. 33, 11 (1996).

[70] T. Kong, A. Gorelov, C. Höhr, T. Wiebe, A. Chatwin-Davies, A. Berman, S. Behling, D. Ashery, G. Gwinner, M. R. Pearson, et al., Journal of Physics B: Atomic, Molecular and Optical Physics 44, 215004 (2011), URL http://stacks.iop.org/0953-4075/44/i=21/a=215004.

[71] E. Mané, A. Voss, J. A. Behr, J. Billowes, T. Brunner, F. Buchinger, J. E. Crawford, J. Dilling, S. Ettenauer, C. D. P. Levy, et al., Phys. Rev. Lett. 107, 212502 (2011), URL http://Iink.aps . org/doi/10.1103/PhysRevLett.107.212502.

[72] T. Procter, J. Behr, J. Billowes, F. Buchinger, B. Cheal, J. Crawford, J. Dilling, A. Garnsworthy, A. Leary, C. Levy, et al., The European Physical Journal A 51, 23 (2015), ISSN 1434-6001, URL http://dx.doi.org/10.1140/epja/i2015-15023-2. 
[73] G. Shimkaveg, W. W. Quivers, R. R. Dasari, C. H. Holbrow, P. G. Pappas, M. A. Attili, J. E. Thomas, D. E. Murnick, and M. S. Feld, Phys. Rev. Lett. 53, 2230 (1984), URL http://link. aps.org/doi/10.1103/PhysRevLett.53.2230.

[74] X. Zhao, S. G. Crane, R. Guckert, and D. J. Vieira, Phys. Rev. A 60, 4730 (1999), URL http: //link.aps.org/doi/10.1103/PhysRevA.60.4730.

[75] F. Buchinger, E. B. Ramsay, E. Arnold, W. Neu, R. Neugart, K. Wendt, R. E. Silverans, P. Lievens, L. Vermeeren, D. Berdichevsky, et al., Phys. Rev. C 41, 2883 (1990), URL http://link.aps.org/doi/10.1103/PhysRevC.41.2883.

[76] B. Cheal, M. D. Gardner, M. Avgoulea, J. Billowes, M. L. Bissell, P. Campbell, T. Eronen, K. T. Flanagan, D. H. Forest, J. Huikari, et al., Physics Letters B 645, 133 (2007), ISSN 0370-2693, URL http://www.sciencedirect.com/science/article/pii/S037026930601608X.

[77] K. Baczynska, J. Billowes, P. Campbell, F. C. Charlwood, B. Cheal, T. Eronen, D. H. Forest, A. Jokinen, T. Kessler, I. D. Moore, et al., Journal of Physics G: Nuclear and Particle Physics 37, 105103 (2010), URL http://stacks.iop.org/0954-3899/37/i=10/a=105103.

[78] P. Campbell, H. L. Thayer, J. Billowes, P. Dendooven, K. T. Flanagan, D. H. Forest, J. A. R. Griffith, J. Huikari, A. Jokinen, R. Moore, et al., Phys. Rev. Lett. 89, 082501 (2002), URL http://link.aps.org/doi/10.1103/PhysRevLett.89.082501.

[79] D. H. Forest, J. Billowes, P. Campbell, P. Dendooven, K. T. Flanagan, J. A. R. Griffith, J. Huikari, A. Jokinen, R. Moore, A. Nieminen, et al., Journal of Physics G: Nuclear and Particle Physics 28, L63 (2002), URL http://stacks.iop.org/0954-3899/28/i=12/a=101.

[80] H. L. Thayer, J. Billowes, P. Campbell, P. Dendooven, K. T. Flanagan, D. H. Forest, J. A. R. Griffith, J. Huikari, A. Jokinen, R. Moore, et al., Journal of Physics G: Nuclear and Particle Physics 29, 2247 (2003), URL http://stacks.iop.org/0954-3899/29/i=9/a=318.

[81] B. Cheal, K. Baczynska, J. Billowes, P. Campbell, F. C. Charlwood, T. Eronen, D. H. Forest, A. Jokinen, T. Kessler, I. D. Moore, et al., Phys. Rev. Lett. 102, 222501 (2009), URL http: //link.aps.org/doi/10.1103/PhysRevLett.102.222501.

[82] F. C. Charlwood, K. Baczynska, J. Billowes, P. Campbell, B. Cheal, T. Eronen, D. H. Forest, A. Jokinen, T. Kessler, I. D. Moore, et al., Physics Letters B 674, 23 (2009), ISSN 0370-2693, URL http://www.sciencedirect.com/science/article/pii/S0370269309002470.

[83] D. H. Forest, R. A. Powis, E. C. A. Cochrane, J. A. R. Griffith, and G. Tungate, Journal of Physics G: Nuclear and Particle Physics 41, 025106 (2014), URL http://stacks.iop.org/0954-3899/ $41 / i=2 / a=025106$.

[84] A. Steudel, Zeitschrift für Physik 132, 429 (1952), ISSN 0044-3328, URL http://dx.doi.org/ 10.1007/BF01333189.

[85] P. E. G. Baird, Proceedings of the Royal Society of London. A. Mathematical and Physical Sciences 351, 267 (1976), http://rspa.royalsocietypublishing.org/content/351/1665/267. full.pdf+html, URL http://rspa.royalsocietypublishing.org/content/351/1665/267. abstract.

[86] R. Ferrer, N. Bree, T. E. Cocolios, I. G. Darby, H. D. Witte, W. Dexters, J. Diriken, J. Elseviers, S. Franchoo, M. Huyse, et al., Physics Letters B 728, 191 (2014), ISSN 0370-2693, URL http: //www.sciencedirect.com/science/article/pii/S0370269313009611. 
[87] U. Dinger, J. Eberz, G. Huber, R. Menges, R. Kirchner, O. Klepper, T. Ku“hl, and D. Marx, Nuclear Physics A 503, 331 (1989), ISSN 0375-9474, URL http://www.sciencedirect.com/ science/article/pii/037594748990239X.

[88] B. A. Marsh, Ph.D. thesis, The University of Manchester (2007).

[89] D. T. Yordanov, D. L. Balabanski, J. Bieroń, M. L. Bissell, K. Blaum, I. Budinčević, S. Fritzsche, N. Frömmgen, G. Georgiev, C. Geppert, et al., Phys. Rev. Lett. 110, 192501 (2013), URL http: //link.aps.org/doi/10.1103/PhysRevLett.110.192501.

[90] F. Le Blanc et al., Eur. Phys. J. A 15, 49 (2002), ISSN 1434-6001.

[91] F. L. Blanc, L. Cabaret, E. Cottereau, J. E. Crawford, S. Essabaa, J. Genevey, R. Horn, G. Huber, J. Lassen, J. K. P. Lee, et al., Phys. Rev. C 72, 034305 (2005), URL http://link.aps.org/doi/ 10.1103/PhysRevC.72.034305.

[92] H. G. Kuhn and R. Turner, Proceedings of the Royal Society of London. Series A. Mathematical and Physical Sciences 265, 39 (1961), http://rspa.royalsocietypublishing.org/content/ 265/1320/39.full.pdf+html, URL http://rspa.royalsocietypublishing.org/content/ 265/1320/39. abstract.

[93] R. Lecordier, Physics Letters A 72, 327 (1979), ISSN 0375-9601, URL http://www . sciencedirect.com/science/article/pii/0375960179904845.

[94] R. Sifi, F. Blanc, N. Barré, L. Cabaret, J. Crawford, M. Ducourtieux, S. Essabaa, J. Genevey, G. Huber, M. Kowalska, et al., Hyperfine Interactions 171, 173 (2006), ISSN 0304-3843, URL http://dx.doi.org/10.1007/s10751-006-9505-0.

[95] W. Borchers, E. Arnold, W. Neu, R. Neugart, K. Wendt, and G. Ulm, Physics Letters B 216, 7 (1989), ISSN 0370-2693, URL http://www.sciencedirect.com/science/article/pii/ 0370269389913592.

[96] M. P. Dancy, M. A. Skelton, J. Billowes, D. A. Eastham, D. E. Evans, I. S. Grant, J. A. R. Griffith, P. M. Walker, D. D. Warner, and S. A. Wells, Journal of Physics G: Nuclear and Particle Physics 18, L67 (1992), URL http://stacks.iop.org/0954-3899/18/i=3/a=002.

[97] R. Moore, A. M. Bruce, P. Dendooven, J. Billowes, P. Campbell, A. Ezwam, K. T. Flanagan, D. H. Forest, J. Huikari, A. Jokinen, et al., Physics Letters B 547, 200 (2002), ISSN 0370-2693, URL http://www.sciencedirect.com/science/article/pii/S0370269302027843.

[98] H. Iimura, M. Koizumi, M. Miyabe, M. Oba, T. Shibata, N. Shinohara, Y. Ishida, T. Horiguchi, and H. Schuessler, Physical Review C 68, 054328 (2003).

[99] T. E. Cocolios, Master's thesis, McGill University (2005).

[100] Y. Ishida, H. Iimura, S. Ichikawa, and T. Horiguchi, Phys. Rev. C 59, 1794 (1999), URL http: //link.aps.org/doi/10.1103/PhysRevC.59.1794.

[101] B. Cheal, M. Avgoulea, J. Billowes, P. Campbell, K. T. Flanagan, D. H. Forest, M. D. Gardner, J. Huikari, B. A. Marsh, A. Nieminen, et al., Journal of Physics G: Nuclear and Particle Physics 29, 2479 (2003), URL http://stacks.iop.org/0954-3899/29/i=11/a=003.

[102] H. Iimura et al., Ann. Rep. Jaeri Tandem \& V. D.G 12 JAERI-Review 94-008, 1993. 
[103] V. S. Letokhov, V. I. Mishin, S. K. Sekatsky, V. N. Fedoseyev, G. D. Alkhazov, A. E. Barzakh, V. P. Denisov, and V. E. Starodubsky, Journal of Physics G: Nuclear and Particle Physics 18, 1177 (1992), URL http://stacks .iop.org/0954-3899/18/i=7/a=008.

[104] M. Hong-Liang, L. Mao-Sheng, and Y. Fu-Jia, Chinese Physics Letters 18, 903 (2001), URL http://stacks.iop.org/0256-307X/18/i=7/a=319.

[105] G. D. Alkhazov, A. E. Barzakh, H. Huhnermann, K. Kesper, A. Mazumdar, W. Moller, R. Otto, V. N. Pantelejev, A. G. Poljakov, C. Reese, et al., Journal of Physics B: Atomic, Molecular and Optical Physics 25, 571 (1992), URL http://stacks.iop.org/0953-4075/25/i=2/a=023.

[106] J. G. England, I. S. Grant, J. A. R. Griffith, D. E. Evans, D. A. Eastham, G. W. A. Newton, and P. M. Walker, Journal of Physics G: Nuclear and Particle Physics 16, 105 (1990), URL http://stacks.iop.org/0954-3899/16/i=1/a=014.

[107] G. D. Alkhazov, A. E. Barzakh, V. A. Bolshakov, V. P. Denisov, V. S. Ivanov, Y. Y. Sergeyev, I. Y. Chubukov, V. I. Tikhonov, V. S. Letokhov, V. I. Mishin, et al., Zeitschrift für Physik A Atomic Nuclei 337, 257 (1990), ISSN 0939-7922, URL http://dx.doi.org/10.1007/BF01289691.

[108] O. Becker, K. Enders, G. Werth, and J. Dembczynski, Phys. Rev. A 48, 3546 (1993), URL http://link.aps.org/doi/10.1103/PhysRevA.48.3546.

[109] K. Enders, E. Stachowska, G. Marx, C. Zölch, U. Georg, J. Dembczynski, and G. Werth (ISOLDE Collaboration), Phys. Rev. A 56, 265 (1997), URL http://link.aps.org/doi/10. 1103/PhysRevA.56.265.

[110] A. E. Barzakh, D. V. Fedorov, A. M. Ionan, V. S. Ivanov, F. V. Moroz, K. A. Mezilev, S. Y. Orlov, V. N. Panteleev, and Y. Volkov, The European Physical Journal A - Hadrons and Nuclei 22, 69 (2004), ISSN 1434-6001, URL http://dx.doi.org/10.1140/epja/i2003-10231-y.

[111] S. K. Borisov et al., Sov. Phys. JETP 66, 885 (1987).

[112] G. D. Alkhazov, A. E. Barzakh, V. P. Denisov, V. S. Ivanov, I. Y. Chubukov, N. B. Buyanov, V. S. Letokhov, V. I. Mishin, S. K. Sekatskii, and V. N. Fedoseev, JETP Letters 48, 413 (1988).

[113] G. D. Alkhasov et al., Isotopic changes of gadolinium nuclei charge radii and magic nucleus charge radius ${ }^{146}$ Gd (in russian), Akad. Nauk USSR, Leningrad Inst. Yad. Fis., preprint No. 1417 (1988).

[114] A. E. Barzakh, D. V. Fedorov, A. M. Ionan, V. S. Ivanov, F. V. Moroz, K. A. Mezilev, S. Y. Orlov, V. N. Panteleev, and Y. M. Volkov, Phys. Rev. C 72, 017301 (2005), URL http://link. aps.org/doi/10.1103/PhysRevC.72.017301.

[115] G. D. Alkhazov, A. E. Barzakh, V. P. Denisov, V. S. Ivanov, I. Y. Chubukov, V. S. Letokhov, V. I. Mishin, S. K. Sekatsky, and V. N. Fedoseyev, Zeitschrift für Physik A Atomic Nuclei 337, 367 (1990), ISSN 0939-7922, URL http://dx.doi.org/10.1007/BF01294972.

[116] K. Heilig, Hyperfine Interactions 24, 349 (1985), ISSN 0304-3843, URL http://dx.doi .org/10. $1007 / \mathrm{BF} 02354820$.

[117] A. E. Barzakh, I. Y. Chubukov, D. V. Fedorov, V. N. Panteleev, M. D. Seliverstov, and Y. M. Volkov, Phys. Rev. C 61, 034304 (2000), URL http://link.aps.org/doi/10.1103/PhysRevC. 61.034304. 
[118] G. D. Sprouse, J. Das, T. Lauritsen, J. Schecker, A. Berger, J. Billowes, C. H. Holbrow, H. E. Mahnke, and S. L. Rolston, Phys. Rev. Lett. 63, 1463 (1989), URL http://link.aps.org/doi/ 10.1103/PhysRevLett.63.1463.

[119] C. Schulz, E. Arnold, W. Borchers, W. Neu, R. Neugart, M. Neuroth, E. W. Otten, M. Scherf, K. Wendt, P. Lievens, et al., Journal of Physics B: Atomic, Molecular and Optical Physics 24, 4831 (1991), URL http://stacks.iop.org/0953-4075/24/i=22/a=020.

[120] M. L. Bissell, K. T. Flanagan, M. D. Gardner, M. Avgoulea, J. Billowes, P. Campbell, B. Cheal, T. Eronen, D. H. Forest, J. Huikari, et al., Physics Letters B 645, 330 (2007), ISSN 0370-2693, URL http://www.sciencedirect.com/science/article/pii/S037026930601625X.

[121] K. Pandey, A. K. Singh, P. V. K. Kumar, M. V. Suryanarayana, and V. Natarajan, Phys. Rev. A 80, 022518 (2009), URL http://link.aps.org/doi/10.1103/PhysRevA.80.022518.

[122] U. Georg, W. Borchers, M. Keim, A. Klein, P. Lievens, R. Neugart, M. Neuroth, P. M. Rao, and C. Schulz, The European Physical Journal A - Hadrons and Nuclei 3, 225 (1998), ISSN 1434-6001, URL http://dx.doi.org/10.1007/s100500050172.

[123] A. Anastassov, Y. P. Gangrsky, B. K. Kul'djanov, K. P. Marinova, B. N. Markov, and S. G. Zemlyanoi, Zeitschrift für Physik A Hadrons and Nuclei 348, 177 (1994), ISSN 0939-7922, URL http://dx.doi.org/10.1007/BF01291915.

[124] N. Boos, F. Le Blanc, M. Krieg, J. Pinard, G. Huber, M. D. Lunney, D. Le Du, R. Meunier, M. Hussonnois, O. Constantinescu, et al., Phys. Rev. Lett. 72, 2689 (1994), URL http://link. aps.org/doi/10.1103/PhysRevLett.72.2689.

[125] J. M. G. Levins, D. M. Benton, J. Billowes, P. Campbell, T. G. Cooper, P. Dendooven, D. E. Evans, D. H. Forest, I. S. Grant, J. A. R. Griffith, et al., Phys. Rev. Lett. 82, 2476 (1999), URL http://link.aps.org/doi/10.1103/PhysRevLett.82.2476.

[126] G. Yeandle, J. Billowes, P. Campbell, E. C. A. Cochrane, P. Dendooven, D. E. Evans, J. A. R. Griffith, J. Huikari, A. Jokinen, I. D. Moore, et al., Journal of Physics G: Nuclear and Particle Physics 26, 839 (2000), URL http://stacks.iop.org/0954-3899/26/i=6/a=307.

[127] A. Nieminen, P. Campbell, J. Billowes, D. H. Forest, J. A. R. Griffith, J. Huikari, A. Jokinen, I. D. Moore, R. Moore, G. Tungate, et al., Physical Review Letters 88, 094801 (2002).

[128] M. Wakasugi, W. G. Jin, M. G. Hies, T. T. Inamura, T. Murayama, T. Ariga, T. Ishizuka, T. Wakui, H. Katsuragawa, J. Z. Ruan, et al., Phys. Rev. C 53, 611 (1996), URL http://link. aps.org/doi/10.1103/PhysRevC.53.611.

[129] M. L. Bissell, K. Baczynska, J. Billowes, P. Campbell, B. Cheal, T. Eronen, D. H. Forest, M. D. Gardner, I. D. Moore, B. Tordoff, et al., Phys. Rev. C 74, 047301 (2006), URL http://link. aps.org/doi/10.1103/PhysRevC.74.047301.

[130] J. Lee, J. Chen, and A. E. Leanhardt, Journal of Physics B: Atomic, Molecular and Optical Physics 46, 075003 (2013), URL http://stacks.iop.org/0953-4075/46/i=7/a=075003.

[131] K. H. Bürger, B. Burghardt, S. Büttgenbach, R. Harzer, H. Hoeffgen, G. Meisel, and F. Träber, Zeitschrift für Physik A Atoms and Nuclei 307, 201 (1982), ISSN 0939-7922, URL http://dx. doi.org/10.1007/BF01438641. 
[132] J. R. Kropp, H. D. Kronfeldt, A. Lucas, and R. Winkler, Physica B + C 138, 335 (1986), ISSN 0378-4363, URL http://www.sciencedirect.com/science/article/pii/0378436386900161.

[133] A. P. Hines and J. S. Ross, Phys. Rev. 126, 2105 (1962), URL http://link.aps.org/doi/10. 1103/PhysRev. 126.2105.

[134] G. Nöldeke, A. Steudel, K. E. Wallach, and H. Walther, Zeitschrift für Physik 170, 22 (1962), ISSN 0044-3328, URL http://dx.doi.org/10.1007/BF01378306.

[135] P. Aufmuth and E. Wöbker, Zeitschrift für Physik A Atoms and Nuclei 321, 65 (1985), ISSN 0939-7922, URL http://dx.doi.org/10.1007/BF01411946.

[136] D. Verney, L. Cabaret, J. E. Crawford, H. T. Duong, B. Fricke, J. Genevey, G. Huber, F. Ibrahim, M. Krieg, F. Le Blanc, et al., The European Physical Journal A - Hadrons and Nuclei 30, 489 (2006), ISSN 1434-6001, URL http://dx.doi.org/10.1140/epja/i2006-10140-7.

[137] T. Hilberath, S. Becker, G. Bollen, H. J. Kluge, U. Krönert, G. Passler, J. Rikovska, and R. Wyss, Zeitschrift für Physik A Hadrons and Nuclei 342, 1 (1992), ISSN 0939-7922, URL http://dx. doi.org/10.1007/BF01294481.

[138] F. Le Blanc, D. Lunney, J. Obert, J. Oms, J. C. Putaux, B. Roussière, J. Sauvage, S. Zemlyanoi, J. Pinard, L. Cabaret, et al. (ISOLDE Collaboration), Phys. Rev. C 60, 054310 (1999), URL http://link.aps.org/doi/10.1103/PhysRevC.60.054310.

[139] J. Sauvage, N. Boos, L. Cabaret, J. Crawford, H. Duong, J. Genevey, M. Girod, G. Huber, F. Ibrahim, M. Krieg, et al., Hyperfine Interactions 129, 303 (2000), ISSN 0304-3843, URL http://dx.doi.org/10.1023/A\%3A1012618001695.

[140] G. Passler, J. Rikovska, E. Arnold, H. J. Kluge, L. Monz, R. Neugart, H. Ravn, and K. Wendt, Nuclear Physics A 580, 173 (1994), ISSN 0375-9474, URL http://www.sciencedirect.com/ science/article/pii/0375947494907692.

[141] F. Le Blanc, J. Obert, J. Oms, J. C. Putaux, B. Roussière, J. Sauvage, J. Pinard, L. Cabaret, H. T. Duong, G. Huber, et al. (and ISOLDE Collaboration), Phys. Rev. Lett. 79, 2213 (1997), URL http://link.aps.org/doi/10.1103/PhysRevLett.79.2213.

[142] A. E. Barzakh, L. K. Batist, D. V. Fedorov, V. S. Ivanov, K. A. Mezilev, P. L. Molkanov, F. V. Moroz, S. Y. Orlov, V. N. Panteleev, and Y. M. Volkov, Phys. Rev. C 88, 024315 (2013), URL http://link.aps.org/doi/10.1103/PhysRevC.88.024315.

[143] G. Ranjit, D. Kealhofer, G. D. Vukasin, and P. K. Majumder, Phys. Rev. A 89, 012511 (2014), URL http://link.aps.org/doi/10.1103/PhysRevA.89.012511.

[144] S. Dutta, R. Kirchner, O. Klepper, T. U. Kühl, D. Marx, G. D. Sprouse, R. Menges, U. Dinger, G. Huber, and S. Schröder, Zeitschrift für Physik A Hadrons and Nuclei 341, 39 (1991).

[145] A. Andreyev, K. Van de Vel, A. Barzakh, A. De Smet, H. De Witte, D. Fedorov, V. Fedoseyev, S. Franchoo, M. Górska, M. Huyse, et al., The European Physical Journal A - Hadrons and Nuclei 14, 63 (2002), ISSN 1434-6001, URL http://dx.doi.org/10.1140/epja/iepja1387.

[146] H. De Witte, A. N. Andreyev, N. Barré, M. Bender, T. E. Cocolios, S. Dean, D. Fedorov, V. N. Fedoseyev, L. M. Fraile, S. Franchoo, et al., Phys. Rev. Lett. 98, 112502 (2007), URL http: //link.aps.org/doi/10.1103/PhysRevLett.98.112502. 
[147] M. D. Seliverstov, A. N. Andreyev, N. Barré, A. E. Barzakh, S. Dean, H. De Witte, D. V. Fedorov, V. N. Fedoseyev, L. M. Fraile, S. Franchoo, et al., The European Physical Journal A 41, 315 (2009).

[148] P. Campbell, J. Behr, J. Billowes, G. Gwinner, G. Sprouse, and F. Xu, Nuclear Physics A 598, 61 (1996), ISSN 0375-9474, URL http://www.sciencedirect.com/science/article/ pii/0375947495004629.

[149] J. Kilgallon, M. Pearson, J. Billowes, P. Campbell, U. Georg, I. Grant, M. Keim, R. Neugart, M. Neuroth, and S. Wilbert, Physics Letters B 405, 31 (1997), ISSN 0370-2693, URL http: //www.sciencedirect.com/science/article/pii/S0370269397006060.

[150] M. R. Pearson, P. Campbell, K. Leerungnavarat, J. Billowes, I. S. Grant, M. Keim, J. Kilgallon, I. D. Moore, R. Neugart, M. Neurotz, et al., J. Phys. G: Nucl. Part. Phys. 26, 1829 (2000).

[151] T. E. Cocolios, W. Dexters, M. D. Seliverstov, A. N. Andreyev, S. Antalic, A. E. Barzakh, B. Bastin, J. Büscher, I. G. Darby, D. V. Fedorov, et al., Phys. Rev. Lett. 106, 052503 (2011), URL http://link.aps.org/doi/10.1103/PhysRevLett.106.052503.

[152] M. D. Seliverstov, T. E. Cocolios, W. Dexters, A. N. Andreyev, S. Antalic, A. E. Barzakh, B. Bastin, J. Büscher, I. G. Darby, D. V. Fedorov, et al., Physics Letters B 719, 362 (2013), ISSN 0370-2693, URL http://www.sciencedirect.com/science/article/pii/S0370269313000841.

[153] M. D. Seliverstov, T. E. Cocolios, W. Dexters, A. N. Andreyev, S. Antalic, A. E. Barzakh, B. Bastin, J. Büscher, I. G. Darby, D. V. Fedorov, et al., Phys. Rev. C 89, 034323 (2014), URL http://link.aps.org/doi/10.1103/PhysRevC.89.034323.

[154] D. A. Fink, T. E. Cocolios, A. N. Andreyev, S. Antalic, A. E. Barzakh, B. Bastin, D. V. Fedorov, V. N. Fedosseev, K. T. Flanagan, L. Ghys, et al., Phys. Rev. X 5, 011018 (2015), URL http: //link.aps.org/doi/10.1103/PhysRevX.5.011018.

[155] D. Kowalewska, K. Bekk, S. Göring, A. Hanser, W. Kälber, G. Meisel, and H. Rebel, Phys. Rev. A 44, 1442(R) (1991), URL http://link.aps.org/doi/10.1103/PhysRevA.44.R1442.

[156] U. Georg, M. Keim, A. Klein, R. Neugart, M. Neuroth, P. Lievens, R. Silverans, and L. Vermeeren, in Proceedings of the 1st International conference on exotic nuclei and atomic masses, ENAM1995 (Ed. Frontières, 1995).

[157] A. Voss, M. R. Pearson, J. Billowes, F. Buchinger, B. Cheal, J. E. Crawford, A. A. Kwiatkowski, C. D. P. Levy, and O. Shelbaya, Phys. Rev. Lett. 111, 122501 (2013), URL http://link.aps . org/doi/10.1103/PhysRevLett.111.122501.

[158] K. T. Flanagan, K. M. Lynch, J. Billowes, M. L. Bissell, I. Budinčević, T. E. Cocolios, R. P. de Groote, S. De Schepper, V. N. Fedosseev, S. Franchoo, et al., Phys. Rev. Lett. 111, 212501 (2013), URL http://link.aps.org/doi/10.1103/PhysRevLett.111.212501.

[159] K. M. Lynch, J. Billowes, M. L. Bissell, I. Budinčević, T. E. Cocolios, R. P. De Groote, S. De Schepper, V. N. Fedosseev, K. T. Flanagan, S. Franchoo, et al., Phys. Rev. X 4, 011055 (2014), URL http://link.aps.org/doi/10.1103/PhysRevX.4.011055.

[160] I. Budinčević, J. Billowes, M. L. Bissell, T. E. Cocolios, R. P. de Groote, S. De Schepper, V. N. Fedosseev, K. T. Flanagan, S. Franchoo, R. F. Garcia Ruiz, et al., Phys. Rev. C 90, 014317 (2014), URL http://link.aps.org/doi/10.1103/PhysRevC.90.014317. 
[161] R. Collister, G. Gwinner, M. Tandecki, J. A. Behr, M. R. Pearson, J. Zhang, L. A. Orozco, S. Aubin, and E. Gomez (FrPNC Collaboration), Phys. Rev. A 90, 052502 (2014), URL http: //link.aps.org/doi/10.1103/PhysRevA.90.052502.

[162] A. Voss, F. Buchinger, B. Cheal, J. E. Crawford, J. Dilling, M. Kortelainen, A. A. Kwiatkowski, A. Leary, C. D. P. Levy, F. Mooshammer, et al., Phys. Rev. C 91, 044307 (2015), URL http: //link.aps.org/doi/10.1103/PhysRevC.91.044307.

[163] J. S. Grossman, L. A. Orozco, M. R. Pearson, J. E. Simsarian, G. D. Sprouse, and W. Z. Zhao, Phys. Rev. Lett. 83, 935 (1999), URL http://link.aps.org/doi/10.1103/PhysRevLett.83. 935.

[164] J. M. Grossman, R. P. Fliller, T. E. Mehlstäubler, L. A. Orozco, M. R. Pearson, G. D. Sprouse, and W. Z. Zhao, Phys. Rev. A 62, 052507 (2000), URL http://link.aps.org/doi/10.1103/ PhysRevA.62.052507.

[165] E. Gomez, S. Aubin, L. A. Orozco, G. D. Sprouse, E. Iskrenova-Tchoukova, and M. S. Safronova, Phys. Rev. Lett. 100, 172502 (2008), URL http://link.aps.org/doi/10.1103/PhysRevLett. 100.172502.

[166] L. W. Wansbeek, S. Schlesser, B. K. Sahoo, A. E. L. Dieperink, C. J. G. Onderwater, and R. G. E. Timmermans, Phys. Rev. C 86, 015503 (2012), URL http://link.aps.org/doi/10. 1103/PhysRevC. 86.015503.

[167] C. J. Campbell, A. G. Radnaev, and A. Kuzmich, Phys. Rev. Lett. 106, 223001 (2011), URL http://link.aps.org/doi/10.1103/PhysRevLett.106.223001.

[168] V. Sonnenschein, I. D. Moore, S. Raeder, A. Hakimi, A. Popov, and K. Wendt, The European Physical Journal A 48, 1 (2012), ISSN 1434-6001, URL http://dx.doi.org/10.1140/epja/ i2012-12052-3.

[169] V. Sonnenschein, S. Raeder, A. Hakimi, I. D. Moore, and K. Wendt, Journal of Physics B: Atomic, Molecular and Optical Physics 45, 165005 (2012), URL http://stacks.iop.org/0953-4075/ $45 / i=16 / a=165005$.

[170] W. Kälber, J. Rink, K. Bekk, W. Faubel, S. Göring, G. Meisel, H. Rebel, and R. C. Thompson, Zeitschrift für Physik A Atomic Nuclei 334, 103 (1989), ISSN 0939-7922, URL http://dx.doi. org/10.1007/BF01294392.

[171] H. Schüler and H. Gollnow, Naturwissenschaften 22, 511 (1934), ISSN 0028-1042, URL http: //dx.doi.org/10.1007/BF01496571.

[172] Y. P. Gangrsky, S. G. Zemlyanoi, B. K. Kul'dzhanov, K. P. Marinova, and B. N. Markov, Bull. Acad. Sci. USSR (Phys. Ser.) 54, 13 (1990).

[173] A. Anatassov, Y. P. Gangrsky, K. P. Marinova, B. N. Markov, B. K. Kul'djanov, and S. G. Zemlyanoi, Hyperfine Interactions 74, 31 (1992), ISSN 0304-3843, URL http://dx.doi .org/10. 1007/BF02398614.

[174] S. Gerstenkorn, P. Luc, C. I. Bauche-Arnoult, and D. Merle, J. Phys. France 34, 805 (1973), URL http://dx.doi.org/10.1051/jphys:019730034010080500.

[175] M. Fred, F. S. Tomkins, J. E. Blaise, P. Camus, and J. Vergès, J. Opt. Soc. Am. 67, 7 (1977), URL http://www.opticsinfobase.org/abstract.cfm?URI=josa-67-1-7. 
[176] S. Gerstenkorn and F. Tomkins, Physica Scripta 36, 240 (1987), URL http://stacks.iop.org/ $1402-4896 / 36 / i=2 / a=009$.

[177] M. Fred and F. S. Tomkins, J. Opt. Soc. Am. 47, 1076 (1957), URL http://www. opticsinfobase.org/abstract.cfm?URI=josa-47-12-1076.

[178] C. E. Bemis, J. R. Beene, J. P. Young, and S. D. Kramer, Phys. Rev. Lett. 43, 1854 (1979), URL http://link.aps.org/doi/10.1103/PhysRevLett.43.1854.

[179] J. R. Beene, . J. Bemis, C. E., J. P. Young, and S. D. Kramer, Hyperfine Interactions 9, 143 (1981), ISSN 0304-3843, URL http://dx.doi.org/10.1007/BF01020910.

[180] M. W. Johnson, E. B. Shera, M. V. Hoehn, R. A. Naumann, J. D. Zumbro, and C. E. B. J. , Physics Letters B 161, 75 (1985), ISSN 0370-2693, URL http://www.sciencedirect.com/ science/article/pii/0370269385906124.

[181] B. L. Garrec and A. Petit, Journal of the Less Common Metals 122, 55 (1986), ISSN 0022-5088, proceedings of Actinides 85 - Part 2, URL http://www.sciencedirect.com/science/article/ pii/0022508886903929.

[182] H. Backe, R. R. Baum, B. Fricke, D. Habs, K. Hellmann, M. Hies, C. Illgner, C. Krameyer, H. Kunz, W. Lauth, et al., Hyperfine Interactions 97-98, 535 (1996), ISSN 0304-3843, URL http://dx.doi.org/10.1007/BF02150196.

[183] H. Backe, M. Hies, H. Kunz, W. Lauth, O. Curtze, P. Schwamb, M. Sewtz, W. Theobald, R. Zahn, K. Eberhardt, et al., Phys. Rev. Lett. 80, 920 (1998), URL http://link.aps.org/doi/10.1103/ PhysRevLett.80.920.

[184] H. Backe, A. Dretzke, D. Habs, M. Hies, G. Kube, H. Kunz, W. Lauth, H. J. Maier, R. Repnow, M. Sewtz, et al., Nuclear Physics A 690, 215 (2001), ISSN 0375-9474, URL http: //www.sciencedirect.com/science/article/pii/S0375947401009447.

[185] E. F. Worden, J. G. Conway, and J. Blaise, J. Opt. Soc. Am. B 3, 1092 (1986), URL http: //josab.osa.org/abstract.cfm?URI=josab-3-8-1092.

[186] E. F. Worden, J. Blaise, M. Fred, N. Trautmann, and J. F. Wyart, The Chemistry of the Actinide and Transactinide Elements, 9 (OSA, 2006).

[187] E. F. Worden, J. G. Conway, and J. Blaise, J. Opt. Soc. Am. B 4, 1358 (1987), URL http: //josab.osa.org/abstract.cfm?URI=josab-4-9-1358.

[188] J. G. Conway, E. F. Worden, and J. Blaise, J. Opt. Soc. Am. B 12, 1186 (1995), URL http: //josab.osa.org/abstract.cfm?URI=josab-12-7-1186.

[189] J.-F. Wyart, J. Blaise, and E. F. Worden, Journal of Solid State Chemistry 178, 589 (2005), ISSN 0022-4596, URL http://www. sciencedirect.com/science/article/pii/S0022459604005298.

[190] E. F. Worden, R. W. Lougheed, R. G. Gutmacher, and J. G. Conway, J. Opt. Soc. Am. 64, 77 (1974), URL http://www.opticsinfobase.org/abstract.cfm?URI=josa-64-1-77.

[191] H. Backe, A. Dretzke, S. Fritzsche, R. G. Haire, P. Kunz, W. Lauth, M. Sewtz, and N. Trautmann, Hyperfine Interactions 162, 3 (2005), ISSN 0304-3843, URL http://dx.doi.org/10. $1007 /$ s10751-005-9209-x. 
[192] G. Drake, ed., Springer handbook of Atomic, molecular and Optical physics (Springer, 2005).

[193] J. Bieroń and P. Pyykkö, Phys. Rev. Lett. 87, 133003 (2001), URL http://link.aps.org/doi/ 10.1103/PhysRevLett.87.133003.

[194] N. Stone, Nuclear Data Services, International Atomic Energy Agency (2011).

[195] N. Stone, Hyperfine Interactions pp. 1-10 (2015), ISSN 0304-3843, URL http://dx.doi.org/ 10.1007/s10751-014-1094-8.

[196] J. E. Rosenthal and G. Breit, Physical Review 41, 459 (1932), ISSN 0031-899X.

[197] A. Bohr and V. F. Weisskopf, Physical Review 77, 94 (1950), ISSN 0031-899X.

[198] W. King, Isotope Shifts in Atomic Spectra (New York: Plenum, 1984).

[199] B. Cheal, T. E. Cocolios, and S. Fritzsche, Phys. Rev. A 86, 042501 (2012), URL http://link. aps.org/doi/10.1103/PhysRevA.86.042501.

[200] G. Fricke, C. Bernhardt, K. Heilig, L. Schaller, L. Schellenberg, E. Shera, and C. Dejager, Atomic Data and Nuclear Data Tables 60, 177 (1995), ISSN 0092-640X, URL http://www . sciencedirect.com/science/article/pii/S0092640X85710078.

[201] E. C. SELTZER, Phys. Rev. 188, 1916 (1969), URL http://link.aps.org/doi/10.1103/ PhysRev.188.1916.

[202] J. Friedrich and N. Voegler, Nuclear Physics A 373, 192 (1982), ISSN 0375-9474, URL http: //www.sciencedirect.com/science/article/pii/0375947482901476.

[203] S. RAMAN, C. N. JR., and P. TIKKANEN, Atomic Data and Nuclear Data Tables 78, 1 (2001), ISSN 0092-640X, URL http://www.sciencedirect.com/science/article/pii/ S0092640X01908587.

[204] R. B. Cakirli, R. F. Casten, and K. Blaum, Phys. Rev. C 82, 061306 (2010), URL http://link. aps.org/doi/10.1103/PhysRevC.82.061306.

[205] I. Angeli and K. P. Marinova, Journal of Physics G: Nuclear and Particle Physics 42, 055108 (2015), URL http://stacks.iop.org/0954-3899/42/i=5/a=055108.

[206] J. Bonn, G. Huber, H.-J. Kluge, L. Kugler, and E. Otten, Physics Letters B 38, 308 (1972), ISSN 0370-2693, URL http://www.sciencedirect.com/science/article/pii/0370269372902535.

[207] T. W. Hänsch, Appl. Opt. 11, 895 (1972), URL http://ao.osa.org/abstract.cfm?URI= ao-11-4-895.

[208] U. Köpf, H. Besch, E. Otten, and C. von Platen, Zeitschrift für Physik 226, 297 (1969), ISSN 0044-3328, URL http://dx.doi.org/10.1007/BF01399103.

[209] G. Huber, C. Thibault, R. Klapisch, H. T. Duong, J. L. Vialle, J. Pinard, P. Juncar, and P. Jacquinot, Phys. Rev. Lett. 34, 1209 (1975), URL http://link.aps.org/doi/10.1103/ PhysRevLett.34.1209.

[210] B. Schinzler, W. Klempt, S. Kaufman, H. Lochmann, G. Moruzzi, R. Neugart, E.-W. Otten, J. Bonn, L. V. Reisky, K. Spath, et al., Physics Letters B 79, 209 (1978), ISSN 0370-2693, URL http://www.sciencedirect.com/science/article/pii/0370269378902241. 
[211] C. N. Davids, P. J. Woods, H. T. Penttilä, J. C. Batchelder, C. R. Bingham, D. J. Blumenthal, L. T. Brown, B. C. Busse, L. F. Conticchio, T. Davinson, et al., Phys. Rev. Lett. 76, 592 (1996), URL http://link.aps.org/doi/10.1103/PhysRevLett.76.592.

[212] R. Kirchner, Nuclear Instruments and Methods in Physics Research 186, 275 (1981), ISSN 0167-5087, URL http://www.sciencedirect.com/science/article/pii/0029554X81909162.

[213] R. Kirchner and E. Roeckl, Nuclear Instruments and Methods 133, 187 (1976), ISSN 0029-554X, URL http://www.sciencedirect.com/science/article/pii/0029554X76906078.

[214] V. N. Fedosseev, Y. Kudryavtsev, and V. I. Mishin, Physica Scripta 85, 058104 (2012), URL http://stacks.iop.org/1402-4896/85/i=5/a=058104.

[215] J. Ärje, J. Äystö, H. Hyvönen, P. Taskinen, V. Koponen, J. Honkanen, A. Hautojärvi, and K. Vierinen, Phys. Rev. Lett. 54, 99 (1985), URL http://link.aps.org/doi/10.1103/PhysRevLett. 54.99 .

[216] G. Savard, S. Baker, C. Davids, A. Levand, E. Moore, R. Pardo, R. Vondrasek, B. Zabransky, and G. Zinkann, Nuclear Instruments and Methods in Physics Research Section B: Beam Interactions with Materials and Atoms 266, 4086 (2008), ISSN 0168-583X, proceedings of the $\{$ XVth $\}$ International Conference on Electromagnetic Isotope Separators and Techniques Related to their Applications, URL http://www.sciencedirect.com/science/article/pii/S0168583X08006848.

[217] M. Wada, Y. Ishida, T. Nakamura, Y. Yamazaki, T. Kambara, H. Ohyama, Y. Kanai, T. M. Kojima, Y. Nakai, N. Ohshima, et al., Nuclear Instruments and Methods in Physics Research Section B: Beam Interactions with Materials and Atoms 204, 570 (2003), ISSN 0168-583X, 14th International Conference on Electromagnetic Isotope Separators and Techniques Related to their Applications, URL http://www.sciencedirect.com/science/article/pii/S0168583X02021511.

[218] T. Sonoda, M. Wada, H. Tomita, C. Sakamoto, T. Takatsuka, T. Furukawa, H. Iimura, Y. Ito, T. Kubo, Y. Matsuo, et al., Nuclear Instruments and Methods in Physics Research Section B: Beam Interactions with Materials and Atoms 295, 1 (2013), ISSN 0168-583X, URL http://www . sciencedirect.com/science/article/pii/S0168583X12006465.

[219] L. Vermeeren, N. Bijnens, M. Huyse, Y. A. Kudryavtsev, P. Van Duppen, J. Wauters, Z. N. Qamhieh, P. Thoen, E. Vandeweert, and R. E. Silverans, Phys. Rev. Lett. 73, 1935 (1994), URL http://link.aps.org/doi/10.1103/PhysRevLett.73.1935.

[220] Y. Kudryavtsev, J. Andrzejewski, N. Bijnens, S. Franchoo, J. Gentens, M. Huyse, A. Piechaczek, J. Szerypo, I. Reusen, P. V. Duppen, et al., Nuclear Instruments and Methods in Physics Research Section B: Beam Interactions with Materials and Atoms 114, 350 (1996), ISSN 0168-583X, URL http://www.sciencedirect.com/science/article/pii/0168583X96001942.

[221] K. Blaum, C. Geppert, H.-J. Kluge, M. Mukherjee, S. Schwarz, and K. Wendt, Nuclear Instruments and Methods in Physics Research Section B: Beam Interactions with Materials and Atoms 204, 331 (2003), ISSN 0168-583X, 14th International Conference on Electromagnetic Isotope Separators and Techniques Related to their Applications, URL http://www.sciencedirect. com/science/article/pii/S0168583X02019420.

[222] D. Fink, S. Richter, K. Blaum, R. Catherall, B. Crepieux, V. Fedosseev, A. Gottberg, T. Kron, B. Marsh, C. Mattolat, et al., Nuclear Instruments and Methods in Physics Research Section B: Beam Interactions with Materials and Atoms 344, 83 (2015), ISSN 0168-583X, URL http: //www.sciencedirect.com/science/article/pii/S0168583X14010222. 
[223] J. Ketelaer, J. Krämer, D. Beck, K. Blaum, M. Block, K. Eberhardt, G. Eitel, R. Ferrer, C. Geppert, S. George, et al., Nuclear Instruments and Methods in Physics Research Section A: Accelerators, Spectrometers, Detectors and Associated Equipment 594, 162 (2008), ISSN 0168-9002, URL http://www.sciencedirect.com/science/article/pii/S0168900208008693.

[224] H. Andra, Atomic Physics 4 (Plenum Press, New York, 1975).

[225] W. H. Wing, G. A. Ruff, W. E. Lamb, and J. J. Spezeski, Phys. Rev. Lett. 36, 1488 (1976), URL http://link.aps.org/doi/10.1103/PhysRevLett.36.1488.

[226] S. Kaufman, Optics Communications 17, 309 (1976), ISSN 0030-4018, URL http://www. sciencedirect.com/science/article/pii/0030401876902674.

[227] K. R. Anton, S. L. Kaufman, W. Klempt, G. Moruzzi, R. Neugart, E. W. Otten, and B. Schinzler, Phys. Rev. Lett. 40, 642 (1978), URL http://link.aps.org/doi/10.1103/PhysRevLett.40. 642.

[228] D. Eastham, A. Gilda, D. Warner, D. Evans, J. Griffiths, J. Billowes, M. Dancey, and I. Grant, Optics Communications 82, 23 (1991), ISSN 0030-4018, URL http://www.sciencedirect.com/ science/article/pii/003040189190184F.

[229] W. Klempt, J. Bonn, and R. Neugart, Physics Letters B 82, 47 (1979), ISSN 0370-2693, URL http://www.sciencedirect.com/science/article/pii/0370269379904222.

[230] R. V. Ambartsumyan, V. N. Kalinin, and V. S. Letokhov, JETP Lett. 13, 217 (1971).

[231] V. S. Letokhov, Laser Photoionization Spectroscopy (Orlando FL: Academic, 1987).

[232] G. S. Hurst and M. G. Payne, Principles and Applications of Resonance Ionzation Spectroscopy (Bristol: Hilger, 1988).

[233] P. V. Duppen, Nuclear Instruments and Methods in Physics Research Section B: Beam Interactions with Materials and Atoms 126, 66 (1997), international Conference on Electromagnetic Isotope Separators and Techniques Related to Their Applications, URL http://www . sciencedirect.com/science/article/pii/S0168583X96010178.

[234] Z.-T. Lu and K. D. A. Wendt, Review of Scientific Instruments 74, 1169 (2003), URL http: //scitation.aip.org/content/aip/journal/rsi/74/3/10.1063/1.1535232.

[235] G. D. Alkhazov, A. E. Barzakh, E. I. Berlovich, V. P. Denisov, A. G. Dernyatin, V. S. Ivanov, A. N. Zherikhin, O. N. Kompanets, V. S. Letokhov, V. I. Mishin, et al., JETP Lett. 37, 274 (1983).

[236] V. Fedoseyev, V. Letokhov, V. Mishin, G. Alkhazov, A. Barzakh, V. Denisov, A. Dernyatin, and V. Ivanov, Optics Communications 52, 24 (1984), ISSN 0030-4018, URL http://www. sciencedirect.com/science/article/pii/0030401884900671.

[237] V. I. Mishin, S. K. Sekatskii, V. N. Fedoseev, N. . B. Buyanov, V. S. Letokhov, G. D. Alkhazov, A. E. Barzakh, V. P. Denisov, V. S. Ivanov, and I. Y. Chubukov, Sov. Phys. JETP 66, 235 (1987).

[238] K. Wallmeroth, G. Bollen, A. Dohn, P. Egelhof, J. Grüner, F. Lindenlauf, U. Krönert, J. Campos, A. Rodriguez Yunta, M. J. G. Borge, et al., Phys. Rev. Lett. 58, 1516 (1987), URL http: //link.aps.org/doi/10.1103/PhysRevLett.58.1516. 
[239] U. Krönert, S. Becker, T. Hilberath, H.-J. Kluge, and C. Schulz, Applied Physics A 44, 339 (1987), ISSN 0947-8396, URL http://dx.doi.org/10.1007/BF00624601.

[240] J. K. P. Lee, G. Savard, J. E. Crawford, G. Thekkadath, H. T. Duong, J. Pinard, S. Liberman, F. Le Blanc, P. Kilcher, J. Obert, et al., AIP Conference Proceedings 164, 205 (1987), URL http://scitation.aip.org/content/aip/proceeding/aipcp/10.1063/1.37034.

[241] J. K. P. Lee, G. Savard, J. E. Crawford, G. Thekkadath, H. T. Duong, J. Pinard, S. Liberman, F. Le Blanc, P. Kilcher, J. Obert, et al., Phys. Rev. C 38, 2985 (1988), URL http://link. aps . org/doi/10.1103/PhysRevC.38.2985.

[242] H. Duong, J. Pinard, S. Liberman, G. Savard, J. Lee, J. Crawford, G. Thekkadath, F. L. Blanc, P. Kilcher, J. Obert, et al., Physics Letters B 217, 401 (1989), ISSN 0370-2693, URL http: //www.sciencedirect.com/science/article/pii/0370269389900683.

[243] A. Ezwam and J. Billowes, Hyperfine Interactions 162, 189 (2005), ISSN 0304-3843, URL http: //dx.doi.org/10.1007/s10751-005-9220-2.

[244] G. Alkhazov, A. Barzakh, V. Denisov, K. Mezilev, Y. Novikov, V. Panteleyev, A. Popov, E. Sudentas, V. Letokhov, V. Mishin, et al., Nuclear Instruments and Methods in Physics Research Section B: Beam Interactions with Materials and Atoms 69, 517 (1992), ISSN 0168-583X, URL http://www.sciencedirect.com/science/article/pii/0168583X9295309F.

[245] H. J. Kluge, F. Ames, W. Ruster, and K. Wallmeroth, in Proc. Acc. Radioact. Beams WS, TRIUMF Proceedings TRI-85-1, edited by L. Buchmann and J. M. D'Auria (1985), p. 119.

[246] S. Andreev, V. Mishin, and V. Letokhov, Optics Communications 57, 317 (1986), ISSN 00304018, URL http://www.sciencedirect.com/science/article/pii/003040188690266X.

[247] G. Alkhazov, E. Berlovich, and V. Panteleyev, Nuclear Instruments and Methods in Physics Research Section A: Accelerators, Spectrometers, Detectors and Associated Equipment 280, 141 (1989), ISSN 0168-9002, URL http://www.sciencedirect.com/science/article/pii/ 0168900289912825.

[248] V. Fedosseev, D. Fedorov, R. Horn, G. Huber, U. Köster, J. Lassen, V. Mishin, M. Seliverstov, L. Weissman, and K. Wendt, Nuclear Instruments and Methods in Physics Research Section B: Beam Interactions with Materials and Atoms 204, 353 (2003), ISSN 0168-583X, 14th International Conference on Electromagnetic Isotope Separators and Techniques Related to their Applications, URL http://www.sciencedirect.com/science/article/pii/S0168583X02019596.

[249] M. Seliverstov, A. Andreyev, N. Barré, H. Witte, D. Fedorov, V. Fedoseyev, S. Franchoo, J. Genevey, G. Huber, M. Huyse, et al., Hyperfine Interactions 171, 225 (2006), ISSN 0304-3843, URL http://dx.doi.org/10.1007/s10751-006-9486-z.

[250] V. Letokhov, Optics Communications 7, 59 (1973), ISSN 0030-4018, URL http://www. sciencedirect.com/science/article/pii/0030401873901119.

[251] K. L. Kratz, in Proc. Int. Conf. on Fission and Properties of Neutron-rich Nuclei, Sanibel Island, USA, edited by J. H. Hamilton and A. V. Ramayya (1998), p. 586.

[252] V. Fedoseyev, G. Huber, U. Köster, J. Lettry, V. Mishin, H. Ravn, and V. Sebastian, Hyperfine Interactions 127, 409 (2000), ISSN 0304-3843, URL http://dx.doi.org/10.1023/A\% 3A1012609515865. 
[253] S. Raeder, H. Heggen, J. Lassen, F. Ames, D. Bishop, P. Bricault, P. Kunz, A. Mj $\widetilde{A} \ddot{s}$, and A. TeigelhÃufer, Review of Scientific Instruments 85, 033309 (2014), URL http://scitation. aip.org/content/aip/journal/rsi/85/3/10.1063/1.4868496.

[254] Y. Kudriavtsev and V. Letokhov, Applied Physics B 29, 219 (1982), ISSN 0946-2171, URL http://dx.doi.org/10.1007/BF00688671.

[255] A. Nieminen, J. Huikari, A. Jokinen, J. Äystö, P. Campbell, and E. Cochrane, Nuclear Instruments and Methods in Physics Research Section A: Accelerators, Spectrometers, Detectors and Associated Equipment 469, 244 (2001), ISSN 0168-9002, URL http://www. sciencedirect. com/science/article/pii/S0168900200007506.

[256] K. T. Flanagan, Ph.D. thesis, University of Manchester, UK (2004).

[257] K. T. Flanagan, Acta Phys. Polonica B 44, 627 (2013).

[258] H. Franberg, P. Delahaye, J. Billowes, K. Blaum, R. Catherall, F. Duval, O. Gianfrancesco, T. Giles, A. Jokinen, M. Lindroos, et al., Nuclear Instruments and Methods in Physics Research Section B: Beam Interactions with Materials and Atoms 266, 4502 (2008), ISSN 0168-583X, proceedings of the $\{\mathrm{XVth}\}$ International Conference on Electromagnetic Isotope Separators and Techniques Related to their Applications, URL http://www.sciencedirect.com/science/article/ $\mathrm{pii} / \mathrm{S} 0168583 \mathrm{X} 08007647$.

[259] E. Mané, J. Billowes, K. Blaum, P. Campbell, B. Cheal, P. Delahaye, K. Flanagan, D. Forest, H. Franberg, C. Geppert, et al., The European Physical Journal A 42, 503 (2009), ISSN 1434-6001, URL http://dx.doi.org/10.1140/epja/i2009-10828-0.

[260] K. Lynch, T. Cocolios, and M. Rajabali, Hyperfine Interactions 216, 95 (2013), ISSN 0304-3843, URL http://dx.doi.org/10.1007/s10751-013-0820-y.

[261] M. Rajabali, K. Lynch, T. Cocolios, J. Billowes, M. Bissell, S. D. Schepper, K. Dewolf, K. Flanagan, F. L. Blanc, B. Marsh, et al., Nuclear Instruments and Methods in Physics Research Section A: Accelerators, Spectrometers, Detectors and Associated Equipment 707, 35 (2013), ISSN 01689002, URL http://www.sciencedirect.com/science/article/pii/S0168900212016464.

[262] J. A. Behr and G. Gwinner, Journal of Physics G: Nuclear and Particle Physics 36, 033101 (2009), URL http://stacks.iop.org/0954-3899/36/i=3/a=033101.

[263] S. Chu, Rev. Mod. Phys. 70, 685 (1998), URL http://link.aps.org/doi/10.1103/ RevModPhys.70.685.

[264] V. I. Balykin, V. G. Minogin, and V. S. Letokhov, Reports on Progress in Physics 63, 1429 (2000), URL http://stacks.iop.org/0034-4885/63/i=9/a=202.

[265] M. Tandecki, J. Zhang, R. Collister, S. Aubin, J. A. Behr, E. Gomez, G. Gwinner, L. A. Orozco, and M. R. Pearson, Journal of Instrumentation 8, P12006 (2013), URL http://stacks.iop. org $/ 1748-0221 / 8 / i=12 / a=P 12006$.

[266] J. Zhang, accepted for publication in Phys. Rev. Lett. (2015).

[267] W. Paul, Rev. Mod. Phys. 62, 531 (1990), URL http://link.aps.org/doi/10.1103/ RevModPhys.62.531. 
[268] F. Diedrich, E. Peik, J. M. Chen, W. Quint, and H. Walther, Phys. Rev. Lett. 59, 2931 (1987), URL http://link.aps.org/doi/10.1103/PhysRevLett.59.2931.

[269] H.-J. Kluge, T. Beier, K. Blaum, L. Dahl, S. Eliseev, F. Herfurth, B. Hofmann, O. Kester, S. Koszudowski, C. Kozhuharov, et al., in Current Trends in Atomic Physics, edited by S. Salomonson and E. Lindroth (Academic Press, 2008), vol. 53 of Advances in Quantum Chemistry, pp. 83 - 98, URL http://www.sciencedirect.com/science/article/pii/S0065327607530078.

[270] H. T. Schmidt, H. Cederquist, J. Jensen, and A. Fardi, Nuclear Instruments and Methods in Physics Research Section B: Beam Interactions with Materials and Atoms 173, 523 (2001), ISSN 0168-583X, URL http://www.sciencedirect.com/science/article/pii/ S0168583X00004158.

[271] P. Reinhed, A. Orban, S. Rosen, R. Thomas, I. Kashperka, H. Johansson, D. Misra, A. Fardi, L. BrÂd'nnholm, M. BjÂúrkhage, et al., Nuclear Instruments and Methods in Physics Research Section A: Accelerators, Spectrometers, Detectors and Associated Equipment 621, 83 (2010), ISSN 0168-9002, URL http://www.sciencedirect.com/science/article/pii/ S0168900210011988.

[272] J. Äystö, Nuclear Physics A 693, 477 (2001), ISSN 0375-9474, radioactive Nuclear Beams, URL http://www.sciencedirect.com/science/article/pii/S037594740100923X.

[273] G. Bollen, D. Morrissey, and S. Schwarz, Nuclear Instruments and Methods in Physics Research Section A: Accelerators, Spectrometers, Detectors and Associated Equipment 550, 27 (2005), ISSN 0168-9002, URL http://www.sciencedirect.com/science/article/pii/ S0168900205012301.

[274] S. S. Chouhan, G. Bollen, J. DeKamp, M. A. Green, D. Lawton, C. Magsig, D. J. Morrissey, J. Ottarson, S. Schwarz, and A. F. Zeller, Journal of Physics: Conference Series 507, 032010 (2014), URL http://stacks.iop.org/1742-6596/507/i=3/a=032010.

[275] P. Duppen, P. Dendooven, M. Huyse, L. Vermeeren, Z. Qamhieh, R. Silverans, and E. Vandeweert, Hyperfine Interactions 74, 193 (1992), ISSN 0304-3843, URL http://dx.doi.org/10. $1007 /$ BF02398629.

[276] Y. Kudryavtsev, B. Bruyneel, M. Huyse, J. Gentens, P. V. den Bergh, P. V. Duppen, and L. Vermeeren, Nuclear Instruments and Methods in Physics Research Section B: Beam Interactions with Materials and Atoms 179, 412 (2001), ISSN 0168-583X, URL http://www. sciencedirect.com/ science/article/pii/S0168583X01005754.

[277] T. Kessler, I. Moore, Y. Kudryavtsev, K. Peräjärvi, A. Popov, P. Ronkanen, T. Sonoda, B. Tordoff, K. Wendt, and J. Äystö, Nuclear Instruments and Methods in Physics Research Section B: Beam Interactions with Materials and Atoms 266, 681 (2008), ISSN 0168-583X, URL http://www . sciencedirect.com/science/article/pii/s0168583X07018290.

[278] Y. Kudryavtsev, T. Cocolios, J. Gentens, M. Huyse, O. Ivanov, D. Pauwels, T. Sonoda, P. V. den Bergh, and P. V. Duppen, Nuclear Instruments and Methods in Physics Research Section B: Beam Interactions with Materials and Atoms 267, 2908 (2009), ISSN 0168-583X, URL http: //www.sciencedirect.com/science/article/pii/S0168583X09006788.

[279] I. Moore, T. Kessler, J. Äystö, J. Billowes, P. Campbell, B. Cheal, B. Tordoff, M. Bissel, and G. Tungate, Hyperfine Interactions 171, 135 (2006), ISSN 0304-3843, URL http://dx.doi .org/ 10.1007/s10751-006-9494-z. 
[280] R. Ferrer, B. Bastin, D. Boilley, P. Creemers, P. Delahaye, E. Liénard, X. Fléchard, S. Franchoo, L. Ghys, M. Huyse, et al., Nuclear Instruments and Methods in Physics Research Section B: Beam Interactions with Materials and Atoms 317, 570 (2013), ISSN 0168-583X, URL http: //www.sciencedirect.com/science/article/pii/S0168583X13008239.

[281] V. Sonnenschein, Ph.D. thesis, The University of Jyväskylä (2015).

[282] H. Backe, K. Eberhardt, R. Feldmann, M. Hies, H. Kunz, W. Lauth, R. Martin, H. Schöpe, P. Schwamb, M. Sewtz, et al., Nuclear Instruments and Methods in Physics Research Section B: Beam Interactions with Materials and Atoms 126, 406 (1997), ISSN 0168-583X, international Conference on Electromagnetic Isotope Separators and Techniques Related to Their Applications, URL http://www.sciencedirect.com/science/article/pii/S0168583X9601035X.

[283] M. Sewtz, H. Backe, A. Dretzke, G. Kube, W. Lauth, P. Schwamb, K. Eberhardt, C. Grüning, P. Thörle, N. Trautmann, et al., Phys. Rev. Lett. 90, 163002 (2003), URL http://link.aps . org/doi/10.1103/PhysRevLett.90.163002.

[284] W. Lauth, H. Backe, M. Dahlinger, I. Klaft, P. Schwamb, G. Schwickert, N. Trautmann, and U. Othmer, Phys. Rev. Lett. 68, 1675 (1992), URL http://link.aps.org/doi/10.1103/ PhysRevLett.68.1675.

[285] H. Backe, P. Kunz, W. Lauth, A. Dretzke, R. Horn, T. Kolb, M. Laatiaoui, M. Sewtz, D. Ackermann, M. Block, et al., The European Physical Journal D 45, 99 (2007), ISSN 1434-6060, URL http://dx.doi.org/10.1140/epjd/e2007-00198-1.

[286] I. D. Moore, J. Billowes, P. Campbell, T. Eronen, C. Geppert, A. Jokinen, P. Karvonen, T. Kessler, B. Marsh, A. Nieminen, et al., AIP Conference Proceedings 831, 511 (2006), URL http:// scitation.aip.org/content/aip/proceeding/aipcp/10.1063/1.2200999.

[287] T. Sonoda, T. Cocolios, J. Gentens, M. Huyse, O. Ivanov, Y. Kudryavtsev, D. Pauwels, P. V. den Bergh, and P. V. Duppen, Nuclear Instruments and Methods in Physics Research Section B: Beam Interactions with Materials and Atoms 267, 2918 (2009), ISSN 0168-583X, URL http: //www.sciencedirect.com/science/article/pii/S0168583X09007812.

[288] R. Ferrer, V. Sonnenschein, B. Bastin, S. Franchoo, M. Huyse, Y. Kudryavtsev, T. Kron, N. Lecesne, I. Moore, B. Osmond, et al., Nuclear Instruments and Methods in Physics Research Section B: Beam Interactions with Materials and Atoms 291, 29 (2012), ISSN 0168-583X, URL http://www.sciencedirect.com/science/article/pii/S0168583X12005289.

[289] M. Reponen, I. Moore, I. Pohjalainen, T. Kessler, P. Karvonen, J. Kurpeta, B. Marsh, S. Piszczek, V. Sonnenschein, and J. Äystö, Nuclear Instruments and Methods in Physics Research Section A: Accelerators, Spectrometers, Detectors and Associated Equipment 635, 24 (2011), ISSN 01689002, URL http://www.sciencedirect.com/science/article/pii/S0168900211002117.

[290] Y. Kudryavtsev, R. Ferrer, M. Huyse, P. V. den Bergh, and P. V. Duppen, Nuclear Instruments and Methods in Physics Research Section B: Beam Interactions with Materials and Atoms 297, 7 (2013), ISSN 0168-583X, URL http://www.sciencedirect.com/science/article/pii/ S0168583X12007525.

[291] R. Ferrer, to be submitted (2015).

[292] J. Mackin, R. R. Dasari, C. H. Holbrow, J. T. Hutton, D. E. Murnick, M. Otteson, W. W. Quivers, G. Shimkaveg, and M. S. Feld, Phys. Rev. Lett. 66, 1681 (1991), URL http://link.aps.org/ doi/10.1103/PhysRevLett.66.1681. 
[293] F. Ibrahim, D. Verney, M. Lebois, B. Roussiere, S. Essabaa, S. Franchoo, S. Gales, D. G. Mueller, C. Lau, F. L. Blanc, et al., Nuclear Physics A 787, 110 (2007), ISSN 0375-9474, proceedings of the Ninth International Conference on Nucleus-Nucleus Collisions (NN2006), URL http://www . sciencedirect.com/science/article/pii/S0375947406010025.

[294] Argonne Tandem Linear Accelerator System, http://www.phy.anl.gov/atlas/, accessed: 201501-31.

[295] D. Rodriguez, K. Blaum, W. Nörtershäuser, M. Ahammed, A. Algora, G. Audi, J. ÄẪ̌ystö, D. Beck, M. Bender, J. Billowes, et al., The European Physical Journal Special Topics 183, 1 (2010), ISSN 1951-6355, URL http://dx.doi.org/10.1140/epjst/e2010-01231-2.

[296] V. Zagrebaev, S. Zemlyanoy, E. Kozulin, Y. Kudryavtsev, V. Fedosseev, R. Bark, Z. Janas, and H. Othman, Hyperfine Interactions 227, 181 (2014), ISSN 0304-3843, URL http://dx.doi.org/ 10.1007/s10751-014-1064-1.

[297] S. Schmidt, C. Geppert, and Z. Andelkovic, Hyperfine Interactions 227, 29 (2014), ISSN 03043843, URL http://dx.doi.org/10.1007/s10751-014-1048-1.

[298] M. Laatiaoui, H. Backe, M. Block, F.-P. Hessberger, P. Kunz, F. Lautenschläger, W. Lauth, M. Sewtz, and T. Walther, The European Physical Journal D 68, 1 (2014), ISSN 1434-6060, URL http://dx.doi.org/10.1140/epjd/e2014-40617-6.

[299] Riisager, K. and Butler, P. and Huyse, M. and Krücken, R., CERN pp. 2007-008 (2007).

[300] I. Moore, T. Eronen, D. Gorelov, J. Hakala, A. Jokinen, A. Kankainen, V. Kolhinen, J. Koponen, H. Penttilä, I. Pohjalainen, et al., Nuclear Instruments and Methods in Physics Research Section B: Beam Interactions with Materials and Atoms 317, Part B, 208 (2013), ISSN 0168-583X, $\{X V I t h\}$ International Conference on ElectroMagnetic Isotope Separators and Techniques Related to their Applications, December 2-7, 2012 at Matsue, Japan, URL http://www . sciencedirect. com/science/article/pii/S0168583X13007143.

[301] K. Minamisono, P. Mantica, A. Klose, S. Vinnikova, A. Schneider, B. Johnson, and B. Barquest, Nuclear Instruments and Methods in Physics Research Section A: Accelerators, Spectrometers, Detectors and Associated Equipment 709, 85 (2013), ISSN 0168-9002, URL http: //www.sciencedirect.com/science/article/pii/S0168900213001034.

[302] FRIB - Facility for Rare Isotope Beams, http://accelconf . web.cern.ch/accelconf/pac2013/ papers/fryba1.pdf, accessed: 2015-01-31.

[303] PNPI - Petersburg Nuclear Physics Institute, http://www.pnpi.spb.ru/, accessed: 2015-01-31.

[304] T. Kubo, Nuclear Instruments and Methods in Physics Research Section B: Beam Interactions with Materials and Atoms 204, 97 (2003), ISSN 0168-583X, 14th International Conference on Electromagnetic Isotope Separators and Techniques Related to their Applications, URL http: //www.sciencedirect.com/science/article/pii/S0168583X02018967.

[305] Y. Hirayama, Y. Watanabe, N. Imai, H. Ishiyama, S. Jeong, H. Miyatake, M. Oyaizu, S. Kimura, M. Mukai, Y. Kim, et al., Nuclear Instruments and Methods in Physics Research Section B: Beam Interactions with Materials and Atoms 353, 4 (2015), ISSN 0168-583X, URL http:// www.sciencedirect.com/science/article/pii/S0168583X15003365.

[306] RISP - Rare Isotope Science Project, http://risp.ibs.re.kr/orginfo/info_blds.do, accessed: 2015-07-07. 
[307] DESIR - Decay, Excitation and Storage of Radioactive Ions, http://www.cenbg.in2p3.fr/ desir/IMG/pdf/DESIR-Technical-Proposal-V090105.pdf, accessed: 2015-01-31.

[308] J. Dilling, R. Krücken, and G. Ball, Hyperfine Interactions 225, 1 (2014), ISSN 0304-3843, URL http://dx.doi.org/10.1007/s10751-013-0877-7.

[309] M. Lebois, D. Verney, F. Ibrahim, S. Essabaa, F. Azaiez, M. C. Mhamed, E. Cottereau, P. V. Cuong, M. Ferraton, K. Flanagan, et al., Phys. Rev. C 80, 044308 (2009), URL http://link. aps.org/doi/10.1103/PhysRevC.80.044308.

[310] S. Purushothaman, M. P. Reiter, E. Haettner, P. Dendooven, T. Dickel, H. Geissel, J. Ebert, C. Jesch, W. R. Plass, M. Ranjan, et al., EPL (Europhysics Letters) 104, 42001 (2013), URL http://stacks.iop.org/0295-5075/104/i=4/a=42001.

[311] W. Plass, T. Dickel, S. Purushothaman, P. Dendooven, H. Geissel, J. Ebert, E. Haettner, C. Jesch, M. Ranjan, M. Reiter, et al., Nuclear Instruments and Methods in Physics Research Section B: Beam Interactions with Materials and Atoms 317, Part B, 457 (2013), ISSN 0168-583X, \{XVIth\} International Conference on ElectroMagnetic Isotope Separators and Techniques Related to their Applications, at Matsue, Japan, URL http://www.sciencedirect.com/science/article/pii/ S0168583X13008823.

[312] W. Nörtershäuser, I. Moore, and C. Geppert, Hyperfine Interactions 227, 125 (2014), ISSN 03043843, URL http://dx.doi.org/10.1007/s10751-013-0996-1.

[313] M. Lochmann, R. Jöhren, C. Geppert, Z. Andelkovic, D. Anielski, B. Botermann, M. Bussmann, A. Dax, N. Frömmgen, M. Hammen, et al., Phys. Rev. A 90, 030501 (2014), URL http://link. aps.org/doi/10.1103/PhysRevA.90.030501.

[314] J. Ullmann, Z. Andelkovic, A. Dax, W. Geithner, C. Geppert, C. Gorges, M. Hammen, V. Hannen, S. Kaufmann, K. KÃúnig, et al., Journal of Physics B: Atomic, Molecular and Optical Physics 48, 144022 (2015), URL http://stacks.iop.org/0953-4075/48/i=14/a=144022.

[315] Resonance Ionization Laser Ion Sources, http://cds.cern.ch/record/1967371/files/ CERN-2013-007-p203.pdf?version=1, accessed: 2015-01-31.

[316] I. Moore, P. Dendooven, and J. Ärje, Hyperfine Interactions 223, 17 (2014), ISSN 0304-3843, URL http://dx.doi.org/10.1007/s10751-013-0871-0.

[317] R. Ringle, P. Schury, T. Sun, G. Bollen, D. Davies, J. Huikari, E. Kwan, D. J. Morrisey, A. Prinke, J. Savory, et al., International Journal of Mass Spectrometry 251, 300 (2006), ISSN 1387-3806, URL <GotoISI> : //WOS: 000237232100030.

[318] Panteleev, V. N., Barzakh, A. E., Fedorov, D. V., Menna, M., Moroz, F. V., and Volkov, Yu. M., Eur. Phys. J. A 26, 147 (2005), URL http://dx.doi.org/10.1140/epja/i2005-10147-6.

[319] M. Wakasugi, T. Ohnishi, S. Wang, Y. Miyashita, T. Adachi, T. Amagai, A. Enokizono, A. Enomoto, Y. Haraguchi, M. Hara, et al., Nuclear Instruments and Methods in Physics Research Section B: Beam Interactions with Materials and Atoms 317, Part B, 668 (2013), ISSN 0168-583X, \{XVIth\} International Conference on ElectroMagnetic Isotope Separators and Techniques Related to their Applications, December 2012 at Matsue, Japan, URL http: //www.sciencedirect.com/science/article/pii/S0168583X13004394. 
[320] X. Yang, T. Furukawa, T. Wakui, T. Fujita, K. Imamura, Y. Mitsuya, M. Hayasaka, Y. Ichikawa, Y. Ishibashi, H. Shirai, et al., Phys. Rev. A 90, 052516 (2014), URL http://link.aps.org/doi/ 10.1103/PhysRevA.90.052516.

[321] S. Rothe, A. N. Andreyev, S. Antalic, A. Borschevsky, L. Capponi, T. E. Cocolios, H. De Witte, E. Eliav, D. V. Fedorov, V. N. Fedosseev, et al., Nat Commun 4, 1835 (2013).

[322] P. Kunz, C. Andreoiu, P. Bricault, M. Dombsky, J. Lassen, A. Teigelhofer, H. Heggen, and F. Wong, Review of Scientific Instruments 85, 053305 (2014), URL http://scitation.aip. org/content/aip/journal/rsi/85/5/10.1063/1.4878718.

[323] Y. Kudryavtsev, M. Facina, M. Huyse, J. Gentens, P. V. den Bergh, and P. V. Duppen, Nuclear Instruments and Methods in Physics Research Section B: Beam Interactions with Materials and Atoms 204, 336 (2003), ISSN 0168-583X, 14th International Conference on Electromagnetic Isotope Separators and Techniques Related to their Applications, URL http: //www.sciencedirect.com/science/article/pii/S0168583X02019468.

[324] Y. Liu, Hyperfine Interactions 227, 85 (2014), ISSN 0304-3843, URL http://dx.doi.org/10. 1007/s10751-013-0965-8.

[325] A. Antognini, F. Nez, K. Schuhmann, F. D. Amaro, F. Biraben, J. M. R. Cardoso, D. S. Covita, A. Dax, S. Dhawan, M. Diepold, et al., Science 339, 417 (2013), http:// www.sciencemag.org/content/339/6118/417.full.pdf, URL http://www.sciencemag.org/ content/339/6118/417. abstract.

[326] R. Pohl, A. Antognini, F. Nez, F. D. Amaro, F. Biraben, J. M. R. Cardoso, D. S. Covita, A. Dax, S. Dhawan, L. M. P. Fernandes, et al., Nature 466, 213 (2010), ISSN 0028-0836, URL http://dx.doi.org/10.1038/nature09250.

[327] P. J. Mohr, B. N. Taylor, and D. B. Newell, Rev. Mod. Phys. 84, 1527 (2012), URL http: //link.aps.org/doi/10.1103/RevModPhys.84.1527.

[328] R. Pohl, R. Gilman, G. A. Miller, and K. Pachucki, Annual Review of Nuclear and Particle Science 63, 175 (2013), http://dx.doi.org/10.1146/annurev-nucl-102212-170627, URL http://dx.doi.org/10.1146/annurev-nucl-102212-170627.

[329] C. E. Carlson, Progress in Particle and Nuclear Physics 82, 59 (2015), ISSN 0146-6410, URL http://www.sciencedirect.com/science/article/pii/S0146641015000034.

[330] S. Karshenboim, D. McKeen, and M. Pospelov, aXiv:1401.6154 (2014).

[331] G. Lee, J. Arrington, and R. Hill, aXiv:1505.01498v1 (2015).

[332] J. Arrington and I. Sick, aXiv:1505.02680v1 (2015).

[333] A. Antognini, F. Biraben, J. M. Cardoso, D. S. Covita, A. Dax, L. M. Fernandes, A. L. Gouvea, T. Graf, T. W. HÃd'nsch, M. Hildebrandt, et al., Canadian Journal of Physics 89, 47 (2011), http://dx.doi.org/10.1139/P10-113, URL http://dx.doi.org/10.1139/P10-113.

[334] I. Tanihata, H. Savajols, and R. Kanungo, Progress in Particle and Nuclear Physics 68, 215 (2013), ISSN 0146-6410, URL http://www.sciencedirect.com/science/article/pii/ S0146641012001081. 
[335] I. Tanihata, H. Hamagaki, O. Hashimoto, Y. Shida, N. Yoshikawa, K. Sugimoto, O. Yamakawa, T. Kobayashi, and N. Takahashi, Phys. Rev. Lett. 55, 2676 (1985), URL http://link.aps.org/ doi/10.1103/PhysRevLett.55.2676.

[336] E. Arnold, J. Bonn, R. Gegenwart, W. Neu, R. Neugart, E.-W. Otten, G. Ulm, and K. Wendt, Physics Letters B 197, 311 (1987), ISSN 0370-2693, URL http://www.sciencedirect.com/ science/article/pii/037026938790390X.

[337] E. Arnold, J. Bonn, A. Klein, R. Neugart, M. Neuroth, E. Otten, P. Lievens, H. Reich, and W. Widdra, Physics Letters B 281, 16 (1992), ISSN 0370-2693, URL http://www. sciencedirect.com/science/article/pii/0370269392902667.

[338] W. Geithner, S. Kappertz, M. Keim, P. Lievens, R. Neugart, L. Vermeeren, S. Wilbert, V. N. Fedoseyev, U. Köster, V. I. Mishin, et al., Phys. Rev. Lett. 83, 3792 (1999), URL http://link. aps.org/doi/10.1103/PhysRevLett.83.3792.

[339] Z.-T. Lu, P. Mueller, G. W. F. Drake, W. Nörtershäuser, S. C. Pieper, and Z.-C. Yan, Rev. Mod. Phys. 85, 1383 (2013), URL http://link.aps.org/doi/10.1103/RevModPhys.85.1383.

[340] S. C. Pieper and R. B. Wiringa, Annual Review of Nuclear and Particle Science 51, 53 (2001), http://dx.doi.org/10.1146/annurev.nucl.51.101701.132506, URL http://dx.doi.org/ 10.1146/annurev.nucl.51.101701.132506.

[341] E. Epelbaum, H. Krebs, D. Lee, and U.-G. Mei $\boldsymbol{\sigma}$ ner, Phys. Rev. Lett. 104, 142501 (2010), URL http://link.aps.org/doi/10.1103/PhysRevLett.104.142501.

[342] C. Forssén, G. Hagen, M. Hjorth-Jensen, W. Nazarewicz, and J. Rotureau, Physica Scripta 2013, 014022 (2013), URL http://stacks .iop.org/1402-4896/2013/i=T152/a=014022.

[343] P. Navrátil and B. R. Barrett, Phys. Rev. C 57, 3119 (1998), URL http://link.aps.org/doi/ 10.1103/PhysRevC.57.3119.

[344] H. Feldmeier, T. Neff, R. Roth, and J. Schnack, Nuclear Physics A 632, 61 (1998), ISSN 03759474, URL http://www.sciencedirect.com/science/article/pii/S0375947497008051.

[345] R. Roth, T. Neff, and H. Feldmeier, Progress in Particle and Nuclear Physics 65, 50 (2010), ISSN 0146-6410, URL http://www.sciencedirect.com/science/article/pii/S0146641010000335.

[346] K. Varga, Y. Suzuki, and R. Lovas, Nuclear Physics A 571, 447 (1994), ISSN 0375-9474, URL http://www.sciencedirect.com/science/article/pii/0375947494902216.

[347] M. Brodeur, T. Brunner, C. Champagne, S. Ettenauer, M. J. Smith, A. Lapierre, R. Ringle, V. L. Ryjkov, S. Bacca, P. Delheij, et al., Phys. Rev. Lett. 108, 052504 (2012), URL http: //link.aps.org/doi/10.1103/PhysRevLett.108.052504.

[348] Z.-C. Yan and G. W. F. Drake, Phys. Rev. Lett. 91, 113004 (2003), URL http://link.aps.org/ doi/10.1103/PhysRevLett.91.113004.

[349] M. Smith, M. Brodeur, T. Brunner, S. Ettenauer, A. Lapierre, R. Ringle, V. L. Ryjkov, F. Ames, P. Bricault, G. W. F. Drake, et al., Phys. Rev. Lett. 101, 202501 (2008), URL http://link. aps.org/doi/10.1103/PhysRevLett.101.202501.

[350] D. Borremans, D. Balabanski, K. Blaum, W. Geithner, S. Gheysen, P. Himpe, M. Kowalska, J. Lassen, P. Lievens, S. Mallion, et al., PHYSICAL REVIEW C 72 (2005), ISSN 0556-2813. 
[351] R. Neugart, D. L. Balabanski, K. Blaum, D. Borremans, P. Himpe, M. Kowalska, P. Lievens, S. Mallion, G. Neyens, N. Vermeulen, et al., Phys. Rev. Lett. 101, 132502 (2008), URL http: //link.aps.org/doi/10.1103/PhysRevLett.101.132502.

[352] M. Puchalski, J. Komasa, and K. Pachucki, Phys. Rev. A 87, 030502 (2013), URL http://link. aps.org/doi/10.1103/PhysRevA.87.030502.

[353] O. Sorlin and M.-G. Porquet, Progress in Particle and Nuclear Physics 61, 602 (2008), ISSN 0146-6410, URL http://www. sciencedirect.com/science/article/pii/S0146641008000380.

[354] C. Thibault, R. Klapisch, C. Rigaud, A. M. Poskanzer, R. Prieels, L. Lessard, and W. Reisdorf, Phys. Rev. C 12, 644 (1975), URL http://link.aps.org/doi/10.1103/PhysRevC.12.644.

[355] C. Détraz, M. Langevin, D. Guillemaud, M. Epherre, G. Audi, C. Thibault, and F. Touchard, Nuclear Physics A 394, 378 (1983), ISSN 0375-9474, URL http://www.sciencedirect.com/ science/article/pii/0375947483901112.

[356] G. Huber, F. Touchard, S. Büttgenbach, C. Thibault, R. Klapisch, H. T. Duong, S. Liberman, J. Pinard, J. L. Vialle, P. Juncar, et al., Phys. Rev. C 18, 2342 (1978), URL http://link.aps . org/doi/10.1103/PhysRevC.18.2342.

[357] D. T. Yordanov, M. Kowalska, K. Blaum, M. De Rydt, K. T. Flanagan, P. Lievens, R. Neugart, G. Neyens, and H. H. Stroke, Phys. Rev. Lett. 99, 212501 (2007), URL http://link.aps.org/ doi/10.1103/PhysRevLett.99.212501.

[358] E. K. Warburton, J. A. Becker, and B. A. Brown, Phys. Rev. C 41, 1147 (1990), URL http: //link.aps.org/doi/10.1103/PhysRevC.41.1147.

[359] T. Otsuka, M. Honma, T. Mizusaki, N. Shimizu, and Y. Utsuno, Progress in Particle and Nuclear Physics 47, 319 (2001), ISSN 0146-6410, URL http://www.sciencedirect.com/science/ article/pii/S0146641001001570.

[360] I. Talmi, Nuclear Physics A 423, 189 (1984), ISSN 0375-9474, URL http: //www . sciencedirect. com/science/article/pii/0375947484905876.

[361] V. Somà, A. Cipollone, C. Barbieri, P. Navrátil, and T. Duguet, Phys. Rev. C 89, 061301 (2014), URL http://link.aps.org/doi/10.1103/PhysRevC.89.061301.

[362] F. Wienholtz, D. Beck, K. Blaum, C. Borgmann, M. Breitenfeldt, R. B. Cakirli, S. George, F. Herfurth, J. D. Holt, M. Kowalska, et al., Nature 498, 346 (2013), ISSN 0028-0836, URL http://dx.doi.org/10.1038/nature12226.

[363] D. Steppenbeck, S. Takeuchi, N. Aoi, P. Doornenbal, M. Matsushita, H. Wang, H. Baba, N. Fukuda, S. Go, M. Honma, et al., Nature 502, 207 (2013), ISSN 0028-0836, URL http: //dx.doi.org/10.1038/nature12522.

[364] W. H. King, Isotope Shifts in Atomic Spectra (Plenum Press, 1984).

[365] K. Minamisono, P. F. Mantica, T. J. Mertzimekis, A. D. Davies, M. Hass, J. Pereira, J. S. Pinter, W. F. Rogers, J. B. Stoker, B. E. Tomlin, et al., Phys. Rev. Lett. 96, 102501 (2006), URL http://link.aps.org/doi/10.1103/PhysRevLett.96.102501.

[366] C. Thibault, F. Touchard, S. Büttgenback, R. Klapisch, M. de Saint Simon, H. T. Duong, P. Jacquinot, P. Juncar, S. Liberman, P. Pillet, et al., Phys. Rev. C 23, 2720 (1981). 
[367] D. Eastham, P. Walker, J. Smith, J. Griffith, D. Evans, S. Wells, M. Fawcett, and I. Grant, Optics Communications 60, 293 (1986), ISSN 0030-4018, URL http://www.sciencedirect. com/science/article/pii/0030401886901537.

[368] D. A. Eastham, P. M. Walker, J. R. H. Smith, D. D. Warner, J. A. R. Griffith, D. E. Evans, S. A. Wells, M. J. Fawcett, and I. S. Grant, Phys. Rev. C 36, 1583 (1987), URL http://link.aps . org/doi/10.1103/PhysRevC.36.1583.

[369] P. Lievens, R. Silverans, L. Vermeeren, W. Borchers, W. Neu, R. Neugart, K. Wendt, F. Buchinger, and E. Arnold, Physics Letters B 256, 141 (1991), ISSN 0370-2693, URL http://www. sciencedirect.com/science/article/pii/037026939190664C.

[370] G. Audi, F. Kondev, M. Wang, B. Pfeiffer, X. Sun, J. Blachot, and M. MacCormick, Chinese Physics C 36, 1157 (2012), URL http://stacks.iop.org/1674-1137/36/i=12/a=001.

[371] V. Manea, D. Atanasov, D. Beck, K. Blaum, C. Borgmann, R. B. Cakirli, T. Eronen, S. George, F. Herfurth, A. Herlert, et al., Phys. Rev. C 88, 054322 (2013), URL http://link.aps.org/ doi/10.1103/PhysRevC.88.054322.

[372] D. Zawischa, Physics Letters B 155, 309 (1985), ISSN 0370-2693, URL http://www. sciencedirect.com/science/article/pii/037026938591576X.

[373] D. Zawischa, U. Regge, and R. Stapel, Physics Letters B 185, 299 (1987), ISSN 0370-2693, URL http://www.sciencedirect.com/science/article/pii/0370269387910033.

[374] P. M. Goddard, P. D. Stevenson, and A. Rios, Phys. Rev. Lett. 110, 032503 (2013), URL http: //link.aps.org/doi/10.1103/PhysRevLett.110.032503.

[375] E. Nadjakov, K. Marinova, and Y. Gangrsky, Atomic Data and Nuclear Data Tables 56, 133 (1994), ISSN 0092-640X, URL http://www.sciencedirect.com/science/article/pii/ S0092640X84710047.

[376] S. A. Ahmad, W. Klempt, R. Neugart, E. W. Otten, P. G. Reinhard, G. Ulm, and K. Wendt, Nuclear Physics A 483, 244 (1988), ISSN 0375-9474, URL http://www.sciencedirect.com/ science/article/pii/0375947488905349.

[377] K. Heyde and J. L. Wood, Rev. Mod. Phys. 83, 1467 (2011), URL http://link.aps.org/doi/ 10.1103/RevModPhys.83.1467.

[378] A. Andreyev et al., Nature 405, 430 (2000).

[379] G. Ulm, S. K. Bhattacherjee, P. Dabkiewicz, G. Huber, H. J. Kluge, T. Kühl, H. Lochmann, E. W. Otten, K. Wendt, S. A. Ahmad, et al., Zeitschrift für Physik A Atomic Nuclei 325, 247 (1986), ISSN 0939-7922, URL http://dx.doi.org/10.1007/BF01294605.

[380] I. Angeli and K. Marinova, Atomic Data and Nuclear Data Tables 99, 69 (2013), ISSN 0092-640X, URL http://www.sciencedirect.com/science/article/pii/S0092640X12000265.

[381] A. Coc, C. Thibault, F. Touchard, H. T. Duong, P. Juncar, S. Liberman, J. Pinard, J. Lermé, J. L. Vialle, S. Büttgenbach, et al., Physics Letters B 163, 66 (1985).

[382] V. A. Dzuba, W. R. Johnson, and M. S. Safronova, Phys. Rev. A 72, 022503 (2005), URL http://link.aps.org/doi/10.1103/PhysRevA.72.022503. 
[383] R. J. Hull and H. H. Stroke, Phys. Rev. 122, 1574 (1961), URL http://link.aps.org/doi/10. 1103/PhysRev.122.1574.

[384] M. Barboza-Flores, O. Redi, and H. Stroke, Zeitschrift für Physik A Atoms and Nuclei 321, 85 (1985), ISSN 0939-7922, URL http://dx.doi.org/10.1007/BF01411950.

[385] P. Campbell, J. Billowes, J. Behr, G. Gwinner, G. Sprouse, and F. Xu, Physics Letters B 346, 21 (1995), ISSN 0370-2693, URL http://www.sciencedirect.com/science/article/ pii/037026939500014C.

[386] P.-G. Reinhard and H. Flocard, Nuclear Physics A 584, 467 (1995), ISSN 0375-9474, URL http://www.sciencedirect.com/science/article/pii/037594749400770N.

[387] H. Iimura and F. Buchinger, Phys. Rev. C 78, 067301 (2008), URL http://link.aps.org/doi/ 10.1103/PhysRevC.78.067301.

[388] J. Uusitalo, J. Sarén, S. Juutinen, M. Leino, S. Eeckhaudt, T. Grahn, P. T. Greenlees, U. Jakobsson, P. Jones, R. Julin, et al., Phys. Rev. C 87, 064304 (2013), URL http://link.aps.org/ doi/10.1103/PhysRevC.87.064304.

[389] Z. Kalaninová, A. N. Andreyev, S. Antalic, F. P. Hessberger, D. Ackermann, B. Andel, M. C. Drummond, S. Hofmann, M. Huyse, B. Kindler, et al., Phys. Rev. C 87, 044335 (2013), URL http://link.aps.org/doi/10.1103/PhysRevC.87.044335.

[390] J. Rossnagel, S. Raeder, A. Hakimi, R. Ferrer, N. Trautmann, and K. Wendt, Phys. Rev. A 85, 012525 (2012), URL http://link.aps.org/doi/10.1103/PhysRevA.85.012525.

[391] K. Wendt, T. Gottwald, C. Mattolat, and S. Raeder, Hyperfine Interactions pp. 1-13 (2014), ISSN 0304-3843, URL http://dx.doi.org/10.1007/s10751-014-1041-8.

[392] N. Edelstein and D. G. Karraker, J. Chem. Phys. 62, 938 (1975).

[393] J. K. Tuli, Brookhaven National Laboratory, Brookhaven (2011).

[394] S. Raeder, private communication (2014).

[395] S. Fritzsche, The European Physical Journal D - Atomic, Molecular, Optical and Plasma Physics 33, 15 (2005), ISSN 1434-6060, URL http://dx.doi.org/10.1140/epjd/e2005-00013-1.

[396] A. Borschevsky, E. Eliav, M. J. Vilkas, Y. Ishikawa, and U. Kaldor, Phys. Rev. A 75, 042514 (2007), URL http://link.aps.org/doi/10.1103/PhysRevA.75.042514.

[397] A. Borschevsky, E. Eliav, M. J. Vilkas, Y. Ishikawa, and U. Kaldor, The European Physical Journal D 45, 115 (2007), ISSN 1434-6060, URL http://dx.doi.org/10.1140/epjd/ e2007-00130-9.

[398] M. Leino, H. Kankaanpää, R.-D. Herzberg, A. Chewter, F. Hessberger, Y. Le Coz, F. Becker, P. Butler, J. Cocks, O. Dorvaux, et al., The European Physical Journal A - Hadrons and Nuclei 6, 63 (1999), ISSN 1434-6001, URL http://dx.doi.org/10.1007/s100500050318.

[399] A. Eichhorn, M. Elbel, W. Kamke, R. Quad, and J. Bauche, Zeitschrift für Physik A Atoms and Nuclei 305, 39 (1982), ISSN 0939-7922, URL http://dx.doi.org/10.1007/BF01415076. 
[400] F. Marin, C. Fort, M. Prevedelli, M. Inguscio, G. Tino, and J. Bauche, Zeitschrift für Physik D Atoms, Molecules and Clusters 25, 191 (1993), ISSN 0178-7683, URL http://dx.doi.org/10. $1007 / \mathrm{BF} 01426879$.

[401] C. Brandau, C. Kozhuharov, Z. Harman, A. Müller, S. Schippers, Y. S. Kozhedub, D. Bernhardt, S. Böhm, J. Jacobi, E. W. Schmidt, et al., Phys. Rev. Lett. 100, 073201 (2008), URL http: //link.aps.org/doi/10.1103/PhysRevLett.100.073201.

[402] K. Blaum, S. Nagy, and G. Werth, Journal of Physics B: Atomic, Molecular and Optical Physics 42, 154015 (2009), URL http://stacks.iop.org/0953-4075/42/i=15/a=154015.

[403] R. Wolf, F. Wienholtz, D. Atanasov, D. Beck, K. Blaum, C. Borgmann, F. Herfurth, M. Kowalska, S. Kreim, Y. A. Litvinov, et al., International Journal of Mass Spectrometry 349âĂŞ350, 123 (2013), ISSN 1387-3806, 100 years of Mass Spectrometry, URL http://www.sciencedirect. $\mathrm{com} / \mathrm{science/article/pii/S1387380613001115.}$ 